Published in final edited form as:

Tetrahedron. 2007 June 25; 63(26): 5768-5796.

\title{
Total Synthesis of Amphidinolide E and Amphidinolide E Stereoisomers
}

\author{
Porino Va and William R. Roush ${ }^{*}$ \\ Departments of Chemistry and Biochemistry, Scripps Florida, Jupiter, Florida 33458
}

\section{Abstract}

Four amphidinolide E stereoisomers, amphidinolide E (1), 2-epi-amphidinolide E (2), 19-epiamphidinolide $\mathrm{E}(\mathbf{3})$, and 2-epi-19-epi-amphidinolide $\mathrm{E}(\mathbf{4})$, have been synthesized via the judicious union of aldehyde $\mathbf{5}$, allylsilanes $\mathbf{7}$ or $\mathbf{8}$, acids $\mathbf{9}$ or $\mathbf{1 0}$, and vinylstannane $\mathbf{6}$. The C19 stereocenters of the $\mathrm{C} 19$ epimeric allylsilanes $\mathbf{7}$ and $\mathbf{8}$ were introduced via crotylboration reactions early in the synthesis. [3+2]-Annulation reactions of aldehyde 5 with allylsilanes $\mathbf{7}$ and $\mathbf{8}$ were employed to set the core tetrahydrofuran units of $\mathbf{1 - 4}$. Finally, the $\mathrm{C} 2$ stereocenter was installed by esterification using acid $\mathbf{9}$, without incident, or with acid 10, in which case an unexpected and completely stereoselective inversion of $\mathrm{C} 2$ occurs.

\section{Keywords}

amphidinolide E stereoisomers; [3+2] annulation reaction; esterification of $\mathrm{Fe}(\mathrm{CO})_{3}$-complexed dienoic acid

\section{Introduction}

The amphidinolides are a family of biologically active macrolides isolated from the dinoflagellate Amphidinium sp. ${ }^{1}$ Many of the amphidinolides possess striking cytotoxic properties. Furthermore, this family of natural products exhibits a high degree of structural diversity despite being isolated from a common source. As a consequence, the amphidinolides have attracted considerable interest as targets for synthesis and biological evaluation. Total syntheses of amphidinolides $\mathrm{A},{ }^{2} \mathrm{~J},{ }^{3} \mathrm{~K},{ }^{4} \mathrm{P},{ }^{5} \mathrm{~T},{ }^{6} \mathrm{~W},{ }^{7} \mathrm{X}^{8}$ and $\mathrm{Y}^{9}$ have been reported.

Amphidinolide $\mathrm{E}^{10}(\mathbf{1})$ is a 19 -membered biologically active ${ }^{1 \mathrm{c}}$ macrolactone featuring an embedded 2,5-cis-tetrahydrofuran (Figure 1). This structural motif is common within the amphidinolide family. However, the $\mathrm{C}(1)$-C(6) $\alpha$-chiral, $\beta, \gamma, \delta, \varepsilon$-dienoate moiety is unique to amphidinolide E. Lee has recently reported the total synthesis of amphidinolide $\mathrm{E}, 11$ while Gurjar $^{12}$ and Marshall ${ }^{13}$ have published studies toward the synthesis of this interesting natural product.

As part of a program directed towards the synthesis of tetrahydrofuran-containing natural products ${ }^{14}$ using a [3+2] annulation strategy, 15,16 we developed and reported a convergent and stereoselective total synthesis of amphidinolide E. ${ }^{17}$ In the course of these studies, we encountered an unexpected and highly selective $\mathrm{C} 2$ inversion during an esterification reaction

\footnotetext{
*Corresponding author: Email: roush@ @scripps.edu, Office Phone Number: (561) 799-8880, Fax Number: (561) 799-8955.

Publisher's Disclaimer: This is a PDF file of an unedited manuscript that has been accepted for publication. As a service to our customers we are providing this early version of the manuscript. The manuscript will undergo copyediting, typesetting, and review of the resulting proof before it is published in its final citable form. Please note that during the production process errors may be discovered which could affect the content, and all legal disclaimers that apply to the journal pertain.
} 
$(\mathbf{2 5}+\mathbf{1 0} \rightarrow \mathbf{3 9})$ that ultimately led to the inadvertent synthesis of 2-epi-amphidinolide E (2). 18 Initially, we were unaware of this $\mathrm{C} 2$ inversion and were faced with the conundrum as to why 2-epi-amphidinolide E (2), which at the time we thought was structure $\mathbf{1}$, did not have spectroscopic properties that matched the data reported in the literature for amphidinolide $\mathrm{E}$ (1). ${ }^{10} \mathrm{We}$ describe herein the various structural correlation experiments undertaken to unravel this problem, which ultimately led to the syntheses of amphidinolide $\mathrm{E}(\mathbf{1})$, 2-epi-amphidinolide E (2), 19-epi-amphidinolide E (3), and 2-epi-19-epi-amphidinolide E (4).

\section{Results and discussion}

\subsection{Synthesis of 2-epi-amphidinolide $E$}

We envisioned that amphidinolide E could be obtained by the Stille ${ }^{19}$ cross coupling of vinyl iodide 11 with vinylstannane $\mathbf{6}$ (Figure 1). Macrocycle 11 would be accessed in two steps via esterification of $\mathbf{1 2}$ with dienoic acid $\mathbf{1 3}$, followed by ring closing metathesis. Finally, we anticipated that the tetrahydrofuran fragment 12 would arise from the product of the $[3+2]$ annulation of aldehyde 5 and allylsilane $7 .^{14 a}, 15$

The synthesis of aldehyde 5 began with the Swern oxidation of alcohol 14, ${ }^{20}$ which is available in five steps from commercially available isopropylidene dimethyl D-tartrate (Scheme 1). Treatment of the aldehyde with vinyl magnesium bromide followed by a Johnson orthoester Claisen rearrangement ${ }^{21}$ of the allylic alcohol intermediate afforded methyl ester 15 in $60 \%$ overall yield. Reduction of $\mathbf{1 5}$ with DIBAL $\left(-78^{\circ} \mathrm{C}\right)$ yielded the targeted aldehyde $\mathbf{5}$.

Allylsilane 7 was synthesized starting from homoallylic alcohol 16, ${ }^{22}$ which is available with high diastereoselectivity from the asymmetric $(E)$-crotylboration ${ }^{23}$ of L-glyceraldehyde pentylidene ketal $^{24}$ (Scheme 2). Protection of $\mathbf{1 6}$ as the $p$-methoxybenzyl ether followed by hydroboration-oxidation of the vinyl group provided primary alcohol 17 (90\% yield). Oxidation of 17, by using $\mathrm{SO}_{3}$.pyridine and DMSO, ${ }^{25}$ and subsequent Corey-Fuchs ${ }^{26}$ homologation of the aldehyde furnished alkyne $18(88 \%)$. Acidic hydrolysis of the pentylidene ketal protecting group and oxidative cleavage of the resulting diol afforded aldehyde 19. antiSilylallylboration of $\mathbf{1 9}$ was accomplished with 9:1 selectivity (90\% yield) by using $(E)-\gamma$ silylallylboronate $(S, S)-\mathbf{2 0} .^{27}$ Protection of the $\beta$-hydroxy allylsilane $\mathbf{2 1}$ as the triethylsilyl ether provided allylsilane coupling partner 7. Mosher ester analysis of alcohol 21 confirmed the $\mathrm{C} 17(S)$ hydroxyl stereocenter (Scheme 3). ${ }^{28}$ In addition, the $\mathrm{C} 16-\mathrm{C} 17$ relative stereochemistry was confirmed via basic Peterson elimination ${ }^{29}$ of 21, which afforded the $Z$ diene 23.

Initial [3+2] annulations using excess aldehyde $\mathbf{5}$, with respect to allylsilane $\mathbf{7}$, and substiochiometric amounts of $\mathrm{BF}_{3} \cdot \mathrm{Et}_{2} \mathrm{O}$ afforded low yields of product $\mathbf{2 4}$ (entry 1, Scheme 4). On the other hand, use of stoichiometric or excess amounts of allylsilane 7 and stoichiometric amounts of Lewis acid led to improved yields of 24 (entries 3-5). The optimum reaction stiochiometry, 2.5 equiv of $\mathbf{7}$ and 1 equiv of $\mathbf{5}$, led to $\mathbf{2 4}$ in $48 \%$ yield and d.r. $>20: 1$. Use of $\mathrm{SnCl}_{4}$ as the Lewis acid led to trace amounts of $\mathbf{2 4}$ and significant decomposition of 7 (entry 2). ${ }^{30}$ Excess allylsilane 7 was recovered in excellent yield for all reactions using $\mathrm{BF}_{3} \cdot \mathrm{Et}_{2} \mathrm{O}$. The modest yield of $\mathbf{2 4}$ is due to the propensity of $\mathbf{5}$ to cyclotrimerize under the reaction conditions to give 26. Slow syringe pump addition of $\mathbf{5}$ into a $-78{ }^{\circ} \mathrm{C}$ solution of allylsilane 7 and $\mathrm{BF}_{3} \cdot \mathrm{Et}_{2} \mathrm{O}$ failed to improve the yield. In addition, conducting the reaction at temperatures higher than $-78{ }^{\circ} \mathrm{C}$ resulted in significant Peterson elimination of 7 .

Treatment of [3+2] adduct $\mathbf{2 4}$ with solid TBAF $3 \mathrm{H}_{2} \mathrm{O}$ in DMF at $90{ }^{\circ} \mathrm{C}$ effected smooth sp 3 $\mathrm{C}-\mathrm{Si}$ bond scission with concomitant removal of the triethylsilyl ether (Scheme 4). ${ }^{31}$ Reintroduction of the TES ether, and subsequent oxidative removal of the $p$-methoxybenzyl 


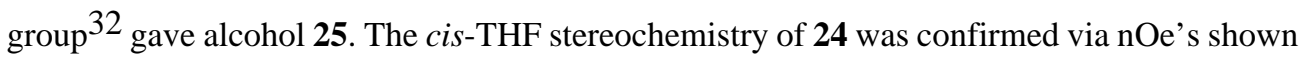
Figure 2.

Esterification of the C18 hydroxyl group of $\mathbf{2 5}$ (or related intermediates 27, 28, and 29) with dienoic acid $\mathbf{1 3}^{33}$ (or various derivatives of $\mathbf{1 3}$ ) proved to be extremely challenging (Table 1). Use of excess amounts (10-20 equiv.) of $\mathbf{1 3}$ and various coupling reagents invariably failed. The list of unsuccessful esterification reactions included attempts to use the modified Yamaguchi conditions ${ }^{34}$ (entry 1), use of mild peptide coupling conditions ${ }^{35}$ (entries 2, 3 and 6 ), use of Otera's transesterification catalyst $3^{36}$ (entry 5), attempted coupling of the tributyltin ether of $\mathbf{2 9}$ with the acylfluoride $\mathbf{3 2}$ (entry 7), and generation of the lithium alkoxide of 29 followed by treatment with acylfluoride 32 (entry 8). Kita ${ }^{37}$ has developed a two step esterification protocol involving initial formation of a 1-ethoxyvinyl ester derivative of the acid coupling partner using 1-ethoxyacetylene and $\left\{\mathrm{RuCl}_{2} \text { (p-cymene) }\right\}_{2}$. This 1-ethoxyvinyl ester derivative is then treated with the alcohol coupling partner and a catalytic amount of a Bronsted acid to achieve the esterification. Lee and co-workers ${ }^{11}$ have successfully employed this methodology in a macrolactonization fashion for amphidinolide E. Unfortunately, the Kita conditions proved to be unsuccessful in our intermolecular reaction (entry 9). Whereas in most cases the alcohol was recovered unscathed from these unsuccessful experiments, the acid component was recovered as the fully conjugated, diene migrated species $\mathbf{3 4}$. No more than trace quantities of ester products corresponding to acids $\mathbf{1 3}$ or $\mathbf{3 4}$ could be isolated from these experiments.

We reasoned that use of a "diene protected" acid $\mathbf{1 0}$ might be effective to avoid the problems encountered in attempted esterifications reactions of acid 13. The synthesis of acid $\mathbf{1 0}$ began with the Evans methylation ${ }^{38}$ of oxazolidinone $\mathbf{3 5}^{39}$ to afford product 36 in $79 \%$ yield (Scheme 5). Treatment of $\mathbf{3 6}$ with $\mathrm{Fe}_{2}(\mathrm{CO})_{9}$ in benzene at reflux gave a separable 1:1 mixture of $\mathbf{3 7}$ and $\mathbf{3 8}$. Hydrolysis of the acyloxazolidinone units of $\mathbf{3 7}$ and $\mathbf{3 8}$ furnished acids $\mathbf{1 0}$ and $\mathbf{9}^{40}$ in $62 \%$ and $58 \%$ yield, respectively.

Gratifyingly, use of $(\mathrm{CO})_{3} \mathrm{Fe}$-complexed dienoic acid $\mathbf{1 0}$ (1.6 equiv) resulted in an efficient esterification with alcohol 25 under the modified Yamaguchi conditions (Scheme 6). However, the ester product 39 is the unexpected, C2 inverted isomer. Since 39 was formed as a single diastereomer, we had no reason to suspect inversion at $C 2$ and therefore proceeded forward with the synthesis under the assumption that the $2 S$ stereochemistry of $\mathbf{1 0}$ had been preserved after the esterification reaction. It was not until much later (see section 2.4) that we became aware of the C2 inversion in this reaction.

Oxidative decomplexation of the $(\mathrm{CO})_{3} \mathrm{Fe}-$ unit of $\mathbf{3 9}$ (96\% yield) followed by ring closing metathesis 41,42 (60\% yield) afforded the 19-membered macrocycle 40. Furthermore, an inseparable mixture of products thought to arrive by enyne metathesis was also isolated (15\% yield). Use of the more active Grubbs' second generation or Grubbs-Hoveyda catalysts resulted in significant decomposition of the polyene substrate. Diene and triene forming ring closing metathesis macrocyclizations can sometimes be plague with products containing rings smaller than desired. ${ }^{42 a, b}$ However, none of the smaller macrocycles (16-membered ring and smaller) were observed for the ring closure our polyene substrate. In addition, ruthenium catalyzed ring closing metathesis reactions of substrates containing internal alkynes, ${ }^{43}$ unprotected terminal alkynes, ${ }^{44}$ and protected terminal alkynes (silylated 45 or dicobalt complexed ${ }^{46}$ ) are rare.

Stannylalumination-protonolysis ${ }^{47}$ of the alkyne unit of $\mathbf{4 0}$ followed by iododestannylation of the resultant vinylstannane gave vinyl iodide $\mathbf{4 1}$. Acidic hydrolysis of both the triethylsilyl and acetonide protecting groups afforded a 10:1 inseparable mixture of the $\mathrm{C} 18$ and $\mathrm{C} 17$ lactones. Stille cross coupling of the mixture of lactonic iodides with vinylstannane $\mathbf{6}^{12}$ followed by HPLC purification afforded 2-epi-amphidinolide E (2), spectroscopic data for which did not 
match Kobayashi's spectroscopic data for amphidinolide E (1). The most egregious spectroscopic disagreement between $\mathbf{2}$ and natural amphidinolide E (1) was the chemical shift for the $\mathrm{H} 3$ proton $(6.00 \mathrm{ppm}$ for $\mathbf{2}$ vs. $5.59 \mathrm{ppm}$ for natural $\mathbf{1})$.

\subsection{Structural correlations of our intermediates with Kobayashi's amphidinolide E degradation products}

In an effort to determine the structural discrepancies between natural amphidinolide $\mathrm{E}(\mathbf{1})$ and what we believed was our "synthetic amphidinolide E" (2), we proceeded to repeat Kobayashi's stereochemical assignments $10 \mathrm{~b}$ for $\mathbf{1}$ using our synthetic intermediates as correlation compounds.

Kobayashi and co-workers transformed natural amphidinolide E (1) into two degradation products, the $\mathrm{C} 8-\mathrm{C} 17$ tetrahydrofuran containing fragment $\mathbf{4 2}$ and the $\mathrm{C} 1-\mathrm{C} 7$ fragment $\mathbf{4 3}$ (Scheme 7). ${ }^{10}$ The enantiomer of $\mathbf{4 2}$, namely compound $\mathbf{4 4}$, was independently synthesized by Kobayashi, thereby leading to the assignment of $13 S$ and $16 S$ stereochemistry in natural amphidinolide $\mathrm{E}$. The $2 R$ stereochemistry of natural amphidinolide $\mathrm{E}$ was assigned by analogy to data for a small set of structures containing primary Mosher esters with adjacent methylbranched stereocenters. ${ }^{48}$ This precedent indicated that the difference in chemical shifts for the diastereotopic $\mathrm{C} 1$ methylene protons were typically smaller for $(S)$-MTPA esters when the adjacent methyl-branched stereocenter has $R$ stereochemistry. We were concerned about the reliability of this method for absolute stereochemical assignment, given the small number of literature examples, and therefore resolved to make an unequivocal stereochemical assignment for $\mathrm{C} 2$ by independently synthesizing the $\mathrm{C} 1-\mathrm{C} 7$ fragment $\mathbf{4 3}$ from a chiral pool starting material, aldehyde $\mathbf{4 5}^{49}$ (Scheme 8). Olefination of aldehyde $\mathbf{4 5}$ afforded product $\mathbf{4 6} .50$ Hydrogenation of $\mathbf{4 6}$ in EtOAc-MeOH over Pd/C occurred with concomitant hydrolysis of the primary TBS ether. Oxidative removal of the PMB ether of $\mathbf{4 7}$ followed by Mosher ester formation yielded the $\mathrm{C} 1-\mathrm{C} 7$ fragment $\mathbf{4 3}$. The ${ }^{1} \mathrm{H}$ NMR data for our synthetic $\mathbf{4 3}$ matched Kobayashi's data exactly. Therefore $\mathrm{C} 2$ of $\mathbf{4 3}$, and hence also of amphidinolide $\mathrm{E}$, is $R$.

Kobayashi and co-workers treated natural amphidinolide E with 2,2-dimethoxypropane and $p$-toluenesulfonic acid to generate the C7-C8 acetonide derivative 49 (Scheme 9). ${ }^{10}$ The magnitude of the H7-H8 coupling constant and the indicated NOESY correlation peaks of 49 formed the basis for assignment of threo $\mathrm{C} 7-\mathrm{C} 8$ relative stereochemistry. The $\mathrm{C} 7$ and $\mathrm{C} 8$ absolute stereochemistry was assigned by application of the exciton chirality method ${ }^{51}$ for the 7,8-bis-cinnamoyl ester derivative $\mathbf{5 0}$. The $\mathrm{CD}$ spectrum of $\mathbf{5 0}$ showed a negative first Cotton effect ( $\lambda_{\text {ext }} 324 \mathrm{~nm}, \Delta \varepsilon-14.3$ ) and positive second Cotton effect $\left(\lambda_{\text {ext }} 289 \mathrm{~nm}, \Delta \varepsilon+18.9\right)$, indicating $7 R$ and $8 R$ absolute stereochemistry. 52

Kobayashi's NMR analysis of the 7,8,17-tris-MTPA ester derivative $\mathbf{5 1}$ confirmed the $7 R$ and $8 R$ assignments and determined the $17 R$ stereocenter of natural amphidinolide $\mathrm{E}$ (Scheme 10a).

10 Deprotection of our ring closing metathesis product 40, followed by Mosher ester formation afforded the synthetic correlation Mosher triester 52 (Scheme 10b). Mosher triester 52 lacks the complete side chain of Kobayashi's intermediate 51. Therefore, the magnitudes of the chemical shift differences for the $(S)$ versus $(R)$-MTPA ester derivatives were not expected to be identical. However, if the stereocenters in $\mathbf{5 1}$ and $\mathbf{5 2}$ were the same, we expected the directionalities of the chemical shift differences $\left(\Delta \delta=\delta_{S}-\delta_{R}\right)$ to correlate. This held true at every position except for $\mathrm{C} 17$ and $\mathrm{C} 3$. We thought this discrepancy was an indication that we had incorrectly assigned the C16 stereochemistry of $\mathbf{5 2}$, and ultimately $\mathbf{4 0}$ and $\mathbf{2 5}$, as $16 S$ and that perhaps the correct stereochemistry is $16 R$. Therefore, our intermediate $\mathbf{2 5}$ was transformed into Kobayashi's C8-C17 fragment 42 in eight standard steps (Scheme 11). The ${ }^{1} \mathrm{H}$ NMR data for our synthetic $\mathbf{4 2}$ matched Kobayashi's data, thereby confirming that our original $16 \mathrm{~S}$ stereochemical assignment was correct. 
Kobayashi and co-workers assigned the $\mathrm{C} 18$ and $\mathrm{C} 19$ stereocenters by synthesizing the bisacetonide intermediate $\mathbf{5 5}$ from natural amphidinolide $\mathrm{E}$ in three steps (Scheme 12). ${ }^{10}$ The NOESY correlation peaks of 55 established a threo C17-C18 relationship. In addition, the H18H19 and H18-C28 coupling constants of 1 supported a erythro C18-C19 relationship. The red NOESY peak in $\mathbf{5 5}$ was also used to support the C18-C19 relative assignment. We felt that the NOESY peaks shown for $\mathbf{5 5}$ were not unique for the $19 R$ (erythro C18-C19) stereochemical assignment of $\mathbf{5 5}$. It is possible that the C19-epimer of $\mathbf{5 5}$, compound $\mathbf{5 6}$ (19S instead of $19 R$ ), could exhibit the same NOESY peaks and coupling constant data. Therefore, we considered the possibility that C19 might have been originally misassigned.

Based upon the correlation experiments described above, we thought that we had confirmed the $\mathrm{C} 7, \mathrm{C} 8, \mathrm{C} 13, \mathrm{C} 16, \mathrm{C} 17$ and the $\mathbf{C 2}$ stereochemistry of both natural amphidinolide $\mathrm{E}$ as well as our synthetic intermediates. In addition, we felt that Kobayashi's C18 stereochemical assignment was irrefutable. Therefore, we concluded at this stage that the structure of natural amphidinolide E most likely was $\mathbf{5 7}$, with $19 S$ instead of $19 R$ stereochemistry (Figure 3).

\subsection{Synthesis of 2-epi-19-epi-amphidinolide E}

The $19 S$ stereochemistry in the possible alternative structure of amphidinolide E (57) was incorporated into our synthetic route by using $Z$ - $(S, S)$-crotylboronate $\mathbf{5 9}^{23}$ for the asymmetric crotylboration of L-glyceraldehyde pentylidene ketal 58. ${ }^{24}$ This experiment provided homoallylic alcohol $\mathbf{6 0}$ in good diastereoselectivity (Scheme 13). Elaboration of $\mathbf{6 0}$ into allylsilane $\mathbf{8}$ was accomplished using steps analogous to our original route shown previously in Scheme 2.

The [3+2] annulation reaction between allylsilane 8 ( 3 equiv) and aldehyde 5 provide 65 with $>20: 1$ selectivity in $61 \%$ yield (Scheme 14). The excess 8 can be recovered with excellent efficiency (92\%). The [3+2] adduct 65 was transformed into alcohol 67 using the same sequence as described for the synthesis of $\mathbf{2 5}$ (Scheme 4).

The C16-C17 relative stereochemistry of $\mathbf{8}$ was confirmed via basic Peterson elimination to afford the $Z$ diene $\mathbf{6 8}$ (Scheme 15). Assessment of the $\mathrm{C} 17$ absolute stereochemistry was attempted by direct esterification of the hydroxyl group in $\mathbf{8}$ with $(R)$ and $(S)$-MTPA-Cl. However, the hydroxyl group of $\mathbf{8}$ is quite hindered and no reaction was observed. Therefore, confirmation of the $\mathrm{C} 17$ absolute stereochemistry was accomplished by the Mosher ester analysis of alcohol 66.

Esterification of alcohol $\mathbf{6 7}$ with acid $\mathbf{1 0}$ afforded $\mathbf{7 0}$ as single diastereomer with complete inversion of the $\mathrm{C} 2$ stereochemistry (Scheme 16). Again, we had no reason to suspect inversion at $\mathrm{C} 2$ due to the high diastereoselectivity and the very clean "spot to spot" nature of the reaction. Elaboration of $\mathbf{7 0}$ into 2-epi-19-epi-amphidinolide E (4) was accomplished using the same chemistry as described for the synthesis of 2-epi-amphidinolide E (2) (Scheme 6).

However, it should be mentioned that a small amount of the presumed enyne side products was once again observed during the ring closing metathesis reaction (10\%). In addition, the acidic deprotection of $\mathbf{7 1}$ afforded a 2:1 inseparable mixture of the regioisomeric C18 (desired) and C17 lactones. Stille coupling of this mixture with vinylstannane $\mathbf{6}$ followed by HPLC separation of the isomers afforded pure 4. To our dismay, spectroscopic properties of 2-epi-19-epiamphidinolide E (4), which at this stage we thought was structure 57, once again did not match Kobayashi's data for natural amphidinolide E. 


\subsection{Discovery of the $C 2$ inversion problem and synthesis of amphidinolide $E$ (1) and 19-epi- amphidinolide $\mathrm{E}(3)$}

After synthesizing what we thought were structures 1 and $\mathbf{5 7}$ (i.e. syntheses of 2-epiamphidinolide E (2) and 2-epi-19-epi-amphidinolide E (4)) only to arrive at material that did not match Kobayashi's natural amphidinolide E, we decided to revisit the original $19 R$ series of compounds in order to obtain more insight into the true structure of amphidinolide $\mathrm{E}$ (reaction pathway B, Scheme 17). Critically, we had consumed all of our supply of acid diastereomer 10 and decided to use the large supply of diastereomer $\mathbf{9}$ that had accumulated in our laboratory (reaction pathway A, Scheme 17). Use of either acid diastereomer $\mathbf{9}$ or $\mathbf{1 0}$ should lead to polyene $\mathbf{7 2}$ after oxidative decomplexation of the $(\mathrm{CO})_{3} \mathrm{Fe}$ unit if $\mathrm{C} 2$ inversion were not occurring. To our surprise, we discovered that esterification-decomplexation reactions using $\mathbf{9}$ and $\mathbf{1 0}$ did not converge to one compound. Instead, they each separately yielded different polyenes $\mathbf{7 2}$ and $\mathbf{7 3}$ as single diastereomers. Before this discovery, we had always thought that the esterification-decomplexation sequence involving $\mathbf{1 0}$ was yielding polyene 72. Instead, it became absolutely clear based on the following examples that use of acid 10 in this sequence afforded polyene $\mathbf{7 3}$, proceeding through $\mathbf{3 9}$, with clean inversion at $\mathrm{C} 2$.

The discovery of separate, divergent reaction pathways for acids $\mathbf{9}$ and $\mathbf{1 0}$ prompted the evaluation of the $\mathrm{C} 2$ stereocenter in intermediates both prior to and after the esterification reaction (Table 2). In addition, the starting material used in the synthesis of both $\mathbf{9}$ and $\mathbf{1 0}$, oxazolidinone 36, was also evaluated. Table 2 summarizes these correlation experiments. Compounds 36, 9, 10, 76 and 39 were transformed into diene 75. The optical rotations of $\mathbf{7 5}$ from each reduction sequence were then compared with material independently synthesized from aldehyde $\mathbf{4 5},{ }^{49}$ using the method of Keck. ${ }^{53}$ The $2 R$ stereochemistry of $\mathbf{3 6}$ was verified prior to complexation of the $(\mathrm{CO})_{3} \mathrm{Fe}$ unit and hydrolysis of the acyloxazolidinone (entry 1 , Table 2). The $2 S$ stereochemistry of acids $\mathbf{9}$ and $\mathbf{1 0}$ was also verified prior to being subjected to the esterification reactions (entries $2 \& 3$, Table 2). Furthermore, the $\mathrm{C} 2$ stereochemistry of ester 76, the product of esterification of $\mathbf{2 5}$ with acid $\mathbf{9}$, was confirmed as $2 S$ (entry 4 , Table 2). On the other hand, the data in entry 5 indisputably affirms that inversion at $C 2$ occurs when acid $\mathbf{1 0}$ is used in the esterification reaction of $\mathbf{2 5}$.

We were pleased to find that subjection of polyene $\mathbf{7 2}$ to the same sequence of reactions used to synthesized 2-epi-amphidinolide E (2) and 2-epi-19-epi-amphidinolide E (4) afforded synthetic amphidinolide E (1), which had spectroscopic properties that matched natural amphidinolide $\mathrm{E}$ (Scheme 18). Interestingly, it should be noted that, unlike the 2-epi-series, the acidic deprotection of $\mathbf{7 7}$ only afforded the desired C18 lactone and none of the undesired $\mathrm{C} 17$ regioisomeric lactone. As observed before, a mixture of products thought to arrive by enyne metatesis was also isolated in $10 \%$ yield.

Furthermore, we also synthesized the final C2 and C19 stereochemical permutation, 19-epiamphinolide E (3) (Scheme 19). Only the C18 lactone was observed in the deprotection of 79. Furthermore, only a $5 \%$ yield of presumbed enyne products was observed during the RING CLOSING METATHESIS reaction. During the course of the synthesis of $\mathbf{3}$, we treated $\mathbf{7 8}$ with $\mathrm{K}_{2} \mathrm{CO}_{3}$ (0.9 equiv) in methanol at $50^{\circ} \mathrm{C}$ for $3 \mathrm{~h}$ and obtained a $2.6: 1$ mixture of $\mathrm{C} 2$ diastereomers favoring 78. This experiment establishes that the epimerizations observed in the esterifications of $\mathbf{2 5}$ (Scheme 6) and $\mathbf{6 7}$ (Scheme 16) with the diastereomeric $\mathrm{Fe}(\mathrm{CO})_{3^{-}}$ complexed dienoic acid $(2 S, 3 S)$-10 are contrathermodynamic, and therefore also kinetically controlled. 18

We hypothesize that ketene intermediates may be involved in the esterification reactions with (CO) ${ }_{3}$ Fe-complexed acids $\mathbf{9}$ and $\mathbf{1 0}$ (Scheme 20). Ketene intermediates have previously been implicated by Fürstner and co-workers in studies of the Yamaguchi macrolactonization 
directed towards iejimalide B. ${ }^{54}$ Activation of acid $\mathbf{1 0}$ followed by elimination could afford ketene intermediate 81. Addition of the alcohol coupling partner (HO-R) to $\mathbf{8 1}$ and subsequent reformation of the $\mathrm{C} 2$ stereocenter via diastereoselective protonation of enol $\mathbf{8 2}$ anti to the $(\mathrm{CO})_{3} \mathrm{Fe}$ unit would yield the observed $\mathrm{C} 2$ invert product $\mathbf{8 0}$. Alternatively, DMAP could add to the ketene intermediate $81 .{ }^{55}$ Diastereoselective protonation of enolate $\mathbf{8 3}$ followed by alkoxide addtion to acyl pyridinium $\mathbf{8 4}$ could also afford product $\mathbf{8 0}$.

Matching spectroscopic details for natural and synthetic amphidinolide $\mathrm{E}$ are summarized in Table 3 , confirming the validity of the originally assigned structure. Characteristic ${ }^{1} \mathrm{H}$ NMR data for all four amphidinolide stereoisomers (1-4) are presented in Table 4. It is noteworthy, that the $\mathrm{H} 3$ resonance is substantially shifted up field relative to amphidinolide $\mathrm{E}$ for both $\mathrm{C} 2$ epimers ( $\mathbf{2}$ and $\mathbf{4}$ ). Furthermore, the C30-Me resonance is also shifted up field for $\mathbf{2}$ and $\mathbf{4}$. On the other hand, the C29-Me is shifted downfield in the 19-epi-series ( $\mathbf{3}$ and $\mathbf{4})$. Only the data for synthetic 1 matches that of the natural product.

\section{Conclusion}

In conclusion, we have synthesized four stereoisomers of amphidinolide E, namely amphidinolide E (1), 2-epi-amphidinolide E (2), 19-epi-amphidinolide E (3), and 2-epi-19epi-amphidinolide $\mathrm{E}(\mathbf{4})$. This constitutes a rigorous verification of the stereochemistry of amphidinolide E. ${ }^{56}$ In the course of the studies toward $\mathbf{1}$, we discovered an unexpected and highly selective $\mathrm{C} 2$ inversion in the esterification reaction of $(\mathrm{CO})_{3} \mathrm{Fe}$-complexed dienoic acid 10. Insight into the possible mechanism of this epimerization, the context of which depends on the steric environment of the alcohol, has been published elsewhere. ${ }^{18}$ Results of the biological evaluation of $\mathbf{2}, \mathbf{3}$, and $\mathbf{4}$ will be reported in due course.

\section{Experimental}

\section{General Experimental Details}

All reaction solvents were purified before use. Tetrahydrofuran, dichloromethane, diethyl ether, and toluene were purified by passing through a solvent column composed of activated A-1 alumina. Unless indicated otherwise, all reactions were conducted under an atmosphere of argon using flame-dried or oven-dried $\left(170^{\circ} \mathrm{C}\right)$ glassware. Four $\AA$ molecular sieves were activated under high vacuum with heat $\left(180^{\circ} \mathrm{C}\right)$ for $12 \mathrm{~h}$ and re-activated by thorough flamedrying immediately prior to use.

Proton nuclear magnetic resonance $\left({ }^{1} \mathrm{H}\right.$ NMR) spectra were recorded on commercial instruments at 400 or $500 \mathrm{MHz}$. Carbon-13 nuclear magnetic resonance $\left({ }^{13} \mathrm{C} \mathrm{NMR}\right)$ spectra were recorded at 100 and $125 \mathrm{MHz}$, respectively. The proton signal for residual non-deuterated solvent $\left(\delta 7.26\right.$ for $\mathrm{CHCl}_{3}$ ) was used as an internal reference for ${ }^{1} \mathrm{H}$ NMR spectra. For ${ }^{13} \mathrm{C}$ NMR spectra, chemical shifts are reported relative to the $\delta 77.2$ resonance of $\mathrm{CHCl}_{3}$. Coupling constants are reported in Hz. Infrared (IR) spectra were recorded as films on a FTIR instrument. Opitcal rotations were measured on a polarimeter using a quartz cell with $1 \mathrm{~mL}$ capacity and a $10 \mathrm{~cm}$ path length. Mass spectra were recorded on a commercial spectrometer.

Analytical thin layer chromatography (TLC) was performed on Kieselgel $60 \mathrm{~F}_{254}$ glass plates pre-coated with a $0.25 \mathrm{~mm}$ thickness of silica gel. The TLC plates were visualized with UV light and/or by staining with Hanessian solution (ceric sulfate and ammonium molybdate in aqueous sulfuric acid). Column chromatography was generally performed using Kieselgel 60 (230-400 mesh) silica gel, typically using a 50-100:1 weight ratio of silica gel to crude product.

HPLC purifications were performed by using a HPLC system composed of two Varian Prostar pumps (model 210) connected to normal phase columns. Samples were loaded into the system 
with a $2 \mathrm{~mL}$ Rheodyne 7125 injector and were detected using a Varian Prostar UV and a Varian RI detector.

\section{(E)-5-((4R,5R)-2,2-Dimethyl-5-vinyl-[1,3]dioxolan-4-yl)-pent-4-enoic acid methyl ester (15)}

To a $-78^{\circ} \mathrm{C}$ solution of $(\mathrm{COCl})_{2}(3.45 \mathrm{~mL}, 39.4 \mathrm{mmol})$ in $\mathrm{CH}_{2} \mathrm{Cl}_{2}(80 \mathrm{~mL})$ was added DMSO ( $3.50 \mathrm{~mL}, 49.2 \mathrm{mmol})$ in $\mathrm{CH}_{2} \mathrm{Cl}_{2}(10 \mathrm{~mL})$. The reaction was stirred at $-78{ }^{\circ} \mathrm{C}$ for $15 \mathrm{~min}$, then alcohol $14^{20}(3.12 \mathrm{~g}, 19.7 \mathrm{mmol})$ in $\mathrm{CH}_{2} \mathrm{Cl}_{2}(10 \mathrm{~mL})$ was added. The reaction was stirred for $20 \mathrm{~min}$ at $-78^{\circ} \mathrm{C}$ followed by the addition of triethylamine $(16.4 \mathrm{~mL}, 118 \mathrm{mmol})$. The mixture was allowed to warm to $0^{\circ} \mathrm{C}$. After $30 \mathrm{~min}$, the reaction was diluted with $\mathrm{Et}_{2} \mathrm{O}(300 \mathrm{~mL})$, upon which a white precipitate forms (triethylamine hydrochloride). The slurry was filtered through a 1 inch pad of Celite and concentrated to afford the aldehyde, a yellow oil, which was immediately used in the next reaction.

To a $0{ }^{\circ} \mathrm{C}$ solution of the crude aldehyde in THF $(60 \mathrm{~mL})$ was added vinylmagnesium bromide ( $60 \mathrm{~mL}$ of a $1.0 \mathrm{M}$ THF solution, $60 \mathrm{mmol}$ ). The reaction was stirred for $2.5 \mathrm{~h}$, and then quenched with saturated aqueous $\mathrm{NaHCO}_{3}(50 \mathrm{~mL})$ and extract with $\mathrm{Et}_{2} \mathrm{O}(20 \mathrm{~mL} \times 3)$. The organic phase was washed with brine $(50 \mathrm{~mL})$, dried over anhydrous $\mathrm{MgSO}_{4}$, filtered, and concentrated to afford a mixture of diastereomeric allylic alcohols as a yellow oil. This oil was used immediately in the next reaction.

To the mixture of diastereomeric allylic alcohols, from the preceding step, in toluene (66 mL) was added trimethyl orthoacetate $(12.5 \mathrm{~mL}, 98.5 \mathrm{mmol})$ and propionoic acid $(0.3 \mathrm{~mL}, 3.94$ $\mathrm{mmol}$ ). The reaction was fitted with a condenser and placed in a $110^{\circ} \mathrm{C}$ oil bath for $18 \mathrm{~h}$. The solution was then quenched with $3 \mathrm{~mL}$ of triethylamine and concentrated. The crude product was purified by flash column chromatography to yield methyl ester $\mathbf{1 5}(2.83 \mathrm{~g}, 60 \%$ over 3 steps) as a colorless oil: $[\alpha]^{25} \mathrm{D}=-132^{\circ}\left(c 0.99, \mathrm{CHCl}_{3}\right) ;{ }^{1} \mathrm{H} \mathrm{NMR}\left(400 \mathrm{MHz}, \mathrm{CDCl}_{3}\right) \delta$ 5.74-5.83 (m, 2H), $5.48(\mathrm{dd}, J=6.4,15.2 \mathrm{~Hz}, 1 \mathrm{H}), 5.33(\mathrm{~d}, J=17.2 \mathrm{~Hz}, 1 \mathrm{H}), 5.24(\mathrm{~d}, J=10.4$ $\mathrm{Hz}, 1 \mathrm{H}), 4.04$ (app q, $J=6.8 \mathrm{~Hz}, 2 \mathrm{H}), 3.67(\mathrm{~s}, 3 \mathrm{H}), 2.36-2.44(\mathrm{~m}, 4 \mathrm{H}), 1.44(\mathrm{~s}, 3 \mathrm{H}), 1.43$ (s, $3 \mathrm{H}) ;{ }^{13} \mathrm{C}$ NMR $\left(100 \mathrm{MHz}, \mathrm{CDCl}_{3}\right) \delta 172.8,134.0,133.7,126.9,118.3,108.7,82.0,81.6,51.3$, 33.1, 27.3, 26.8, 26.7; IR (neat) 2987, 2874, 1740, 1437, $1371 \mathrm{~cm}^{-1}$; HRMS (ES+) $\mathrm{m} / z$ for $\mathrm{C}_{12} \mathrm{H}_{18} \mathrm{O}_{3} \mathrm{Na}[\mathrm{M}+\mathrm{Na}]^{+}$calcd 263.1259, found 263.1255.

\section{(E)-5-((4R,5R)-2,2-Dimethyl-5-vinyl-[1,3]dioxolan-4-yl)-pent-4-enal (5)}

To a $-78{ }^{\circ} \mathrm{C}$ solution of methyl ester $15(2.25 \mathrm{~g}, 9.36 \mathrm{mmol})$ in toluene $(31 \mathrm{~mL})$ was added DIBAL $(9.36 \mathrm{~mL}$ of a $1.0 \mathrm{M}$ hexane solution, $9.36 \mathrm{mmol})$ dropwise such that the internal temperature was below $-70^{\circ} \mathrm{C}$. After being stirred for $30 \mathrm{~min}$, the reaction was quenched with saturated aqueous sodium potassium tartrate (Rochelle's salt) $(40 \mathrm{~mL})$ and diluted with $\mathrm{Et}_{2} \mathrm{O}$ $(20 \mathrm{~mL})$. The mixure was stirred at room temperature for $3 \mathrm{~h}$ and extracted with $\mathrm{Et}_{2} \mathrm{O}(20 \mathrm{~mL}$ $\times 3$ ). The organic phase was washed with brine $(50 \mathrm{~mL})$, dried over anhydrous $\mathrm{MgSO}_{4}$, filtered, and concentrated. The crude product was purified by flash column chromatography to afford aldehyde $5(1.59 \mathrm{~g}, 81 \%)$ as a colorless oil: $[\alpha]^{25} \mathrm{D}=-28.7^{\circ}\left(c 1.41, \mathrm{CHCl}_{3}\right) ;{ }^{1} \mathrm{H}$ NMR $\left(400 \mathrm{MHz}, \mathrm{CDCl}_{3}\right) \delta 9.73(\mathrm{bs}, 1 \mathrm{H}), 5.70-5.85(\mathrm{~m}, 2 \mathrm{H}), 5.49$ (bdd, $\left.J=6.0,15.6 \mathrm{~Hz}, 1 \mathrm{H}\right), 5.34$ $(\mathrm{d}, J=17.2 \mathrm{~Hz}, 1 \mathrm{H}), 5.24(\mathrm{~d}, J=10.4 \mathrm{~Hz}, 1 \mathrm{H}), 4.00-4.10(\mathrm{~m}, 2 \mathrm{H}), 2.52-2.60(\mathrm{~m}, 2 \mathrm{H}), 2.35-2.45$ $(\mathrm{m}, 2 \mathrm{H}), 1.44(\mathrm{~s}, 3 \mathrm{H}), 1.43(\mathrm{~s}, 3 \mathrm{H}) ;{ }^{13} \mathrm{C}$ NMR $\left(100 \mathrm{MHz}, \mathrm{CDCl}_{3}\right) \delta 201.3,134.1,133.8,127.2$, 118.8, 109.0, 82.2, 81.8, 42.8, 27.0, 27.0, 24.7; IR (neat) 3085, 2987, 2875, 1726, 1379, 1371, $1239 \mathrm{~cm}^{-1}$; HRMS (ES+) $\mathrm{m} / z$ for $\mathrm{C}_{13} \mathrm{H}_{20} \mathrm{O}_{3} \mathrm{Na}[\mathrm{M}+\mathrm{Na}]^{+}$calcd 233.1154, found 233.1245.

\section{(3R,4R)-4-((S)-2,2-Diethyl-[1,3]dioxolan-4-yl)-4-(4-methoxy-benzyloxy)-3-methyl-butan-1-ol} (17)

To a $0{ }^{\circ} \mathrm{C}$ slurry of $\mathrm{NaH}(1.69 \mathrm{~g}, 70.6 \mathrm{mmol})$ and $\mathrm{Bu}_{4} \mathrm{NI}(1.7 \mathrm{~g}, 4.7 \mathrm{mmol})$ in THF $(157 \mathrm{~mL})$ was added homoallylic alcohol $\mathbf{1 6}^{22}(10.1 \mathrm{~g}, 47.0 \mathrm{mmol})$ followed by $p$-methoxybenzyl chloride $(6.38 \mathrm{~mL}, 47.0 \mathrm{mmol})$. The reaction was fitted with a condenser and refluxed for 16 
h. The reaction was quenched with sat. aq. $\mathrm{NH}_{4} \mathrm{Cl}(50 \mathrm{~mL})$ and water $(50 \mathrm{~mL})$ and extracted with EtOAc $(25 \mathrm{~mL} \times 3)$. The organic phase was washed with brine, dried over anhydrous $\mathrm{MgSO}_{4}$, filtered and concentrated. The crude product was purified by flash column chromatography to afforded the $p$-methoxybenzyl ether $(15.15 \mathrm{~g}, 96 \%)$ as a colorless oil: $[\alpha]$ $25_{\mathrm{D}}=-41^{\circ}\left(c 1.53, \mathrm{CHCl}_{3}\right) ;{ }^{1} \mathrm{H} \mathrm{NMR}\left(400 \mathrm{MHz}, \mathrm{CDCl}_{3}\right) \delta 7.25(\mathrm{~d}, J=8.4 \mathrm{~Hz}, 2 \mathrm{H}), 6.87(\mathrm{~d}$, $J=8.4 \mathrm{~Hz}, 2 \mathrm{H}), 5.86(\mathrm{ddd}, J=8.0,10.4,17.2 \mathrm{~Hz}, 1 \mathrm{H}), 5.04($ app t, $J=17.6 \mathrm{~Hz}, 2 \mathrm{H}), 4.60(\mathrm{AB}$, $J=10.8 \mathrm{~Hz}, 1 \mathrm{H}), 4.55(\mathrm{AB}, J=11.2 \mathrm{~Hz}, 2 \mathrm{H}), 4.05-4.10(\mathrm{~m}, 1 \mathrm{H}), 4.00(\mathrm{dd}, J=6.0,7.6 \mathrm{~Hz}$, $1 \mathrm{H}), 3.81(\mathrm{~s}, 3 \mathrm{H}), 3.77(\mathrm{~d}, J=7.6 \mathrm{~Hz}, 1 \mathrm{H}), 3.52(\mathrm{dd}, J=3.6,6.0 \mathrm{~Hz}, 1 \mathrm{H}), 2.50-2.54(\mathrm{~m}, 1 \mathrm{H})$, $1.57-1.70(\mathrm{~m}, 4 \mathrm{H}), 1.09(\mathrm{~d}, J=6.8 \mathrm{~Hz}, 3 \mathrm{H}), 0.89(\mathrm{dt}, J=9.6,7.6 \mathrm{~Hz}, 6 \mathrm{H}) ;{ }^{13} \mathrm{C} \mathrm{NMR}(100 \mathrm{MHz}$, $\left.\mathrm{CDCl}_{3}\right) \delta 159.1,140.1,130.1,129.3,115.0,113.7,112.1,83.1,77.3,74.0,66.9,55.2,40.8$, 29.7, 29.0, 17.0, 8.2, 8.1; IR (neat) 3073, 2972, 1613, 1514, $1249 \mathrm{~cm}^{-1}$; HRMS (ES+) $\mathrm{m} / z$ for $\mathrm{C}_{20} \mathrm{H}_{30} \mathrm{O}_{4} \mathrm{Na}[\mathrm{M}+\mathrm{Na}]^{+}$calcd 357.2042, found 357.2044.

To a solution of the $p$-methoxybenzyl ether $(15.1 \mathrm{~g}, 45.3 \mathrm{mmol})$ in THF $(181 \mathrm{~mL})$ was added 9-BBN ( $272 \mathrm{~mL}$ of a $0.5 \mathrm{M}$ THF solution, $136 \mathrm{mmol})$. The reaction was fitted with a condenser, refluxed for $3 \mathrm{~h}$, cooled to $0{ }^{\circ} \mathrm{C}$ and quenched with water $(25 \mathrm{~mL})$. The mixure was then treated with $2 \mathrm{~N} \mathrm{NaOH}$ aq. $\left(227 \mathrm{~mL}\right.$ ) followed by $30 \%(\mathrm{w} / \mathrm{w}) \mathrm{H}_{2} \mathrm{O}_{2}(46.3 \mathrm{~mL})$ and the biphasic mixture was stirred at room temperature for $17 \mathrm{~h}$. The aqueous phase was extracted with EtOAc (50 $\mathrm{mL} \times 3$ ). The organic phase was washed with brine, dried over anhydrous $\mathrm{MgSO}_{4}$, filtered and concentrated. The crude product was purified by flash column chromatography to afford $\mathbf{1 7}$ $(15.1 \mathrm{~g}, 94 \%)$ as a colorless oil: $[\alpha]^{25} \mathrm{D}=-27^{\circ}\left(c 0.63, \mathrm{CHCl}_{3}\right) ;{ }^{1} \mathrm{H} \mathrm{NMR}\left(500 \mathrm{MHz}, \mathrm{CDCl}_{3}\right)$ $\delta 7.24(\mathrm{~d}, J=8.5 \mathrm{~Hz}, 2 \mathrm{H}), 6.87(\mathrm{~d}, J=8.4 \mathrm{~Hz}, 2 \mathrm{H}), 4.56(\mathrm{~s}, 2 \mathrm{H}), 4.16(\operatorname{app~q}, J=6.5 \mathrm{~Hz}, 1 \mathrm{H})$, $4.07(\mathrm{dd}, J=6.0,8.0 \mathrm{~Hz}, 1 \mathrm{H}), 3.80(\mathrm{~s}, 3 \mathrm{H}), 3.75(\mathrm{app} \mathrm{t}, J=8.0 \mathrm{~Hz}, 1 \mathrm{H}), 3.70-3.76(\mathrm{~m}, 1 \mathrm{H})$, $3.60-3.64(\mathrm{~m}, 1 \mathrm{H}), 3.46(\mathrm{dd}, J=4.5,6.0 \mathrm{~Hz}, 1 \mathrm{H}), 2.02-2.07(\mathrm{~m}, 1 \mathrm{H}), 1.95(\mathrm{dd}, J=4.5,6.0 \mathrm{~Hz}$, $1 \mathrm{H}), 1.73-1.79(\mathrm{~m}, 1 \mathrm{H}), 1.58-1.67(\mathrm{~m}, 4 \mathrm{H}), 1.03(\mathrm{~d}, J=7.0 \mathrm{~Hz}, 3 \mathrm{H}), 0.89(\mathrm{dt}, J=7.0,5.5 \mathrm{~Hz}$, $6 \mathrm{H}) ;{ }^{13} \mathrm{C}$ NMR $\left(125 \mathrm{MHz}, \mathrm{CDCl}_{3}\right) \delta 159.2,130.3,129.3,113.7,112.6,83.3,77.3,76.3,67.7$, 60.5, 55.2, 34.9, 32.1, 29.7, 29.0, 16.3, 8.2; IR (neat) 3436, 2971, 2881, 1613, 1514, 1249 $\mathrm{cm}^{-1}$; HRMS (ES+) $\mathrm{m} / z$ for $\mathrm{C}_{20} \mathrm{H}_{32} \mathrm{O}_{5} \mathrm{Na}[\mathrm{M}+\mathrm{Na}]^{+}$calcd 375.2147, found 375.2141.

\section{(S)-2,2-Diethyl-4-[(1R,2R)-1-(4-methoxy-benzyloxy)-2-methyl-pent-4-ynyl]-[1,3]dioxolane} (18)

To a $0{ }^{\circ} \mathrm{C}$ solution of alcohol $17(15.0 \mathrm{~g}, 42.6 \mathrm{mmol})$ in $\mathrm{CH}_{2} \mathrm{Cl}_{2}(142 \mathrm{~mL})$ was added DMSO (9.1 mL, $128 \mathrm{mmol}), i-\mathrm{Pr}_{2} \mathrm{NEt}(22.2 \mathrm{~mL}, 128 \mathrm{mmol})$ and $\mathrm{SO}_{3} \cdot \operatorname{Pyr}(20.3 \mathrm{~g}, 128 \mathrm{mmol})$. The reaction was stirred at $0{ }^{\circ} \mathrm{C}$ for $30 \mathrm{~min}$, then quenched with sat. aq. $\mathrm{Na}_{2} \mathrm{~S}_{2} \mathrm{O}_{3}(100 \mathrm{~mL})$ and extracted with $\mathrm{CH}_{2} \mathrm{Cl}_{2}(30 \mathrm{~mL} \times 3)$. The organic phase was washed with brine, dried over anhydrous $\mathrm{MgSO}_{4}$, filtered and concentrated. The crude product was purified by flash column chromatography to afford the aldehyde $(13.39 \mathrm{~g}, 89 \%)$ as a colorless oil: $[\alpha]^{25} \mathrm{D}=-30^{\circ}(c 2.2$, $\left.\mathrm{CHCl}_{3}\right) ;{ }^{1} \mathrm{H} \mathrm{NMR}\left(500 \mathrm{MHz}, \mathrm{CDCl}_{3}\right) \delta 9.73(\mathrm{app} \mathrm{t}, J=2.0 \mathrm{~Hz}, 1 \mathrm{H}), 7.23(\mathrm{~d}, J=9.0 \mathrm{~Hz}, 2 \mathrm{H})$, $6.87(\mathrm{~d}, J=9.0 \mathrm{~Hz}, 2 \mathrm{H}), 4.56(\mathrm{AB}, J=11.0 \mathrm{~Hz}, 1 \mathrm{H}), 4.53(\mathrm{AB}, J=11.0 \mathrm{~Hz}, 1 \mathrm{H}), 4.12(\mathrm{dd}$, $J=6.5,13.0 \mathrm{~Hz}, 1 \mathrm{H}), 4.06(\mathrm{dd}, J=6.5,8.0 \mathrm{~Hz}, 1 \mathrm{H}), 3.80(\mathrm{~s}, 3 \mathrm{H}), 3.73(\mathrm{t}, J=8.0 \mathrm{~Hz}, 1 \mathrm{H}), 3.40$ (dd, $J=4.5,6.0 \mathrm{~Hz}, 1 \mathrm{H}), 2.65$ (ddd, $J=2.0,6.0,7.5 \mathrm{~Hz}, 1 \mathrm{H}), 2.43-2.48(\mathrm{~m}, 1 \mathrm{H}), 2.37$ (ddd, $J=2.0,7.5,9.5 \mathrm{~Hz}, 1 \mathrm{H}), 1.57-1.67(\mathrm{~m}, 4 \mathrm{H}), 1.06(\mathrm{~d}, J=7.5, \mathrm{~Hz}, 3 \mathrm{H}), 0.88$ (t, $J=7.0 \mathrm{~Hz}$, $6 \mathrm{H}) ;{ }^{13} \mathrm{C}$ NMR $\left(125 \mathrm{MHz}, \mathrm{CDCl}_{3}\right) \delta 202.2,159.2,130.2,129.4,113.7,113.0,82.2,76.1,73.3$, 67.6, 55.2, 47.1, 30.3, 29.7, 28.9, 16.5, 8.2, 8.2; IR (neat) 2971, 2934, 2724, 2721, 1724, 1514, $1249 \mathrm{~cm}^{-1}$; HRMS (ES+) $\mathrm{m} / z$ for $\mathrm{C}_{20} \mathrm{H}_{30} \mathrm{O}_{5} \mathrm{Na}[\mathrm{M}+\mathrm{Na}]^{+}$calcd 373.1991, found 373.1984 .

To a $0{ }^{\circ} \mathrm{C}$ solution of $\mathrm{PPh}_{3}(24.9 \mathrm{~g}, 94.87 \mathrm{mmol})$ in $\mathrm{CH}_{2} \mathrm{Cl}_{2}(182 \mathrm{~mL})$ was added $\mathrm{CBr}_{4}(15.7$ $\mathrm{g}, 47.4 \mathrm{mmol})$. The reaction was warmed to room temperature for $30 \mathrm{~min}$ and then cooled back to $0{ }^{\circ} \mathrm{C}$. To this mixture was added the aldehyde from the preceding step $(12.8 \mathrm{~g}, 36.5 \mathrm{mmol})$ in $\mathrm{CH}_{2} \mathrm{Cl}_{2}(5 \mathrm{~mL})$. The reaction was stirred for $30 \mathrm{~min}$ and then diluted with hexane $(400 \mathrm{~mL})$, upon which a white precipitate formed $\left(\mathrm{Ph}_{3} \mathrm{P}=\mathrm{O}\right)$. The slurry was filtered through Celite and concentrated. The residue was dissolve in hexane $(300 \mathrm{~mL})$ to precipitate more $\mathrm{Ph}_{3} \mathrm{P}=\mathrm{O}$. The 
slurry was filtered through Celite and again concentrated. The residual oil was dissolved in THF $(100 \mathrm{~mL})$, cooled to $-78^{\circ} \mathrm{C}$ and treated with $\mathrm{n}-\mathrm{BuLi}(32.4 \mathrm{~mL}$ of $2.29 \mathrm{M}$ hexane solution, $74.3 \mathrm{mmol})$. The reaction was stirred for $1 \mathrm{~h}$ and then quenched with sat. aq. $\mathrm{NH}_{4} \mathrm{Cl}(100 \mathrm{~mL})$ and extracted with EtOAc $(50 \mathrm{~mL} \times 3)$. The organic phase was washed with brine, dried over anhydrous $\mathrm{MgSO}_{4}$, filtered and concentrated. Purification of the crude product by flash column chromatography afforded $\mathbf{1 8}(11.0 \mathrm{~g}, 98 \%)$ as a colorless oil: $[\alpha]^{25} \mathrm{D}=-7.6^{\circ}(c 0.89$,

$\left.\mathrm{CHCl}_{3}\right) ;{ }^{1} \mathrm{H} \mathrm{NMR}\left(400 \mathrm{MHz}, \mathrm{CDCl}_{3}\right) \delta 7.25(\mathrm{~d}, J=8.8 \mathrm{~Hz}, 2 \mathrm{H}), 6.87(\mathrm{~d}, J=8.8 \mathrm{~Hz}, 2 \mathrm{H}), 4.62$ $(\mathrm{AB}, J=10.8 \mathrm{~Hz}, 1 \mathrm{H}), 4.54(\mathrm{AB}, J=11.2 \mathrm{~Hz}, 1 \mathrm{H}), 4.17(\mathrm{dt}, J=6.0,8.0 \mathrm{~Hz}, 1 \mathrm{H}), 4.03(\mathrm{dd}$, $J=6.0,8.0 \mathrm{~Hz}, 1 \mathrm{H}), 3.80(\mathrm{~s}, 3 \mathrm{H}), 3.77(\mathrm{t}, J=8.0 \mathrm{~Hz}, 1 \mathrm{H}), 3.57(\mathrm{t}, J=6.0 \mathrm{~Hz}, 1 \mathrm{H}), 2.27-2.39$ $(\mathrm{m}, 2 \mathrm{H}), 1.98(\operatorname{app~t}, J=3.2 \mathrm{~Hz}, 1 \mathrm{H}), 1.91-1.98(\mathrm{~m}, 1 \mathrm{H}), 1.56-1.71(\mathrm{~m}, 4 \mathrm{H}), 1.10(\mathrm{~d}, J=7.2$ $\mathrm{Hz}, 3 \mathrm{H}), 0.90$ (app q, $J=7.6 \mathrm{~Hz}, 6 \mathrm{H}) ;{ }^{13} \mathrm{C}$ NMR $\left(100 \mathrm{MHz}, \mathrm{CDCl}_{3}\right) \delta 159.2,130.5,129.4$, 113.7, 112.8, 83.2, 81.2, 76.5, 73.7, 69.4, 67.0, 55.2, 34.9, 29.7, 29.0, 22.1, 15.7, 8.2, 8.1; IR (neat) $3295,2971,1613,1514 \mathrm{~cm}^{-1}$; HRMS (ES+) $\mathrm{m} / z$ for $\mathrm{C}_{21} \mathrm{H}_{30} \mathrm{O}_{4} \mathrm{Na}[\mathrm{M}+\mathrm{Na}]^{+}$calcd 369.2042, found 369.2037 .

\section{(2R,3R)-2-(4-Methoxy-benzyloxy)-3-methyl-hex-5-ynal (19)}

To alkyne 18 (4.84 g, $14.0 \mathrm{mmol})$ was added a 4:1 mixture of $\mathrm{AcOH}$ and water $(47 \mathrm{~mL})$. The reaction mixture was heated to $40{ }^{\circ} \mathrm{C}$ for $6 \mathrm{~h}$ and then was diluted with $50 \mathrm{~mL}$ of EtOAc. Solid $\mathrm{NaHCO}_{3}(20 \mathrm{~g})$ was slowly added portionwise and then the mixture was extracted with EtOAc $(25 \mathrm{~mL} \times 3)$. The organic phase was washed with brine, dried over anhydrous $\mathrm{MgSO}_{4}$, filtered and concentrated. The crude product was purified by flash column chromatography to afford the diol $(3.39 \mathrm{~g}, 87 \%)$ as a colorless oil: $[\alpha]^{25} \mathrm{D}=+13.6^{\circ}\left(c 0.59, \mathrm{CHCl}_{3}\right) ;{ }^{1} \mathrm{H} \mathrm{NMR}(400 \mathrm{MHz}$, $\left.\mathrm{CDCl}_{3}\right) \delta 7.27(\mathrm{~d}, J=8.8 \mathrm{~Hz}, 2 \mathrm{H}), 6.89(\mathrm{~d}, J=8.8 \mathrm{~Hz}, 2 \mathrm{H}), 4.64(\mathrm{AB}, J=11.2 \mathrm{~Hz}, 1 \mathrm{H}), 4.61$ $(\mathrm{AB}, J=11.2 \mathrm{~Hz}, 1 \mathrm{H}), 3.80(\mathrm{~s}, 3 \mathrm{H}), 3.69-3.84(\mathrm{~m}, 3 \mathrm{H}), 3.58(\mathrm{dd}, J=4.4,7.2 \mathrm{~Hz}, 1 \mathrm{H}), 2.33-2.46$ $(\mathrm{m}, 3 \mathrm{H}), 2.18(\mathrm{dd}, J=4.0,8.0 \mathrm{~Hz}, 1 \mathrm{H}), 2.03(\mathrm{t}, J=2.4 \mathrm{~Hz}, 1 \mathrm{H}), 1.96-2.02(\mathrm{~m}, 1 \mathrm{H}), 7.27(\mathrm{~d}$, $J=8.8 \mathrm{~Hz}, 2 \mathrm{H}), 1.08(\mathrm{~d}, J=6.8 \mathrm{~Hz}, 3 \mathrm{H}) ;{ }^{13} \mathrm{C} \mathrm{NMR}\left(100 \mathrm{MHz}, \mathrm{CDCl}_{3}\right) \delta 159.4,130.3,129.6$, 113.9, 83.8, 83.0, 74.8, 71.5, 69.9, 63.3, 55.3, 34.4, 21.9, 16.3; IR (neat) 3413, 3306, 2936, $1612,1515,1249 \mathrm{~cm}^{-1}$; HRMS (ES+) $\mathrm{m} / z$ for $\mathrm{C}_{16} \mathrm{H}_{22} \mathrm{O}_{4} \mathrm{Na}[\mathrm{M}+\mathrm{Na}]^{+}$calcd 301.1416 , found 301.1416.

To a $0{ }^{\circ} \mathrm{C}$ solution of the diol $(3.39 \mathrm{~g}, 12.2 \mathrm{mmol})$ in THF $(20 \mathrm{~mL})$ and $\mathrm{pH} 7$ buffer $(20 \mathrm{~mL})$ was added $\mathrm{NaIO}_{4}(3.13 \mathrm{~g}, 14.6 \mathrm{mmol})$. The reaction was stirred for $4 \mathrm{~h}$, quenched with sat. aq. $\mathrm{Na}_{2} \mathrm{~S}_{2} \mathrm{O}_{3}(25 \mathrm{~mL})$ and extracted with EtOAc $(25 \mathrm{~mL} \times 3)$. The organic phase was washed with brine, dried over anhydrous $\mathrm{MgSO}_{4}$, filtered and concentrated to afford pure $19(2.76 \mathrm{~g}, 92 \%)$ as a colorless oil: $[\alpha]^{25} \mathrm{D}=+80^{\circ}\left(c 2.26, \mathrm{CHCl}_{3}\right) ;{ }^{1} \mathrm{H} \mathrm{NMR}\left(500 \mathrm{MHz}, \mathrm{CDCl}_{3}\right) \delta 9.65$ (app d, $J=3.0 \mathrm{~Hz}, 1 \mathrm{H}), 7.27(\mathrm{~d}, J=8.5 \mathrm{~Hz}, 2 \mathrm{H}), 6.89(\mathrm{~d}, J=8.5 \mathrm{~Hz}, 2 \mathrm{H}), 4.59(\mathrm{~d}, J=11.5 \mathrm{~Hz}, 1 \mathrm{H})$, $4.47(\mathrm{~d}, J=11.5 \mathrm{~Hz}, 1 \mathrm{H}), 3.81(\mathrm{~s}, 3 \mathrm{H}), 3.60(\mathrm{dd}, J=3.0,10.0 \mathrm{~Hz}, 1 \mathrm{H}), 2.34-2.36(\mathrm{~m}, 2 \mathrm{H})$, 2.11-2.17 (m, 1H), $1.98($ app t, $J=2.5 \mathrm{~Hz}, 1 \mathrm{H}), 1.04(\mathrm{~d}, J=7.0 \mathrm{~Hz}, 3 \mathrm{H}) ;{ }^{13} \mathrm{C}$ NMR $(125 \mathrm{MHz}$, $\left.\mathrm{CDCl}_{3}\right) \delta 203.5,159.5,129.8,129.2,113.8,85.6,81.9,72.8,70.4,55.2,34.0,21.3,15.3$; IR (neat) $3292,2967,2837,1731,1515,1249 \mathrm{~cm}^{-1}$; HRMS (ES+) $\mathrm{m} / 2$ for $\mathrm{C}_{15} \mathrm{H}_{18} \mathrm{O}_{3} \mathrm{Na}[\mathrm{M}$ $+\mathrm{Na}]^{+}$calcd 269.1154, found 269.1147 .

\section{(3R,4S,5R,6R)-3-(Dimethylphenylsilanyl)-5-(4-methoxy-benzyloxy)-6-methyl-non-1-en-8- yn-4-ol (21)}

To a $-78{ }^{\circ} \mathrm{C}$ slurry of aldehyde $\mathbf{1 9}(5.95 \mathrm{~g}, 24.2 \mathrm{mmol})$ and $4 \AA$ mol. sieves $(4.8 \mathrm{~g})$ in toluene $\left(20 \mathrm{~mL}\right.$ ) was added $(S, S)-\mathbf{2 0}^{27}$ (61 mL of a 1.0M solution in toluene, $\left.60.4 \mathrm{mmol}\right)$. The reaction was stirred at $-78^{\circ} \mathrm{C}$ for $18 \mathrm{~h}$ and then quenched with $2 \mathrm{~N} \mathrm{NaOH}$ aq. $(100 \mathrm{~mL})$. The biphasic mixture was filtered through Celite and extracted with EtOAc $(30 \mathrm{~mL} \times 3)$. The organic phase was washed with brine, dried over anhydrous $\mathrm{MgSO}_{4}$, filtered and concentrated. The crude product was purified by flash column chromatography to afford $\mathbf{2 1}(9.19 \mathrm{~g}, 90 \%)$ as a colorless oil: $[\alpha]^{25}{ }_{\mathrm{D}}=-6^{\circ}\left(\mathrm{c} 2.48, \mathrm{CHCl}_{3}\right) ;{ }^{1} \mathrm{H}$ NMR $\left(400 \mathrm{MHz}, \mathrm{CDCl}_{3}\right) \delta$ 7.55-7.57 $(\mathrm{m}, 2 \mathrm{H}), 7.34-7.36$ $(\mathrm{m}, 3 \mathrm{H}), 7.25(\mathrm{~d}, J=8.8 \mathrm{~Hz}, 2 \mathrm{H}), 6.88(\mathrm{~d}, J=8.8 \mathrm{~Hz}, 2 \mathrm{H}), 5.98(\mathrm{dt}, J=10.4,21.5 \mathrm{~Hz}, 1 \mathrm{H})$, 
$5.03(\mathrm{~d}, J=10.4 \mathrm{~Hz}, 1 \mathrm{H}), 4.85(\mathrm{~d}, J=21.5 \mathrm{~Hz}, 1 \mathrm{H}), 4.58(\mathrm{~d}, J=13.0 \mathrm{~Hz}, 1 \mathrm{H}), 4.49(\mathrm{~d}, J=$ $13.5 \mathrm{~Hz}, 1 \mathrm{H}), 3.81(\mathrm{~s}, 3 \mathrm{H}), 3.73-3.77(\mathrm{~m}, 1 \mathrm{H}), 3.31(\mathrm{dd}, J=3.2,6.8 \mathrm{~Hz}, 1 \mathrm{H}), 2.43(\mathrm{~d}, J=4.0$ $\mathrm{Hz}, 1 \mathrm{H}), 2.08-2.18(\mathrm{~m}, 1 \mathrm{H}), 1.91-1.98(\mathrm{~m}, 3 \mathrm{H}), 1.08$ (d, J = 7.2 Hz, 3H), 0.39 (s, 3H), 0.34 (s, $3 \mathrm{H}) ;{ }^{13} \mathrm{C} \mathrm{NMR}\left(100 \mathrm{MHz}, \mathrm{CDCl}_{3}\right) \delta 159.3,137.9,134.8,134.1,130.4,129.4,129.0,127.6$, $114.5,113.9,85.5,83.6,74.9,71.1,69.3,55.3,39.2,34.1,20.3,17.9,-3.8,-4.2$; IR (neat) 3560, 3304, 2961, 1613, $1514 \mathrm{~cm}^{-1}$; HRMS (ES+) $m / z$ for $\mathrm{C}_{26} \mathrm{H}_{34} \mathrm{O}_{3} \mathrm{SiNa}[\mathrm{M}+\mathrm{Na}]^{+}$calcd 445.2175, found 445.2176 .

\section{1-[(1R,2S,3R)-3-(Dimethyl-phenyl-silanyl)-1-((R)-1-methyl-but-3-ynyl)-2-triethylsilanyloxy- pent-4-enyloxymethyl]-4-methoxy-benzene (7)}

To a solution of $21(1.01 \mathrm{~g}, 2.39 \mathrm{mmol})$ in DMF $(2.5 \mathrm{~mL})$ was added imidazole $(0.50 \mathrm{~g}, 7.4$ mmol) and triethylsilylchloride $(1.21 \mathrm{~mL}, 7.17 \mathrm{mmol})$. The reaction was heated to $45^{\circ} \mathrm{C}$ for $17 \mathrm{~h}$ and then quenched with water $(15 \mathrm{~mL})$ and extracted with $\mathrm{Et}_{2} \mathrm{O}(25 \mathrm{~mL} \times 3)$. The organic phase was washed with brine, dried over anhydrous $\mathrm{MgSO}_{4}$, filtered and concentrated. The crude product was purified by flash column chromatography to afford $7(1.19 \mathrm{~g}, 93 \%)$ as a colorless oil: $[\alpha]^{25} \mathrm{D}=+31^{\circ}\left(c 1.30, \mathrm{CHCl}_{3}\right) ;{ }^{1} \mathrm{H} \mathrm{NMR}\left(400 \mathrm{MHz}, \mathrm{CDCl}_{3}\right) \delta 7.49-7.52(\mathrm{~m}, 2 \mathrm{H})$, 7.29-7.35 (m, 3H), $7.18(\mathrm{~d}, J=8.8 \mathrm{~Hz}, 2 \mathrm{H}), 6.86(\mathrm{~d}, J=8.8 \mathrm{~Hz}, 2 \mathrm{H}), 6.12(\mathrm{dt}, J=10.8,17.2$ $\mathrm{Hz}, 1 \mathrm{H}), 4.91(\mathrm{dd}, J=10.4,2.0 \mathrm{~Hz}, 1 \mathrm{H}), 4.79$ (dd, $J=17.2,1.6 \mathrm{~Hz}, 1 \mathrm{H}), 4.30$ (AB, $J=18.8$ $\mathrm{Hz}, 1 \mathrm{H}), 4.27$ (d, $J=12.4 \mathrm{~Hz}, 1 \mathrm{H}), 4.50-4.70(\mathrm{~m}, 1 \mathrm{H}), 3.81$ (s, 3H), 3.22 (dd, J = 3.6, 8.4 Hz, $1 \mathrm{H}), 2.31-2.36(\mathrm{~m}, 2 \mathrm{H}), 2.19(\mathrm{dt}, J=3.2,16.8 \mathrm{~Hz}, 1 \mathrm{H}), 2.09-2.13(\mathrm{~m}, 1 \mathrm{H}), 1.94$ (app t, $J=2.4$ $\mathrm{Hz}, 1 \mathrm{H}), 1.02$ (d, $J=6.4 \mathrm{~Hz}, 3 \mathrm{H}), 0.91(\mathrm{t}, J=8.4 \mathrm{~Hz}, 9 \mathrm{H}), 0.50-0.57(\mathrm{~m}, 6 \mathrm{H}), 0.34(\mathrm{~s}, 3 \mathrm{H})$, 0.27 (s, 3H); ${ }^{13} \mathrm{C}$ NMR $\left(100 \mathrm{MHz}, \mathrm{CDCl}_{3}\right) \delta 159.1,138.0,136.5,134.3,130.8,129.3,128.9$, 127.6, 113.6, 113.2, 85.3, 83.4, 72.6, 71.5, 69.5, 55.2, 37.0, 32.9, 22.8, 17.0, 7.1, 5.6, -3.2, -4.2; IR (neat) 3309, 2956, 2877, 1613, 1514, $1248 \mathrm{~cm}^{-1}$; HRMS (ES+) $\mathrm{m} / z$ for $\mathrm{C}_{32} \mathrm{H}_{48} \mathrm{O}_{3} \mathrm{Si}_{2} \mathrm{Na}$ $[\mathrm{M}+\mathrm{Na}]^{+}$calcd 559.3040, found 559.3044.

$(4 R, 5 R)-4-((E)-4-\{(2 S, 4 S, 5 R)-4-(D i m e t h y l-p h e n y l-s i l a n y l)-5-[(1 S, 2 R, 3 R)-2-(4-m e t h o x y-$ benzyloxy)-3-methyl-1-triethylsilanyloxy-hex-5-ynyl]-tetrahydro-furan-2-yl\}-but-1-enyl)-2,2dimethyl-5-vinyl-[1,3]dioxolane (24)

A $25-\mathrm{mL}$ round bottom flask was charged with aldehyde 5 (1.06 g, $5.04 \mathrm{mmol})$, allylsilane 7 (8.12 g, $15.1 \mathrm{mmol})$, activated 4 A molecular sieves $(2.0 \mathrm{~g})$ and dichloromethane $(10 \mathrm{~mL})$. The slurry was stirred at room temperature for $10 \mathrm{~min}$ and then cooled to $-78^{\circ} \mathrm{C}$. The cooled reaction was then treated with $\mathrm{BF}_{3} \cdot \mathrm{OEt}_{2}(0.64 \mathrm{~mL}, 5.04 \mathrm{mmol}$, freshly distilled from calcium hydride). The reaction mixture was stirred at $-78^{\circ} \mathrm{C}$ for $21 \mathrm{~h}$ and then quenched with triethylamine $(1 \mathrm{~mL})$. The mixture was diluted with sat. aq. $\mathrm{NaHCO}_{3}(60 \mathrm{~mL})$ and $\mathrm{Et}_{2} \mathrm{O}(50$ $\mathrm{mL})$ and filtered through Celite. The aqueous phase was extracted with $\mathrm{Et}_{2} \mathrm{O}(30 \mathrm{~mL} \times 3)$. The organic phase was washed with brine, dried over anhydrous $\mathrm{MgSO}_{4}$, filtered and concentrated. Purification of the crude product by flash column chromatography afforded $24(1.19 \mathrm{~g}, 48 \%$; (6.27 g of allylsilane 7 was recovered)) as a colorless oil with >20:1 diastereoselectivity: $[\alpha]$ $25_{\mathrm{D}}=+23^{\circ}\left(c 0.76, \mathrm{CHCl}_{3}\right) ;{ }^{1} \mathrm{H} \mathrm{NMR}\left(400 \mathrm{MHz}, \mathrm{CDCl}_{3}\right) \delta 7.47($ app dd, $J=1.6,7.2 \mathrm{~Hz}, 2 \mathrm{H})$, 7.29-7.38 (m, 3H), $7.24(\mathrm{~d}, J=8.4 \mathrm{~Hz}, 2 \mathrm{H}), 6.87(\mathrm{~d}, J=8.4 \mathrm{~Hz}, 2 \mathrm{H}), 5.74-5.82(\mathrm{~m}, 2 \mathrm{H}), 5.41$ (b dd, $J=7.2,15.2 \mathrm{~Hz}, 1 \mathrm{H}), 5.32(\mathrm{~d}, J=17.6 \mathrm{~Hz}, 1 \mathrm{H}), 5.22(\mathrm{~d}, J=10.4 \mathrm{~Hz}, 1 \mathrm{H}), 4.51(\mathrm{~d}, J=$ $10.8 \mathrm{~Hz}, 1 \mathrm{H}), 4.39$ (d, $J=10.8 \mathrm{~Hz}, 1 \mathrm{H}), 4.05$ (app dd, $J=6.8,12.4 \mathrm{~Hz}, 3 \mathrm{H}), 3.81$ (s, 3H), 3.71 $(\mathrm{m}, 1 \mathrm{H}), 3.58(\mathrm{~d}, J=5.6 \mathrm{~Hz}, 1 \mathrm{H}), 3.32(\operatorname{app~t}, J=6.8 \mathrm{~Hz}, 1 \mathrm{H}), 2.03-2.34(\mathrm{~m}, 5 \mathrm{H}), 1.94(\mathrm{t}, J=$ $2.4 \mathrm{~Hz}, 1 \mathrm{H}), 1.79-1.83(\mathrm{~m}, 1 \mathrm{H}), 1.58-1.69(\mathrm{~m}, 3 \mathrm{H}), 1.44(\mathrm{~s}, 3 \mathrm{H}), 1.43(\mathrm{~s}, 3 \mathrm{H}), 1.10(\mathrm{~d}, J=6.8$ $\mathrm{Hz}, 3 \mathrm{H}), 0.95(\mathrm{t}, J=8.0 \mathrm{~Hz}, 9 \mathrm{H}), 0.51-0.61(\mathrm{~m}, 6 \mathrm{H}), 0.32(\mathrm{~s}, 3 \mathrm{H}), 0.32(\mathrm{~s}, 3 \mathrm{H}) ;{ }^{13} \mathrm{C} \mathrm{NMR}$ $\left(100 \mathrm{MHz}, \mathrm{CDCl}_{3}\right) \delta 158.9,137.6,136.4,134.3,133.8,130.9,129.1,128.9,127.8,125.5,118.2$, $113.2,108.6,83.8,83.2,82.1,80.2,78.5,77.2,73.6,73.1,69.2,55.1,35.2,34.5 .34 .3,29.3$, 27.0, 26.9, 26.2, 22.1, 17.1, 7.1, 5.2, -4.1; IR (neat) 3309, 2955, 2250, 2115, 1614, 1514 $\mathrm{cm}^{-1}$; HRMS (ES+) $\mathrm{m} / z$ for $\mathrm{C}_{44} \mathrm{H}_{66} \mathrm{O}_{6} \mathrm{Si}_{2} \mathrm{Na}[\mathrm{M}+\mathrm{Na}]^{+}$calcd 769.4296, found 769.4307. 
$(1 S, 2 R, 3 R)-1-\{(2 S, 5 S)-5-[(E)-4-((4 R, 5 R)-2,2-D i m e t h y l-5-v i n y l-[1,3] d i o x o l a n-4-y l)-b u t-3-e n y l]-$ tetrahydro-furan-2-yl\}-3-methyl-1-triethylsilanyloxy-hex-5-yn-2-ol (25)

To a solution of [3+2] adduct $24(1.81 \mathrm{~g}, 2.42 \mathrm{mmol})$ in DMF $(2.5 \mathrm{~mL})$ was added $\mathrm{TBAF} \cdot 3 \mathrm{H}_{2} \mathrm{O}(3.82 \mathrm{~g}, 12.1 \mathrm{mmol})$. The reaction was fitted with a condenser and placed in a 90 ${ }^{\circ} \mathrm{C}$ oil bath for $72 \mathrm{~h}$. Additional TBAF$\cdot 3 \mathrm{H}_{2} \mathrm{O}(2.0 \mathrm{~g}, 6.34 \mathrm{mmol})$ was added to the reaction three times during the $72 \mathrm{~h}$ period; at hour 8 , hour 32 and hour 56 . After $72 \mathrm{~h}$, the reaction was diluted with $\mathrm{pH} 7$ buffer $(50 \mathrm{~mL})$ and $\mathrm{Et}_{2} \mathrm{O}(30 \mathrm{~mL})$. The aqueous phase was extracted with $\mathrm{Et}_{2} \mathrm{O}(30 \mathrm{~mL} \times 3)$. The organic phase was washed with brine, dried over anhydrous $\mathrm{MgSO}_{4}$, filtered and concentrated. The crude product was purified by flash column chromatography to afford the alcohol product $(1.03 \mathrm{~g}, 85 \%)$ as a colorless oil: $[\alpha]^{25}{ }_{\mathrm{D}}=+6.4^{\circ}(c 0.39$, $\left.\mathrm{CHCl}_{3}\right) ;{ }^{1} \mathrm{H} \mathrm{NMR}\left(400 \mathrm{MHz}, \mathrm{CDCl}_{3}\right) \delta 7.28(\mathrm{~d}, J=8.8 \mathrm{~Hz}, 2 \mathrm{H}), 6.85(\mathrm{~d}, J=8.8 \mathrm{~Hz}, 2 \mathrm{H})$, 5.73-5.81 (m, 2H), 5.41 (app bddd, $J=1.6,6.0,15.6 \mathrm{~Hz}, 1 \mathrm{H}), 5.32(\mathrm{~d}, J=16.4 \mathrm{~Hz}, 1 \mathrm{H}), 5.21$ (dd, $J=1.2,10.4 \mathrm{~Hz}, 1 \mathrm{H}), 4.58($ app q, $J=10.8 \mathrm{~Hz}, 2 \mathrm{H}), 4.03$ (app q, $J=6.8 \mathrm{~Hz}, 2 \mathrm{H}), 3.93$ (app q, $J=7.2 \mathrm{~Hz}, 1 \mathrm{H}$ ), 3.85 (quint., $J=6.4 \mathrm{~Hz}, 1 \mathrm{H}), 3.78(\mathrm{~s}, 3 \mathrm{H}), 3.45-3.50(\mathrm{~m}, 1 \mathrm{H}), 3.35$ $(\mathrm{dd}, J=2.0,8.0 \mathrm{~Hz}, 1 \mathrm{H}), 5.23(\mathrm{bd}, J=6.8 \mathrm{~Hz}, 1 \mathrm{H}), 2.34(\mathrm{ddq}, J=2.4,6.8,16.8 \mathrm{~Hz}, 2 \mathrm{H})$, 2.05-2.24 (m, 3H), $1.99(\mathrm{t}, J=2.4 \mathrm{~Hz}, 1 \mathrm{H}), 1.89-1.95(\mathrm{~m}, 1 \mathrm{H}), 1.78-1.86(\mathrm{~m}, 1 \mathrm{H}), 1.64-1.75$ $(\mathrm{m}, 2 \mathrm{H}), 1.46-1.60(\mathrm{~m}, 2 \mathrm{H}), 1.43(\mathrm{~s}, 3 \mathrm{H}), 1.42(\mathrm{~s}, 3 \mathrm{H}), 1.08(\mathrm{~d}, J=6.8 \mathrm{~Hz}, 3 \mathrm{H}) ;{ }^{13} \mathrm{C} \mathrm{NMR}$ $\left(100 \mathrm{MHz}, \mathrm{CDCl}_{3}\right) \delta 159.2,135.9,134.2,130.4,129.5,125.9,118.4,113.6,108.7,83.1,82.1$, 82.0, 81.6, 80.4, 79.2, 73.5, 73.1, 69.9, 55.1, 35.1, 34.2, 31.0, 29.0, 27.0, 27.0, 21.8, 16.2; IR (neat) 3536, 3296, 2984, 2934, 1613, 1514, $1248 \mathrm{~cm}^{-1}$; HRMS (ES+) $m / z$ for $\mathrm{C}_{30} \mathrm{H}_{42} \mathrm{O}_{6} \mathrm{Na}$ $[\mathrm{M}+\mathrm{Na}]^{+}$calcd 521.2879, found 521.2879.

To a $0{ }^{\circ} \mathrm{C}$ solution of the alcohol from the preceding step $(1.2 \mathrm{~g}, 2.41 \mathrm{mmol})$ and triethylamine $(0.67 \mathrm{~mL}, 4.82 \mathrm{mmol})$ in dichloromethane $(8 \mathrm{~mL})$ was added triethylsilyl trifluoromethanesulfonate $(0.65 \mathrm{~mL}, 2.89 \mathrm{mmol})$. After $5 \mathrm{~min}$ the reaction was quenched with sat. aq. $\mathrm{NaHCO}_{3}(30 \mathrm{~mL})$ and $\mathrm{Et}_{2} \mathrm{O}(30 \mathrm{~mL})$. The aqueous phase was extracted with $\mathrm{Et}_{2} \mathrm{O}(30$ $\mathrm{mL} \times 3$ ). The organic phase was washed with brine, dried over anhydrous $\mathrm{MgSO}_{4}$, filtered and concentrated. The crude product was purified by flash column chromatography to afford the triethylsilyl ether $(1.39 \mathrm{~g}, 94 \%)$ as a colorless oil: $[\alpha]^{25} \mathrm{D}=+11^{\circ}\left(c 0.36, \mathrm{CHCl}_{3}\right) ;{ }^{1} \mathrm{H} \mathrm{NMR}$ $\left(400 \mathrm{MHz}, \mathrm{CDCl}_{3}\right) \delta 7.25(\mathrm{~d}, J=8.8 \mathrm{~Hz}, 2 \mathrm{H}), 6.87(\mathrm{~d}, J=8.8 \mathrm{~Hz}, 2 \mathrm{H}), 5.75-5.85(\mathrm{~m}, 2 \mathrm{H})$, 5.44 (bdd, $J=7.2,15.2 \mathrm{~Hz}, 1 \mathrm{H}$ ), 5.34 (d, $J=17.2 \mathrm{~Hz}, 1 \mathrm{H}), 5.23$ (dd, $J=0.8,10.4 \mathrm{~Hz}, 1 \mathrm{H}$ ), 4.55 (s, 2H), 4.05 (app q, $J=6.4 \mathrm{~Hz}, 2 \mathrm{H}), 3.93($ app q, $J=6.8 \mathrm{~Hz}, 1 \mathrm{H}), 3.80$ (s, 3H), 3.75-3.79 $(\mathrm{m}, 1 \mathrm{H}), 3.74(\mathrm{dd}, J=3.2,6.8 \mathrm{~Hz}, 1 \mathrm{H}), 3.29(\mathrm{dd}, J=3.2,8.8 \mathrm{~Hz}, 1 \mathrm{H}), 2.28-2.40(\mathrm{~m}, 2 \mathrm{H})$, 2.09-2.24 (m, 3H), $1.97(\mathrm{t}, J=2.8 \mathrm{~Hz}, 1 \mathrm{H}), 1.79-1.94(\mathrm{~m}, 2 \mathrm{H}), 1.61-1.70(\mathrm{~m}, 2 \mathrm{H}), 1.51-1.59$ $(\mathrm{m}, 2 \mathrm{H}), 1.45(\mathrm{~s}, 3 \mathrm{H}), 1.44(\mathrm{~s}, 3 \mathrm{H}), 1.10(\mathrm{~d}, J=6.8 \mathrm{~Hz}, 3 \mathrm{H}), 0.96(\mathrm{t}, J=8.0 \mathrm{~Hz}, 9 \mathrm{H}), 0.55-0.72$ $(\mathrm{m}, 6 \mathrm{H}) ;{ }^{13} \mathrm{C} \mathrm{NMR}\left(100 \mathrm{MHz}, \mathrm{CDCl}_{3}\right) \delta 159.0,136.3,134.3,131.0,129.0,125.7,118.4,113.6$, $108.8,83.2,83.1,82.2,82.2,80.4,78.2,75.8,71.9,69.6,55.2,35.3,33.2,31.1,29.2,27.8$, 27.0, 26.9, 22.5, 16.5, 7.0, 5.2; IR (neat) $3308,2954,2875,1612,1514,1247,1057 \mathrm{~cm}^{-1}$; HRMS (ES+) $m / z$ for $\mathrm{C}_{36} \mathrm{H}_{56} \mathrm{O}_{6} \mathrm{SiNa}[\mathrm{M}+\mathrm{Na}]^{+}$calcd 635.3744 , found 635.3754 .

To a $0{ }^{\circ} \mathrm{C}$ solution of the triethylsilyl ether $(0.621 \mathrm{~g}, 1.01 \mathrm{mmol})$ in dichloromethane $(10 \mathrm{~mL})$ and $\mathrm{pH} 7$ buffer $(1 \mathrm{~mL})$ was added DDQ $(0.46 \mathrm{~g}, 2.02 \mathrm{mmol})$. The reaction was stirred for 1 $\mathrm{h}$, and then quenched with sat. aq. $\mathrm{NaHCO}_{3}(40 \mathrm{~mL})$ and $\mathrm{Et}_{2} \mathrm{O}(30 \mathrm{~mL})$. The aqueous phase was extracted with $\mathrm{Et}_{2} \mathrm{O}(30 \mathrm{~mL} \times 3)$. The organic phase was washed with brine, dried over anhydrous $\mathrm{MgSO}_{4}$, filtered and concentrated. Purification of the crude product by flash column chromatography afforded $\mathbf{2 5}(0.49 \mathrm{~g}, 99 \%)$ as a colorless oil: $[\alpha]^{25} \mathrm{D}=+13^{\circ}(c 0.18$, $\mathrm{CHCl}_{3}$ ); ${ }^{1} \mathrm{H}$ NMR (400 MHz, $\left.\mathrm{CDCl}_{3}\right) \delta 5.74-5.83(\mathrm{~m}, 2 \mathrm{H}$ ), 5.43 (app bddd, $J=1.2,6.0,15.2$ $\mathrm{Hz}, 1 \mathrm{H}), 5.33(\mathrm{~d}, J=17.2 \mathrm{~Hz}, 1 \mathrm{H}), 5.23(\mathrm{dd}, J=0.8,10.4 \mathrm{~Hz}, 1 \mathrm{H}), 4.05($ app q, $J=6.4 \mathrm{~Hz}$, $2 \mathrm{H}), 3.76(\mathrm{~m}, 2 \mathrm{H}), 3.67(\mathrm{~d}, J=7.2 \mathrm{~Hz}, 1 \mathrm{H}), 3.18(\mathrm{t}, J=9.6 \mathrm{~Hz}, 1 \mathrm{H}), 2.50(\mathrm{~d}, J=9.6 \mathrm{~Hz}, 1 \mathrm{H})$, $2.48(\mathrm{dt}, J=3.6,16.4 \mathrm{~Hz}, 1 \mathrm{H}), 2.08-2.20(\mathrm{~m}, 2 \mathrm{H}), 1.95(\mathrm{t}, J=2.4 \mathrm{~Hz}, 1 \mathrm{H}), 1.84-1.97(\mathrm{~m}, 2 \mathrm{H})$, $1.74-1.80(\mathrm{~m}, 1 \mathrm{H}), 1.61-1.66(\mathrm{~m}, 1 \mathrm{H}), 1.59-1.46(\mathrm{~m}, 3 \mathrm{H}), 1.44(\mathrm{~s}, 3 \mathrm{H}), 1.43(\mathrm{~s}, 3 \mathrm{H}), 0.99(\mathrm{~d}$, $J=6.8 \mathrm{~Hz}, 3 \mathrm{H}), 0.95(\mathrm{t}, J=8.0 \mathrm{~Hz}, 9 \mathrm{H}), 0.66$ (app dsept. $J=7.6,19.26 \mathrm{H}) ;{ }^{13} \mathrm{CNMR}(100 \mathrm{MHz}$, 
$\left.\mathrm{CDCl}_{3}\right) \delta 136.2,134.3,125.9,118.5,108.8,83.1,82.2,82.2,81.3,78.7,74.5,74.4,69.3,35.7$, $35.2,30.8,29.2,27.4,27.1,27.0,22.1,15.7,7.0,5.3$; IR (neat) 3524, 3310, 2954, 2875, 1379 , $1238,1054 \mathrm{~cm}^{-1}$; HRMS (ES+) $\mathrm{m} / 2$ for $\mathrm{C}_{28} \mathrm{H}_{48} \mathrm{O}_{5} \mathrm{SiNa}[\mathrm{M}+\mathrm{Na}]^{+}$calcd 515.3169 , found 515.3171.

(4R,5S)-4-Methyl-3-((E)-(R)-2-methyl-hexa-3,5-dienoyl)-5-phenyl-oxazolidin-2-one (36)

To a $-78^{\circ} \mathrm{C}$ solution of oxazolidinone $\mathbf{3 5}^{39}(8.75 \mathrm{~g}, 32.2 \mathrm{mmol})$ in THF $(90 \mathrm{~mL})$ was added NaHMDS $(8.28 \mathrm{~g}, 45.1 \mathrm{mmol})$ in THF $(10 \mathrm{~mL})$. The reaction was stirred at $-78{ }^{\circ} \mathrm{C}$ for $1 \mathrm{~h}$ and then treated with methyl trifluoromethanesulfonate $(5.47 \mathrm{~mL}, 48.4 \mathrm{mmol})$. The reaction was quenched after $3 \mathrm{~h}$ with sat. aq. $\mathrm{NH}_{4} \mathrm{Cl}(100 \mathrm{~mL})$ and $\mathrm{Et}_{2} \mathrm{O}(50 \mathrm{~mL})$. The aqueous phase was extracted with $\mathrm{Et}_{2} \mathrm{O}(50 \mathrm{~mL} \times 3)$. The organic phase was washed with brine, dried over anhydrous $\mathrm{MgSO}_{4}$, filtered, and concentrated. Analysis of the crude product by ${ }^{1} \mathrm{H}$ NMR indicated a 5:1 mixture of diastereomers in favor of 36. The crude product was purified by flash column chromatography in $20 \% \mathrm{Et}_{2} \mathrm{O} /$ hexane (minor isomer, $\mathrm{R}_{\mathrm{f}}=0.21$; major isomer, $\left.\mathrm{R}_{\mathrm{f}}=0.36\right)$ to afford $\mathbf{3 6}(7.30 \mathrm{~g}, 79 \%)$ as a colorless oil: $[\alpha]^{25}{ }_{\mathrm{D}}=-27^{\circ}\left(c 0.55, \mathrm{CHCl}_{3}\right) ;{ }^{1} \mathrm{H}$ NMR $\left(400 \mathrm{MHz}, \mathrm{CDCl}_{3}\right) \delta$ 7.35-7.44 (m, 3H), 7.30-7.32 (m, 2H), 6.20-6.37 (m, 2H), $5.83(\mathrm{dd}$, $J=8.4,15.2 \mathrm{~Hz}, 1 \mathrm{H}), 5.66(\mathrm{~d}, J=7.2 \mathrm{~Hz}, 1 \mathrm{H}), 5.21(\operatorname{app~d}, J=17.6 \mathrm{~Hz}, 1 \mathrm{H}), 5.09$ (app d, $J=$ $10.8 \mathrm{~Hz}, 1 \mathrm{H}$ ), 4.53 (quint., $J=7.6 \mathrm{~Hz}, 1 \mathrm{H}$ ), 4.74 (quint., $J=6.8 \mathrm{~Hz}, 1 \mathrm{H}$ ), $1.33(\mathrm{~d}, J=7.2 \mathrm{~Hz}$, $3 \mathrm{H}), 0.90(\mathrm{~d}, J=6.4 \mathrm{~Hz}, 3 \mathrm{H}) ;{ }^{13} \mathrm{C} \mathrm{NMR}\left(100 \mathrm{MHz}, \mathrm{CDCl}_{3}\right) \delta 174.4,152.6,136.5,133.2,132.7$, 132.6, 128.7, 128.7, 125.6, 117.3, 78.8, 55.1, 40.8, 17.3, 14.5; IR (neat) 2981, 2934, 1782, $1699,1356,1197 \mathrm{~cm}^{-1}$; HRMS (ES+) $\mathrm{m} / z$ for $\mathrm{C}_{17} \mathrm{H}_{21} \mathrm{NO}_{4} \mathrm{Na}[\mathrm{M}+\mathrm{Na}]^{+}$calcd 308.1263 , found 308.1262 .

\section{Tricarbonyl[(4R,5S)-4-Methyl-3-((E)-(2S,3R)-2-methyl-hexa-3,5-dienoyl)-5-phenyl- oxazolidin-2-one]iron (38) and Tricarbonyl[(4R,5S)-4-Methyl-3-((E)-(2S,3S)-2-methyl- hexa-3,5-dienoyl)-5-phenyl-oxazolidin-2-one]iron (37)}

To a solution of oxazolidinone 36 ( $2.0 \mathrm{~g}, 7.0 \mathrm{mmol})$ in benzene $(23 \mathrm{~mL})$ was added diiron (nonacarbonyl) (3.8 g, $10.5 \mathrm{mmol})$. The reaction was fitted with a condenser and refluxed for a total of $24 \mathrm{~h}$. Additional diiron(nonacarbonyl) $(1.5 \mathrm{~g}, 4.12 \mathrm{mmol})$ and benzene $(10 \mathrm{~mL})$ was added to the reaction at hour 6 and hour 20. After 24 hours, the reaction was cooled to room temperature, filtered through Celite with an $\mathrm{Et}_{2} \mathrm{O}(25 \mathrm{~mL})$ wash and concentrated to afford a 1:1 mixture of $\mathbf{3 8}$ and $\mathbf{3 7}$. The crude product mixture was separated by flash column chromatography (10\% $\mathrm{Et}_{2} \mathrm{O} /$ hexanes to $40 \% \mathrm{Et}_{2} \mathrm{O} /$ hexanes with 37 (0.78 g) eluting before $38(0.71 \mathrm{~g}), \mathbf{3 8}$ and $\mathbf{3 7}$ combined yield of $50 \%)$.

Data for 38 (yellow solid): $\mathrm{R}_{\mathrm{f}}=0.33\left(30 \% \mathrm{Et}_{2} \mathrm{O} /\right.$ hexane); $[\alpha]^{25}{ }_{\mathrm{D}}=+8^{\circ}\left(c 0.1, \mathrm{CHCl}_{3}\right) ;{ }^{1} \mathrm{H}$ NMR (400MHz, $\left.\mathrm{CDCl}_{3}\right) \delta$ 7.36-7.45 (m, 3H), $7.36($ app d, $J=6.8 \mathrm{~Hz}, 2 \mathrm{H}), 5.71(\mathrm{~d}, J=7.2$ $\mathrm{Hz}, 1 \mathrm{H}$ ), 5.46 (dd, $J=4.8,8.4 \mathrm{~Hz}, 1 \mathrm{H}), 5.22-5.27(\mathrm{~m}, 1 \mathrm{H}), 4.76$ (quint., $J=6.8 \mathrm{~Hz}, 1 \mathrm{H}$ ), $3.82-3.40(\mathrm{~m}, 1 \mathrm{H}), 1.78$ (app dd, $J=1.6,6.8 \mathrm{~Hz}, 1 \mathrm{H}), 1.37$ (d, $J=6.8 \mathrm{~Hz}, 3 \mathrm{H}), 1.15$ (app t, $J$ $=8.8 \mathrm{~Hz}, 1 \mathrm{H}), 0.88(\mathrm{~d}, J=6.4 \mathrm{~Hz}, 3 \mathrm{H}), 0.39(\operatorname{app~dd}, J=2.0,9.6 \mathrm{~Hz}, 1 \mathrm{H}) ;{ }^{13} \mathrm{C} \mathrm{NMR}(100 \mathrm{MHz}$, $\left.\mathrm{CDCl}_{3}\right) \delta 210.8,175.2,152.9,133.2,128.9,128.8,125.7,86.4,81.9,78.9,64.7,55.1,41.0$, 40.4, 19.9, 14.5; IR (neat) 2984, 2047, 1970, 1779, 1697, $1355 \mathrm{~cm}^{-1}$; HRMS (ES+) $\mathrm{m} / \mathrm{z}$ for $\mathrm{C}_{20} \mathrm{H}_{19} \mathrm{FeNO}_{6} \mathrm{Na}[\mathrm{M}+\mathrm{Na}]^{+}$calcd 448.0459 , found 448.0464 .

Data for 37 (yellow solid): $\mathrm{R}_{\mathrm{f}}=0.50\left(30 \% \mathrm{Et}_{2} \mathrm{O} /\right.$ hexane); $[\alpha]^{25} \mathrm{D}=-83^{\circ}\left(c 0.1, \mathrm{CHCl}_{3}\right) ;{ }^{1} \mathrm{H}$ NMR $\left(400 \mathrm{MHz}, \mathrm{CDCl}_{3}\right) \delta 7.30-7.45(\mathrm{~m}, 5 \mathrm{H}), 5.71(\mathrm{~d}, J=7.2 \mathrm{~Hz}, 1 \mathrm{H}), 5.28-5.33(\mathrm{~m}, 2 \mathrm{H})$, 4.84 (quint., $J=6.8 \mathrm{~Hz}, 1 \mathrm{H}), 3.65-3.78(\mathrm{~m}, 1 \mathrm{H}), 1.40(\mathrm{~d}, J=6.8 \mathrm{~Hz}, 3 \mathrm{H}), 1.36$ (app dd, $J=$ 7.6, $10.0 \mathrm{~Hz}, 3 \mathrm{H}), 0.92(\mathrm{~d}, J=6.8 \mathrm{~Hz}, 3 \mathrm{H}), 0.45($ app dd, $J=2.8,8.8 \mathrm{~Hz}, 1 \mathrm{H}) ;{ }^{13} \mathrm{C} \mathrm{NMR}$ $\left(100 \mathrm{MHz}, \mathrm{CDCl}_{3}\right) \delta 211.4,174.6,152.6,133.2,128.7,125.6,87.1,82.7,79.2,62.9,55.4,43.4$, 40.4, 26.3, 22.3, 14.3; IR (neat) 2977, 2046, 1978, 1782, 1700, $1342 \mathrm{~cm}^{-1}$; HRMS (ES+) $\mathrm{m} /$ $z$ for $\mathrm{C}_{20} \mathrm{H}_{19} \mathrm{FeNO}{ }_{6} \mathrm{Na}[\mathrm{M}+\mathrm{Na}]^{+}$calcd 448.0459, found 448.0462. 


\section{TricarbonyI[(E)-(2S,3R)-2-Methyl-hexa-3,5-dienoic acid]iron (9)}

To a $0{ }^{\circ} \mathrm{C}$ solution of oxazolidinone $38(1.15 \mathrm{~g}, 2.70 \mathrm{mmol})$ in THF $(21 \mathrm{~mL})$ and water $(7 \mathrm{~mL})$ was added $\mathrm{LiOH}(0.194 \mathrm{~g}, 8.11 \mathrm{mmol})$. The reaction was stirred for $1.5 \mathrm{~h}$ and then quenched with $1 \mathrm{M} \mathrm{HCl}(25 \mathrm{~mL})$ and $\mathrm{Et}_{2} \mathrm{O}(25 \mathrm{~mL})$. The aqueous phase was extracted with $\mathrm{Et}_{2} \mathrm{O}(25 \mathrm{~mL}$ $\times 3$ ). The organic phase was dried over anhydrous $\mathrm{MgSO}_{4}$, filtered and concentrated. The crude carboxylic acid was purified by flash column chromatography to afford $\mathbf{9}(0.423 \mathrm{~g}, 58 \%)$ as a yellow solid: $[\alpha]^{25}{ }_{\mathrm{D}}=+11^{\circ}\left(c 0.1, \mathrm{CHCl}_{3}\right) ;{ }^{1} \mathrm{H} \mathrm{NMR}\left(400 \mathrm{MHz}, \mathrm{CDCl}_{3}\right) \delta 10.40(\mathrm{bs}, 1 \mathrm{H})$, 5.38-5.44 (m, 1H), 5.25-5.30 (m, 1H), $2.32(\mathrm{bs}, 1 \mathrm{H}), 1.81(\mathrm{~d}, J=6.4 \mathrm{~Hz}, 1 \mathrm{H}), 1.35(\mathrm{~d}, J=6.4$ $\mathrm{Hz}, 3 \mathrm{H}), 0.94($ app t, $J=9.2 \mathrm{~Hz}, 1 \mathrm{H}), 0.38(\mathrm{~d}, J=7.6 \mathrm{~Hz}, 1 \mathrm{H}) ;{ }^{13} \mathrm{C} \mathrm{NMR}\left(100 \mathrm{MHz}, \mathrm{CDCl}_{3}\right)$ $\delta$ 211.1, 181.1, 87.1, 82.3, 63.1, 44.0, 40.5, 19.0; IR (neat) 2983, 2049, 1971, $1705 \mathrm{~cm}^{-1}$; HRMS (EI+) $m / z$ for $\mathrm{C}_{9} \mathrm{H}_{10} \mathrm{FeO}_{4}[\mathrm{M}-\mathrm{CO}]^{+}$calcd 237.9928, found 237.9918. The spectroscopic data obtained for 9 were fully consistent with data for racemic 9 previously published by Donaldson. ${ }^{40}$

\section{TricarbonyI[(E)-(2S,3S)-2-Methyl-hexa-3,5-dienoic acid]iron (10)}

To a room temperature solution of oxazolidinone $37(1.08 \mathrm{~g}, 2.54 \mathrm{mmol})$ in THF $(18 \mathrm{~mL})$ and water $(6 \mathrm{~mL})$ was added $\mathrm{LiOH}(0.304 \mathrm{~g}, 12.7 \mathrm{mmol})$. The reaction was stirred for $6.5 \mathrm{~h}$ and then quenched with $1 \mathrm{M} \mathrm{HCl}(25 \mathrm{~mL})$ and $\mathrm{Et}_{2} \mathrm{O}(25 \mathrm{~mL})$. The aqueous phase was extracted with $\mathrm{Et}_{2} \mathrm{O}(25 \mathrm{~mL} \times 3)$. The organic phase was dried over anhydrous $\mathrm{MgSO}_{4}$, filtered and concentrated. The crude carboxylic acid was purified by flash column chromatography to afford $10(0.421 \mathrm{~g}, 62 \%)$ as a yellow solid: $[\alpha]^{25} \mathrm{D}=-140^{\circ}\left(c 0.39, \mathrm{CHCl}_{3}\right) ;{ }^{1} \mathrm{HNMR}(400 \mathrm{MHz}$, $\left.\mathrm{CDCl}_{3}\right) \delta 11.26(\mathrm{bs}, 1 \mathrm{H}), 5.22-5.30(\mathrm{~m}, 2 \mathrm{H}), 2.37-2.45(\mathrm{~m}, 1 \mathrm{H}), 1.74-1.78(\mathrm{~m}, 1 \mathrm{H}), 1.38(\mathrm{~d}$, $J=6.8 \mathrm{~Hz}, 3 \mathrm{H}), 1.05($ app dd, $J=8.0,10.0 \mathrm{~Hz}, 1 \mathrm{H}), 0.36(\operatorname{app~dd}, J=4.4,8.8 \mathrm{~Hz}, 1 \mathrm{H}) ;{ }^{13} \mathrm{C}$ NMR $\left(100 \mathrm{MHz}, \mathrm{CDCl}_{3}\right) \delta 211.0,181.0,86.4,82.2,62.1,45.2,39.8,21.4$; IR (neat) 2979, 2934, 2049, 1974,1708 $\mathrm{cm}^{-1}$; HRMS (EI+) $\mathrm{m} / z$ for $\mathrm{C}_{9} \mathrm{H}_{10} \mathrm{FeO}_{4}[\mathrm{M}-\mathrm{CO}]^{+}$calcd 237.9928, found 237.9924.

\section{$(E)-(R)-2-M e t h y l-h e x a-3,5-d i e n o i c ~ a c i d ~(13)$}

To a $0{ }^{\circ} \mathrm{C}$ solution of oxazolidinone $36(0.536 \mathrm{~g}, 1.88 \mathrm{mmol})$ and $30 \%(\mathrm{w} / \mathrm{w}) \mathrm{H}_{2} \mathrm{O}_{2}(2.3 \mathrm{~mL}$, $22.6 \mathrm{mmol})$ in THF $(6.3 \mathrm{~mL})$ was added $\mathrm{LiOH}(0.24 \mathrm{~g}, 5.6 \mathrm{mmol})$. The reaction was stirred for $1.5 \mathrm{~h}$ and then quenched with $1 \mathrm{M} \mathrm{HCl}(20 \mathrm{~mL})$ and $\mathrm{Et}_{2} \mathrm{O}(20 \mathrm{~mL})$. The aqueous phase was extracted with $\mathrm{Et}_{2} \mathrm{O}(20 \mathrm{~mL} \times 3)$. The organic phase was washed with brine, dried over anhydrous $\mathrm{MgSO}_{4}$, filtered and concentrated. The crude product was purified by flash column chromatography to afford $\mathbf{1 3}(0.151 \mathrm{~g}, 64 \%)$ : $[\alpha]^{25} \mathrm{D}=-47^{\circ}\left(c 1.05, \mathrm{CHCl}_{3}\right) ;{ }^{1} \mathrm{H}$ NMR (500 $\left.\mathrm{MHz}, \mathrm{CDCl}_{3}\right) \delta 11.07$ (bs, $\left.1 \mathrm{H}\right), 6.34(\mathrm{dt}, J=10.5,17.0 \mathrm{~Hz}, 1 \mathrm{H}), 6.17$ (dd, $J=10.5,15.0 \mathrm{~Hz}$, $1 \mathrm{H}), 5.77(\mathrm{dd}, J=8.0,15.5 \mathrm{~Hz}, 1 \mathrm{H}), 5.20(\mathrm{~d}, J=18.0 \mathrm{~Hz}, 1 \mathrm{H}), 5.09(\mathrm{~d}, J=10.0 \mathrm{~Hz}, 1 \mathrm{H}), 3.22$ (quint., $J=7.5 \mathrm{~Hz}, 1 \mathrm{H}), 1.32(\mathrm{~d}, J=7.0 \mathrm{~Hz}, 3 \mathrm{H}), ;{ }^{13} \mathrm{C} \mathrm{NMR}\left(100 \mathrm{MHz}, \mathrm{CDCl}_{3}\right) \delta 181.1,136.4$, 132.6, 132.0, 118.1, 117.4, 42.6, 17.0; IR (neat) 3088, 2980, 1709, 1414, 1212, $1003 \mathrm{~cm}^{-1}$; HRMS (ESI-TOF) $m / z$ for $\mathrm{C}_{7} \mathrm{H}_{10} \mathrm{NO}_{2} \mathrm{Na}[\mathrm{M}-\mathrm{H}]^{-}$calcd 125.0608, found 125.0607.

\section{2-epi-Amphidinolide $E(2)$ series}

TricarbonyI[(E)-(2R,3S)-2-Methyl-hexa-3,5-dienoic acid (1R,2R)-1-((R)-\{(2S,5S)-5-[(E)-4-((4R, $5 R$ )-2,2-dimethyl-5-vinyl-[1,3]dioxolan-4-yl)-but-3-enyl]-tetrahydro-furan-2-yl\}triethylsilanyloxy-methyl)-2-methyl-pent-4-ynyl ester]iron (39)

To $0{ }^{\circ} \mathrm{C}$ solution of $25(0.435 \mathrm{~g}, 0.883 \mathrm{mmol}), 10(0.332 \mathrm{~g}, 1.24 \mathrm{mmol})$, triethylamine $(0.37$ $\mathrm{mL}, 2.65 \mathrm{mmol})$, and DMAP (0.108 $\mathrm{g}, 0.883 \mathrm{mmol})$ in THF $(1.8 \mathrm{~mL})$ was added 2,4,6trichlorobenzoyl chloride $(0.19 \mathrm{~mL}, 1.24 \mathrm{mmol})$. The reddish brown solution was stirred at 0 ${ }^{\circ} \mathrm{C}$ for $1 \mathrm{~h}$ and allowed to warm to room temperature over another $1 \mathrm{~h}$. After complete consumption of 25 was observed by TLC analysis, the reaction was quenched with sat. aq. $\mathrm{NaHCO}_{3}(30 \mathrm{~mL})$ and $\mathrm{Et}_{2} \mathrm{O}(30 \mathrm{~mL})$. The aqueous phase was extracted with $\mathrm{Et}_{2} \mathrm{O}(25 \mathrm{~mL} \times$ 
3). The organic phase was washed with sat. aq. $\mathrm{NH}_{4} \mathrm{Cl}$, brine, dried over anhydrous $\mathrm{MgSO}_{4}$, filtered and concentrated. Purification of the crude product by flash column chromatography afforded $39(0.614 \mathrm{~g}, 94 \%)$ as a colorless oil: $[\alpha]^{25} \mathrm{D}=-3.8^{\circ}\left(c 0.87, \mathrm{CHCl}_{3}\right) ;{ }^{1} \mathrm{H} \mathrm{NMR}$ $\left(400 \mathrm{MHz}, \mathrm{CDCl}_{3}\right) \delta 5.75-5.85(\mathrm{~m}, 2 \mathrm{H}), 5.40-5.48(\mathrm{~m}, 1 \mathrm{H}), 5.35-5.39(\mathrm{~m}, 1 \mathrm{H}), 5.33(\mathrm{~d}, J=$ $16.4 \mathrm{~Hz}, 1 \mathrm{H}), 5.22-5.29(\mathrm{~m}, 1 \mathrm{H}), 5.24(\mathrm{~d}, J=10.4 \mathrm{~Hz}, 1 \mathrm{H}), 4.72(\mathrm{~d}, J=8.8 \mathrm{~Hz}, 1 \mathrm{H}), 4.03-4.08$ $(\mathrm{m}, 2 \mathrm{H}), 3.67-3.78(\mathrm{~m}, 1 \mathrm{H}), 3.69(\mathrm{dd}, J=2.0,7.2 \mathrm{~Hz}, 1 \mathrm{H}), 3.58($ app q, $J=6.8 \mathrm{~Hz}, 1 \mathrm{H})$, 2.00-2.34 (m, 7H), $1.96(\mathrm{t}, J=2.4 \mathrm{~Hz}, 1 \mathrm{H}), 1.77-1.94(\mathrm{~m}, 3 \mathrm{H}), 1.59-1.69(\mathrm{~m}, 1 \mathrm{H}), 1.50-1.59$ $(\mathrm{m}, 1 \mathrm{H}), 1.40-1.50(\mathrm{~m}, 1 \mathrm{H}), 1.44(\mathrm{~s}, 3 \mathrm{H}), 1.44(\mathrm{~s}, 3 \mathrm{H}), 1.34(\mathrm{~d}, J=6.8 \mathrm{~Hz}, 3 \mathrm{H}), 1.09$ (d, $J=$ $6.8 \mathrm{~Hz}, 3 \mathrm{H}), 0.92-1.02(\mathrm{~m}, 1 \mathrm{H}), 0.96(\mathrm{t}, J=7.6 \mathrm{~Hz}, 9 \mathrm{H}), 0.57-0.73(\mathrm{~m}, 6 \mathrm{H}), 0.35$ (b d, $J=9.2$ $\mathrm{Hz}, 1 \mathrm{H}) ;{ }^{13} \mathrm{C} \mathrm{NMR}\left(100 \mathrm{MHz}, \mathrm{CDCl}_{3}\right) \delta 211.1,173.5,135.9,134.2,125.9,118.2,108.7,87.0$, 82.2, 82.1, 82.1, 80.4, 78.4, 77.3, 75.1, 69.7, 63.8, 44.5, 40.3, 35.1, 33.3, 30.8, 29.1, 27.6, 27.0, 27.0, 22.0, 19.3, 16.1, 6.9, 5.3; IR (neat) 3311, 2954, 2050, 1980, 1732, $1237 \mathrm{~cm}^{-1}$; HRMS (ES+) $\mathrm{m} / 2$ for $\mathrm{C}_{38} \mathrm{H}_{56} \mathrm{FeO}_{9} \mathrm{SiNa}[\mathrm{M}+\mathrm{Na}]^{+}$calcd 763.2941, found 763.2955.

\section{(E)-(S)-2-Methyl-hexa-3,5-dienoic acid (1R,2R)-1-((R)-\{(2S,5S)-5-[(E)-4-((4R,5R)-2,2- dimethyl-5-vinyl-[1,3]dioxolan-4-yl)-but-3-enyl]-tetrahydro-furan-2-yl\}-triethylsilanyloxy- methyl)-2-methyl-pent-4-ynyl ester (73)}

To a $0{ }^{\circ} \mathrm{C}$ solution of $39(0.574 \mathrm{~g}, 0.775 \mathrm{mmol})$ in acetone $(8 \mathrm{~mL})$ was added cerium ammonium nitrate (CAN) $(0.935 \mathrm{~g}, 1.70 \mathrm{mmol})$. The reaction was stirred for $1.5 \mathrm{~h}$, then quenched with triethylamine $(2 \mathrm{~mL})$ and diluted with sat. aq. $\mathrm{NaHCO}_{3}(50 \mathrm{~mL})$ and $\mathrm{Et}_{2} \mathrm{O}(50 \mathrm{~mL})$. The aqueous phase was extracted with $\mathrm{Et}_{2} \mathrm{O}(30 \mathrm{~mL} \times 3)$. The organic phase was washed with brine, dried over anhydrous $\mathrm{MgSO}_{4}$, filtered and concentrated. The crude product was purified by flash column chromatography to afford $73(0.447 \mathrm{~g}, 96 \%)$ as a colorless oil: $[\alpha]^{25} \mathrm{D}=+22^{\circ}(c 0.98$, $\left.\mathrm{CHCl}_{3}\right) ;{ }^{1} \mathrm{H}$ NMR $\left(400 \mathrm{MHz}, \mathrm{CDCl}_{3}\right) \delta 6.31(\mathrm{dt}, J=10.0,17.2,1 \mathrm{H}), 6.15(\mathrm{dd}, J=10.4,15.2$ $\mathrm{Hz}, 1 \mathrm{H}), 5.72-5.85(\mathrm{~m}, 3 \mathrm{H}), 5.40-5.49(\mathrm{~m}, 1 \mathrm{H}), 5.33(\mathrm{~d}, J=16.4 \mathrm{~Hz}, 1 \mathrm{H}), 5.23(\mathrm{~d}, J=10.0$ $\mathrm{Hz}, 1 \mathrm{H}), 5.18(\mathrm{~d}, J=16.2,1 \mathrm{H}), 5.07(\mathrm{~d}, J=11.2 \mathrm{~Hz}, 1 \mathrm{H}), 4.73(\operatorname{app~dd}, J=2.4,8.8 \mathrm{~Hz}, 1 \mathrm{H})$, 4.02-4.08 (m, 2H), 3.57-3.77 (m, 3H), 3.21 (quint., $J=7.2 \mathrm{~Hz}, 1 \mathrm{H}), 1.96-2.26(\mathrm{~m}, 5 \mathrm{H}), 1.94$ $(\mathrm{t}, J=2.4 \mathrm{~Hz}, 1 \mathrm{H}), 1.77-1.98(\mathrm{~m}, 2 \mathrm{H}), 1.58-1.68(\mathrm{~m}, 1 \mathrm{H}), 1.37-1.58(\mathrm{~m}, 3 \mathrm{H}), 1.44(\mathrm{~s}, 3 \mathrm{H}), 1.43$ $(\mathrm{s}, 3 \mathrm{H}), 1.30(\mathrm{~d}, J=7.2 \mathrm{~Hz}, 3 \mathrm{H}), 1.08(\mathrm{~d}, J=6.8 \mathrm{~Hz}, 3 \mathrm{H}), 0.96(\mathrm{t}, J=8.0 \mathrm{~Hz}, 9 \mathrm{H}), 0.57-0.73$ $(\mathrm{m}, 6 \mathrm{H}) ;{ }^{13} \mathrm{C} \mathrm{NMR}\left(100 \mathrm{MHz}, \mathrm{CDCl}_{3}\right) \delta 173.4,136.2,135.8,134.2,132.3,132.1,125.8,118.1$, 116.8, 108.6, 82.1, 82.1, 82.0, 80.3, 78.3, 77.2, 75.1, 69.6, 42.9, 35.0, 33.2, 30.8, 29.0, 27.5, 26.9, 26.8, 21.8, 17.0, 16.0, 6.8, 5.2; IR (neat) 3311, 2953, 2876, 2050, 1983, 1738, 1732, 1240 $\mathrm{cm}^{-1}$; HRMS (ES+) $\mathrm{m} / z$ for $\mathrm{C}_{35} \mathrm{H}_{56} \mathrm{O}_{6} \mathrm{SiNa}[\mathrm{M}+\mathrm{Na}]^{+}$calcd 623.3744, found 623.3737.

\section{$(4 E, 11 E, 13 E)-(1 S, 6 R, 10 R, 15 S, 18 R, 19 R, 20 S)-8,8,15-T r i m e t h y l-18-((R)-1-m e t h y l-b u t-3-$ ynyl)-19-triethylsilanyloxy-7,9,17,23-tetraoxa-tricyclo[18.2.1.06,10]tricosa-4,11,13-trien-16- one (40)}

To a solution of polyene $73(50 \mathrm{mg}, 0.083 \mathrm{mmol})$ in dichloromethane $(83 \mathrm{~mL})$ was added Grubbs' first generation catalyst ( $14 \mathrm{mg}, 0.017 \mathrm{mmol})$ in dichloromethane $(2 \mathrm{~mL})$. The reaction was fitted with a condenser, refluxed for $18 \mathrm{~h}$ and condensed. The crude product was purified by flash column chromatography to afford $40(29 \mathrm{mg}, 60 \%)$ as a colorless oil. In addition, an inseparable mixture of products thought to arrive by enyne metathesis $(7 \mathrm{mg}, 15 \%)$ was also isolated. Spectroscopic data for 40: $[\alpha]^{25} \mathrm{D}=-57^{\circ}\left(c 0.57, \mathrm{CHCl}_{3}\right) ;{ }^{1} \mathrm{H} \mathrm{NMR}(400 \mathrm{MHz}$, $\left.\mathrm{CDCl}_{3}\right) \delta 6.15-6.26(\mathrm{~m}, 2 \mathrm{H}), 5.93(\mathrm{dd}, J=4.8,14.8 \mathrm{~Hz}, 1 \mathrm{H}), 5.71(\mathrm{ddd}, J=3.6,9.6,15.2 \mathrm{~Hz}$, $1 \mathrm{H}), 5.56(\mathrm{dd}, J=8.4,14.0 \mathrm{~Hz}, 1 \mathrm{H}), 5.34(\mathrm{dd}, J=8.0,14.8 \mathrm{~Hz}, 1 \mathrm{H}), 4.55(\mathrm{~d}, J=9.2 \mathrm{~Hz}, 1 \mathrm{H})$, $4.03(\mathrm{dt}, J=8.8,21.2 \mathrm{~Hz}, 2 \mathrm{H}), 3.68(\mathrm{~d}, J=8.4 \mathrm{~Hz}, 1 \mathrm{H}), 3.28-3.37(\mathrm{~m}, 1 \mathrm{H}), 3.18-3.28(\mathrm{~m}, 2 \mathrm{H})$, 2.19-2.36 (m, 3H), 1.82-2.03 (m, 3H), 1.62-1.73 (m, 1H), 1.40-1.55 (m, 2H), $1.43(\mathrm{~s}, 6 \mathrm{H}), 1.31$ $(\mathrm{d}, J=6.8 \mathrm{~Hz}, 3 \mathrm{H}), 1.13-1.28(\mathrm{~m}, 3 \mathrm{H}), 1.06(\mathrm{~d}, J=6.8 \mathrm{~Hz}, 3 \mathrm{H}), 0.95(\mathrm{t}, J=8.0 \mathrm{~Hz}, 9 \mathrm{H})$, $0.53-0.70(\mathrm{~m}, 6 \mathrm{H}) ;{ }^{13} \mathrm{C} \mathrm{NMR}\left(100 \mathrm{MHz}, \mathrm{CDCl}_{3}\right) \delta 172.5,138.1,135.9,134.6,128.9,127.5$, $125.9,109.0,83.1,82.8,82.2,79.9,78.5,77.2,75.0,69.4,43.3,33.1,32.1,29.6,28.5,27.2$, 27.1, 27.1, 22.3, 15.5, 12.0, 7.1, 5.6; IR (neat) 3310, 2935, 2874, 1726, 1238, $1052 \mathrm{~cm}^{-1}$; HRMS (ES+) $\mathrm{m} / z$ for $\mathrm{C}_{33} \mathrm{H}_{52} \mathrm{O}_{6} \mathrm{SiNa}[\mathrm{M}+\mathrm{Na}]^{+}$calcd 595.3431, found 595.3438. 
$(4 E, 11 E, 13 E)-(1 S, 6 R, 10 R, 15 S, 18 R, 19 R, 20 S)-18-((R)-3-l o d o-1-m e t h y l-b u t-3-e n y l)-8,8,15-$ trimethyl-19-triethylsilanyloxy-7,9,17,23-tetraoxa-tricyclo[18.2.1.06,10] tricosa-4,11,13trien-16-one (41)

To a $0{ }^{\circ} \mathrm{C}$ solution of $i$ - $\mathrm{Pr}_{2} \mathrm{NH}(0.45 \mathrm{~mL}, 3.2 \mathrm{mmol})$ in THF $(3 \mathrm{~mL})$ was added $n$-BuLi $(1.24$ $\mathrm{mL}$ of a $2.41 \mathrm{M}$ solution in hexanes, $3.0 \mathrm{mmol}$ ). The reaction was allowed to stir for $30 \mathrm{~min}$ and then cooled to $-30{ }^{\circ} \mathrm{C}$. To this mixture was added $\mathrm{Bu}_{3} \mathrm{SnH}(0.80 \mathrm{~mL}, 3.0 \mathrm{mmol})$. After $1 \mathrm{~h}$, $\mathrm{Et}_{2} \mathrm{AlCl}(1.7 \mathrm{~mL}$ of a $1.8 \mathrm{M}$ solution in toluene, $3.0 \mathrm{mmol})$ was added. The reaction was stirred at $-30{ }^{\circ} \mathrm{C}$ for another $1.5 \mathrm{~h}$ and then used immediately in the stannylalumination-protonolysis of 40 .

To a $-30{ }^{\circ} \mathrm{C}$ solution of $\mathbf{4 0}(23 \mathrm{mg}, 0.40 \mathrm{mmol})$ in THF $(1 \mathrm{~mL})$ was added $\mathrm{Bu}_{3} \mathrm{Sn}_{-} \mathrm{AlEt}_{2}(0.57$ $\mathrm{mL}$ of the $0.42 \mathrm{M}$ solution from above, $0.24 \mathrm{mmol})$, followed by CuCN (1 mg, $0.012 \mathrm{mmol}$ ). The bright orange solution was stirred for $1 \mathrm{~h}$ at $-30^{\circ} \mathrm{C}$, then quenched with sat. aq. $\mathrm{NH}_{4} \mathrm{Cl}$ $(20 \mathrm{~mL})$ and $\mathrm{Et}_{2} \mathrm{O}(20 \mathrm{~mL})$. This mixture was stirred vigorously at room temp for $15 \mathrm{~min}$. The aqueous phase was extracted with $\mathrm{Et}_{2} \mathrm{O}(10 \mathrm{~mL} \times 3)$. The organic phase was washed with brine, dried over anhydrous $\mathrm{MgSO}_{4}$, filtered and concentrated. Purification of the crude product by flash column chromatography afforded the vinylstannane product $(18 \mathrm{mg}, 51 \%)$ as a colorless oil: $[\alpha]^{25} \mathrm{D}=-78^{\circ}\left(c 0.12, \mathrm{CHCl}_{3}\right) ;{ }^{1} \mathrm{H}$ NMR $\left(400 \mathrm{MHz}, \mathrm{CDCl}_{3}\right) \delta 6.15-6.28(\mathrm{~m}, 2 \mathrm{H}), 5.96$ $(\mathrm{dd}, J=4.8,14.4 \mathrm{~Hz}, 1 \mathrm{H}), 5.72(\mathrm{ddd}, J=3.2,9.2,15.2 \mathrm{~Hz}, 1 \mathrm{H}), 5.64\left(\right.$ app t,${ }^{3} J_{S n-H}=69.2 \mathrm{~Hz}$, $1 \mathrm{H}), 5.57(\mathrm{dd}, J=8.4,14.0 \mathrm{~Hz}, 1 \mathrm{H}), 5.34(\mathrm{dd}, J=8.4,15.2 \mathrm{~Hz}, 1 \mathrm{H}), 5.16\left(\right.$ app t$^{3}{ }^{3} J_{S n-H}=31.6$ $\mathrm{Hz}, 1 \mathrm{H}), 4.49(\mathrm{~d}, J=10.0 \mathrm{~Hz}, 1 \mathrm{H}), 4.04(\operatorname{app~dt}, J=8.8,20.4 \mathrm{~Hz}, 1 \mathrm{H}), 3.74(\mathrm{~d}, J=8.4 \mathrm{~Hz}$, $1 \mathrm{H}), 3.28-3.36(\mathrm{~m}, 1 \mathrm{H}), 3.18-3.28(\mathrm{~m}, 2 \mathrm{H}), 2.28-2.40(\mathrm{~m}, 3 \mathrm{H}), 1.82-2.13(\mathrm{~m}, 4 \mathrm{H}), 1.62-1.74$ $(\mathrm{m}, 1 \mathrm{H}), 1.40-1.55(\mathrm{qm}, 7 \mathrm{H}), 1.44(\mathrm{~s}, 6 \mathrm{H}), 1.13-1.38(\mathrm{~m}, 11 \mathrm{H}), 0.85-1.00(\mathrm{~m}, 24 \mathrm{H}), 0.79(\mathrm{~d}$, $J=6.4 \mathrm{~Hz}, 3 \mathrm{H}), 0.55-0.70(\mathrm{~m}, 6 \mathrm{H}) ;{ }^{13} \mathrm{C}$ NMR $\left(100 \mathrm{MHz}, \mathrm{CDCl}_{3}\right) \delta 172.6,153.9,138.2,136.0$, 134.9, 131.1, 128.7, 127.3, 127.0, 125.8, 109.0, 83.1, 82.3, 80.1, 79.9, 74.9, 45.3, 43.3, 32.7, 32.1, 29.6, 29.2, 29.1, 29.0, 28.5, 27.4, 27.2, 27.1, 14.8, 13.7, 12.0, 9.5, 7.1, 5.6; IR (neat) 2955, $1727,1378,1239,1052 \mathrm{~cm}^{-1}$; HRMS (ES+) $\mathrm{m} / z$ for $\mathrm{C}_{45} \mathrm{H}_{80} \mathrm{O}_{6} \mathrm{SiSnNa}[\mathrm{M}+\mathrm{Na}]^{+}$calcd 887.4644, found 887.4666 .

To a $-45{ }^{\circ} \mathrm{C}$ solution of the vinylstannane from the preceding step $(20 \mathrm{mg}, 0.023 \mathrm{mmol})$ in dichloromethane $(1 \mathrm{~mL})$ was added NIS $(6 \mathrm{mg}, 0.03 \mathrm{mmol})$. The reaction was stirred at $-45^{\circ}$ $\mathrm{C}$ for $1 \mathrm{~h}$ and then quenched with sat. aq. $\mathrm{Na}_{2} \mathrm{~S}_{2} \mathrm{O}_{3}(30 \mathrm{~mL})$ and extracted with $\mathrm{Et}_{2} \mathrm{O}(20 \mathrm{~mL}$ $\times 3$ ). The organic phase was washed with brine, dried over anhydrous $\mathrm{MgSO}_{4}$, filtered and concentrated. The crude product was purified by flash column chromatography to afford $\mathbf{4 1}$ $(15 \mathrm{mg}, 93 \%)$ as a colorless oil: $[\alpha]^{25} \mathrm{D}=-72^{\circ}\left(c 0.43, \mathrm{CHCl}_{3}\right) ;{ }^{1} \mathrm{H}$ NMR $\left(400 \mathrm{MHz}, \mathrm{CDCl}_{3}\right)$ $\delta$ 6.17-6.27 (m, 2H), $6.02(\mathrm{~s}, 1 \mathrm{H}), 5.93(\mathrm{dd}, J=5.2,14.4 \mathrm{~Hz}, 1 \mathrm{H}), 5.73$ (ddd, $J=3.6,9.6,15.2$ $\mathrm{Hz}, 1 \mathrm{H}), 5.72(\mathrm{~s}, 1 \mathrm{H}), 5.58(\mathrm{dd}, J=8.8,14.0 \mathrm{~Hz}, 1 \mathrm{H}), 5.34(\mathrm{dd}, J=8.4,15.2 \mathrm{~Hz}, 1 \mathrm{H}), 4.55(\mathrm{~d}$, $J=9.6 \mathrm{~Hz}, 1 \mathrm{H}), 4.03($ app dt, $J=8.8,23.6 \mathrm{~Hz}, 2 \mathrm{H}), 3.72(\mathrm{~d}, J=8.4 \mathrm{~Hz}, 1 \mathrm{H}), 3.28-3.35(\mathrm{~m}$, $1 \mathrm{H}), 3.19-3.28(\mathrm{~m}, 2 \mathrm{H}), 2.29-2.50(\mathrm{~m}, 3 \mathrm{H}), 1.82-2.05(\mathrm{~m}, 3 \mathrm{H}), 1.65-1.73(\mathrm{~m}, 1 \mathrm{H}), 1.45-1.57$ $(\mathrm{m}, 1 \mathrm{H}), 1.43(\mathrm{~s}, 6 \mathrm{H}), 1.33(\mathrm{~d}, J=6.8 \mathrm{~Hz}, 3 \mathrm{H}), 1.12-1.27(\mathrm{~m}, 3 \mathrm{H}), 0.98(\mathrm{t}, J=8.0 \mathrm{~Hz}, 9 \mathrm{H})$, $0.86(\mathrm{~d}, J=6.4 \mathrm{~Hz}, 3 \mathrm{H}), 0.56-0.72(\mathrm{~m}, 6 \mathrm{H}) ;{ }^{13} \mathrm{C} \mathrm{NMR}\left(100 \mathrm{MHz}, \mathrm{CDCl}_{3}\right) \delta 172.8,138.1,136.0$, 134.5, 129.0, 127.5, 127.0, 125.8, 111.5, 109.0, 83.1, 82.2, 80.0, 78.6, 74.9, 48.3, 43.3, 32.8, 32.0, 29.6, 28.5, 27.2, 27.1, 27.1, 14.6, 12.0, 7.2, 5.7; IR (neat) 2933, 2873, 1723, 1239, 1052 $\mathrm{cm}^{-1}$; HRMS (ES+) $\mathrm{m} / \mathrm{z}$ for $\mathrm{C}_{33} \mathrm{H}_{53} \mathrm{IO}_{6} \mathrm{SiNa}[\mathrm{M}+\mathrm{Na}]^{+}$calcd 723.2554 , found 723.25563

\section{2-epi-amphidinolide $E$ (2)}

A solution of vinyl iodide 41 (44 mg, $0.063 \mathrm{mmol}$ ) in a mixture of AcOH, THF and water (4/1/1 ratio) $(1.5 \mathrm{~mL})$ was heated to $40{ }^{\circ} \mathrm{C}$ for $7 \mathrm{~h}$. The mixture was then carefully poured into a separatory funnel containing $\mathrm{Et}_{2} \mathrm{O}(40 \mathrm{~mL})$ and sat. aq. $\mathrm{NaHCO}_{3}(60 \mathrm{~mL})$. The $\mathrm{pH}$ of the aqueous layer was adjusted to $\sim 7$ using solid $\mathrm{NaHCO}_{3}$. The aqueous phase was extracted with $\mathrm{Et}_{2} \mathrm{O}(20 \mathrm{~mL} \times 3)$. The organic phase was washed with brine, dried over anhydrous $\mathrm{MgSO}_{4}$, 
filtered and concentrated. Analysis of the crude product by ${ }^{1} \mathrm{H}$ NMR indicated a 10:1 mixture of the desired $\mathrm{C}(18)$ and undesired $\mathrm{C}(17)$ lactones.

To the crude mixure of vinyl iodide-containing lactones (from above) and $\mathrm{CuCl}$ ( $23 \mathrm{mg}, 0.23$ $\mathrm{mmol})$ in THF $(0.5 \mathrm{~mL})$ was added vinylstannane $6^{12}(78 \mathrm{mg}, 0.21 \mathrm{mmol})$, followed by Pd $\left(\mathrm{PPh}_{3}\right)_{4}(10 \mathrm{mg}, 0.0084 \mathrm{mmol})$ in THF $(0.5 \mathrm{~mL})$. The reaction was stirred at room temperature for $26 \mathrm{~h}$, and then diluted with $\mathrm{Et}_{2} \mathrm{O}(30 \mathrm{~mL})$, filtered through Celite and concentrated. The crude product was purified by flash column chromatography using $10 \%$ methanol/chloroform to afford material that was still contaminated with an organotin impurity. The stannane impurity was removed by HPLC purification with $100 \%$ ethyl acetate eluent on a normal phase, Varian Dynamax Microsorb 60-8 Si, $250 \times 21.4 \mathrm{~mm}$ column. The retention time for 2-epiamphidinolide $\mathrm{E}$ was $8 \mathrm{~min}$. The flow rate was $18 \mathrm{~mL} / \mathrm{min}$. 2-epi-amphidinolide $\mathrm{E}$ was detected using UV absorption ( $\lambda=254 \mathrm{~nm}$ and $280 \mathrm{~nm}$ ) and RI detection. Using the above conditions $11 \mathrm{mg}$ (34\% from 41) of pure 2-epi-amphidinolide $\mathrm{E}(2)$ was isolated: $[\alpha]^{25} \mathrm{D}=-80^{\circ}(c 0.15$, $\left.\mathrm{CHCl}_{3}\right) ;{ }^{1} \mathrm{H}$ NMR $\left(400 \mathrm{MHz}, \mathrm{CDCl}_{3}\right) \delta$ 6.14-6.25 (m, 2H), $6.04(\mathrm{~d}, J=16.0 \mathrm{~Hz}, 1 \mathrm{H}), 5.95-6.04$ $(\mathrm{m}, 1 \mathrm{H}), 5.54-5.74(\mathrm{~m}, 3 \mathrm{H}), 5.30(\mathrm{dd}, J=7.2,15.2 \mathrm{~Hz}, 1 \mathrm{H}), 4.98(\mathrm{~s}, 1 \mathrm{H}), 4.86(\mathrm{~s}, 1 \mathrm{H}), 4.74(\mathrm{~s}$, $1 \mathrm{H}), 4.70(\mathrm{~s}, 1 \mathrm{H}), 4.66$ (d, $J=9.6 \mathrm{~Hz}, 1 \mathrm{H}), 3.95$ (app dt, $J=8.4,19.2 \mathrm{~Hz}, 2 \mathrm{H}), 3.69$ (app t, $J$ $=5.2 \mathrm{~Hz}, 1 \mathrm{H}$ ), 3.46-3.53 (m, 1H), 3.40-3.46 (m, 1H), 3.35 (quint., $J=5.2 \mathrm{~Hz}, 1 \mathrm{H}$ ), $2.72-2.84$ $(\mathrm{m}, 2 \mathrm{H}), 2.22-2.48(\mathrm{~m}, 5 \mathrm{H}), 1.60-1.95(\mathrm{~m}, 5 \mathrm{H}), 1.72(\mathrm{~s}, 3 \mathrm{H}), 1.25-1.52(\mathrm{~m}, 4 \mathrm{H}), 1.34(\mathrm{~d}, J=$ $7.2 \mathrm{~Hz}, 3 \mathrm{H}), 0.91(\mathrm{~d}, J=6.8 \mathrm{~Hz}, 3 \mathrm{H}) ;{ }^{13} \mathrm{C}$ NMR $\left(100 \mathrm{MHz}, \mathrm{CDCl}_{3}\right) \delta 172.9,144.7,144.1$, 134.8, 134.2, 134.1, 133.4, 131.2, 129.7, 129.6, 128.2, 116.1, 110.9, 79.9, 78.7, 77.9, 77.3, 76.6, 73.2, 43.1, 41.5, 36.3, 32.8, 32.3, 30.2, 29.1, 27.5, 22.7, 15.5, 13.4; IR (neat) 3413, 2933, 1723, 1454, 1238, $992 \mathrm{~cm}^{-1}$; HRMS (ES+) $\mathrm{m} / 2$ for $\mathrm{C}_{30} \mathrm{H}_{44} \mathrm{O}_{6} \mathrm{Na}[\mathrm{M}+\mathrm{Na}]^{+}$calcd 523.3036, found 523.3016 .

\section{Synthesis of 19-epi-series Precursors}

\section{(1R,2S)-1-((S)-2,2-Diethyl-[1,3]dioxolan-4-yl)-2-methyl-but-3-en-1-ol (60)}

To a $-78{ }^{\circ} \mathrm{C}$ slurry of L-glyceraldehyde pentylidene ketal $\mathbf{5 8}^{24}(12.1 \mathrm{~g}, 76.5 \mathrm{mmol})$ and $4 \AA$ mol. sieves $(9 \mathrm{~g})$ in toluene $(100 \mathrm{~mL})$ was added $Z-(S, S)$-crotylboronate $\mathbf{5 9}^{23}(153 \mathrm{~mL}$ of a $1.0 \mathrm{M}$ solution in toluene, $153 \mathrm{mmol}$ ). The reaction was stirred at $-78{ }^{\circ} \mathrm{C}$ for $18 \mathrm{~h}$ and then quenched with $2 \mathrm{~N} \mathrm{NaOH}$ aq. $(300 \mathrm{~mL})$. The biphasic mixture was filtered through Celite and extracted with EtOAc $(75 \mathrm{~mL} \times 3)$. The organic phase was washed with brine, dried over anhydrous $\mathrm{MgSO}_{4}$, filtered and concentrated. The crude product was purified by flash column chromatography to afford $\mathbf{6 0}(15.4 \mathrm{~g}, 94 \%)$ as a colorless oil: $[\alpha]^{25} \mathrm{D}=-45^{\circ}(c 4.6$, $\left.\mathrm{CHCl}_{3}\right) ;{ }^{1} \mathrm{H}$ NMR $\left(400 \mathrm{MHz}, \mathrm{CDCl}_{3}\right) \delta 5.71(\mathrm{ddd}, J=8.0,10.0,17.2 \mathrm{~Hz}, 1 \mathrm{H}), 5.04(\mathrm{~d}, J=$ $10.0 \mathrm{~Hz}, 1 \mathrm{H}), 5.00(\mathrm{~s}, 1 \mathrm{H}), 4.00-4.08(\mathrm{~m}, 1 \mathrm{H}), 3.94(\operatorname{app~t}, J=7.6 \mathrm{~Hz}, 1 \mathrm{H}), 3.81$ (app t, $J=7.6$ $\mathrm{Hz}, 1 \mathrm{H}), 3.60-3.66(\mathrm{~m}, 1 \mathrm{H}), 2.18-2.28(\mathrm{~m}, 2 \mathrm{H}), 1.52-1.68(\mathrm{~m}, 4 \mathrm{H}), 1.06(\mathrm{~d}, J=6.4 \mathrm{~Hz}, 3 \mathrm{H})$, 0.80-0.91 (m, 6H); $\left.{ }^{13} \mathrm{C} \mathrm{NMR} \mathrm{(100} \mathrm{MHz,} \mathrm{CDCl}_{3}\right) \delta 140.2,115.4,112.4,76.8,73.8,65.1,40.7$, 29.1, 15.3, 8.2, 7.9; IR (neat) 3481, 2974, 1641, $1463 \mathrm{~cm}^{-1}$; HRMS (ES+) $\mathrm{m} / \mathrm{z}$ for $\mathrm{C}_{12} \mathrm{H}_{22} \mathrm{O}_{3} \mathrm{Na}[\mathrm{M}+\mathrm{Na}]^{+}$calcd 237.1467, found 237.1460.

(3S,4R)-4-((S)-2,2-Diethyl-[1,3]dioxolan-4-yl)-4-(4-methoxy-benzyloxy)-3-methyl-butan-1-ol (61)

Protection of alcohol $\mathbf{6 0}$ as a $p$-methoxybenzyl ether was accomplished using a procedure analogous to that outlined for the conversion of 16 to 17: $\mathrm{NaH}$ ( $3.45 \mathrm{~g}, 144 \mathrm{mmol}), \mathrm{NaI}$ ( 2.7 $\mathrm{g}, 18.0 \mathrm{mmol})$, THF $(200 \mathrm{~mL})$, alcohol $\mathbf{6 0}(15.4 \mathrm{~g}, 71.9 \mathrm{mmol})$ and $p$-methoxybenzyl chloride $(6.38 \mathrm{~mL}, 47.0 \mathrm{mmol})$ were used. The crude product was purified by flash column chromatography to afford the $p$-methoxybenzyl ether $(23.8 \mathrm{~g}, 99 \%)$ as a colorless oil: $[\alpha]^{25} \mathrm{D}$ $=-34^{\circ}\left(c 2.0, \mathrm{CHCl}_{3}\right) ;{ }^{1} \mathrm{H} \mathrm{NMR}\left(400 \mathrm{MHz}, \mathrm{CDCl}_{3}\right) \delta 7.25(\mathrm{~d}, J=9.2 \mathrm{~Hz}, 2 \mathrm{H}), 6.86(\mathrm{~d}, J=$ $9.2 \mathrm{~Hz}, 2 \mathrm{H}), 5.88$ (ddd, $J=7.2,10.0,17.2 \mathrm{~Hz}, 1 \mathrm{H}), 5.07$ (d $J=17.2 \mathrm{~Hz}, 1 \mathrm{H}), 5.03(\mathrm{~d}, J=10.4$ $\mathrm{Hz}, 1 \mathrm{H}), 4.61(\mathrm{AB}, J=10.8 \mathrm{~Hz}, 1 \mathrm{H}), 4.55(\mathrm{AB}, J=10.8 \mathrm{~Hz}, 1 \mathrm{H}), 4.12(\mathrm{ddd}, J=6.4,7.6,11.6$ 
$\mathrm{Hz}, 1 \mathrm{H}), 4.00(\mathrm{dd}, J=6.0,7.6 \mathrm{~Hz}, 1 \mathrm{H}), 3.78-3.83(\mathrm{~m}, 1 \mathrm{H}), 3.80(\mathrm{~s}, 3 \mathrm{H}), 3.51(\mathrm{t}, J=5.2 \mathrm{~Hz}$, $1 \mathrm{H}), 2.38-2.48(\mathrm{~m}, 1 \mathrm{H}), 1.55-1.72(\mathrm{~m}, 4 \mathrm{H}), 1.08(\mathrm{~d}, J=6.8 \mathrm{~Hz}, 3 \mathrm{H}), 0.87-0.93(\mathrm{~m}, 6 \mathrm{H}) ;{ }^{13} \mathrm{C}$ NMR $\left(100 \mathrm{MHz}, \mathrm{CDCl}_{3}\right) \delta 159.1,141.3,130.7,129.3,114.4,113.7,112.5,82.4,76.9,74.0$, 66.5, 55.2, 40.2, 29.7, 29.0, 15.0, 8.2, 8.1; IR (neat) 2972, 1614, 1514, $1248 \mathrm{~cm}^{-1}$; HRMS (ES +) $\mathrm{m} / \mathrm{z}$ for $\mathrm{C}_{20} \mathrm{H}_{30} \mathrm{O}_{4} \mathrm{Na}[\mathrm{M}+\mathrm{Na}]^{+}$calcd 357.2042, found 357.2039.

The hydroboration-oxidation of the $p$-methoxybenzyl ether compound was accomplished using a procedure analogous to that outlined for the conversion of $\mathbf{1 6}$ to 17: the $p$-methoxybenzyl ether $(23.8 \mathrm{~g}, 71.2 \mathrm{mmol})$, THF $(50 \mathrm{~mL})$ and 9-BBN $(430 \mathrm{~mL}$ of a $0.5 \mathrm{M}$ THF solution, 136 $\mathrm{mmol}$ ) were used. The crude product was purified by flash column chromatography to afford $61(24.8 \mathrm{~g}, 99 \%)$ as a colorless oil: $[\alpha]^{25} \mathrm{D}=-21^{\circ}\left(c 2.2, \mathrm{CHCl}_{3}\right) ;{ }^{1} \mathrm{H} \mathrm{NMR}(400 \mathrm{MHz}$, $\left.\mathrm{CDCl}_{3}\right) \delta 7.24(\mathrm{~d}, J=8.4 \mathrm{~Hz}, 2 \mathrm{H}), 6.87(\mathrm{~d}, J=8.4 \mathrm{~Hz}, 2 \mathrm{H}), 4.55$ (s, 2H), 4.14 (app q, $J=6.0$ $\mathrm{Hz}, 1 \mathrm{H}), 4.07(\mathrm{dd}, J=5.2,7.6 \mathrm{~Hz}, 1 \mathrm{H}), 3.80(\mathrm{~s}, 3 \mathrm{H}), 3.79($ app q, $J=7.6 \mathrm{~Hz}, 1 \mathrm{H}), 3.69-3.76$ (m, 1H), 3.60-3.69 (m, 1H), 3.48 (dd, $J=2.8,6.4 \mathrm{~Hz}, 1 \mathrm{H}), 1.98-2.06(\mathrm{~m}, 1 \mathrm{H}), 1.72-1.82(\mathrm{~m}$, $1 \mathrm{H}), 1.59-1.70(\mathrm{~m}, 4 \mathrm{H}), 1.50-1.59(\mathrm{~m}, 1 \mathrm{H}), 0.99(\mathrm{~d}, J=6.8 \mathrm{~Hz}, 3 \mathrm{H}), 0.89(\mathrm{q}, J=7.2 \mathrm{~Hz}$, $6 \mathrm{H}) ;{ }^{13} \mathrm{C}$ NMR $\left(100 \mathrm{MHz}, \mathrm{CDCl}_{3}\right) \delta 159.2,130.5,129.3,113.8,112.5,83.1,76.7,73.6,67.7$, 61.2, 55.3, 36.5, 32.5, 29.8, 29.0, 15.2, 8.2, 8.2; IR (neat) 3418, 2934, 2245, $1614 \mathrm{~cm}^{-1}$; HRMS (ES+) $\mathrm{m} / z$ for $\mathrm{C}_{20} \mathrm{H}_{32} \mathrm{O}_{5} \mathrm{Na}[\mathrm{M}+\mathrm{Na}]^{+}$calcd 375.2147 , found 375.2144 .

\section{(S)-2,2-Diethyl-4-[(1R,2S)-1-(4-methoxy-benzyloxy)-2-methyl-pent-4-ynyl]-[1,3]dioxolane (62)}

The oxidation of $\mathbf{6 1}$ was accomplished using a procedure analogous to that outlined for the conversion of $\mathbf{1 7}$ to 18: alcohol $\mathbf{6 1}(24.8 \mathrm{~g}, 70.4 \mathrm{mmol}), \mathrm{CH}_{2} \mathrm{Cl}_{2}(240 \mathrm{~mL})$, DMSO (15.3 mL, $216 \mathrm{mmol}), i$ - $\mathrm{Pr}_{2} \mathrm{NEt}(39 \mathrm{~mL}, 216 \mathrm{mmol})$ and $\mathrm{SO}_{3}$.pyridine $(34 \mathrm{~g}, 216 \mathrm{mmol})$ were used. The crude product was purified by flash column chromatography to afford the aldehyde ( $24.4 \mathrm{~g}$, 99\%) as a colorless oil: $[\alpha]^{25}{ }_{\mathrm{D}}=-18^{\circ}\left(c 1.6, \mathrm{CHCl}_{3}\right) ;{ }^{1} \mathrm{H} \mathrm{NMR}\left(400 \mathrm{MHz}, \mathrm{CDCl}_{3}\right) \delta 9.73$ (s, $1 \mathrm{H}), 7.22(\mathrm{~d}, J=8.4 \mathrm{~Hz}, 2 \mathrm{H}), 6.88(\mathrm{~d}, J=8.8 \mathrm{~Hz}, 2 \mathrm{H}), 4.51(\mathrm{AB}, J=10.8 \mathrm{~Hz}, 1 \mathrm{H}), 4.47$ (AB, $J=11.2 \mathrm{~Hz}, 1 \mathrm{H}), 4.30-4.11(\mathrm{~m}, 2 \mathrm{H}), 3.81(\mathrm{~s}, 3 \mathrm{H}), 3.72-3.80(\mathrm{~m}, 1 \mathrm{H}), 3.45$ (bdd, $J=2.8$, $6.8 \mathrm{~Hz}, 1 \mathrm{H}), 2.45-2.61(\mathrm{~m}, 2 \mathrm{H}), 2.30-2.45(\mathrm{~m}, 1 \mathrm{H}), 1.55-1.70(\mathrm{~m}, 4 \mathrm{H}), 1.03(\mathrm{~d}, J=6.8 \mathrm{~Hz}$, $3 \mathrm{H}), 0.85-0.93(\mathrm{~m}, 6 \mathrm{H}) ;{ }^{13} \mathrm{C}$ NMR $\left(100 \mathrm{MHz}, \mathrm{CDCl}_{3}\right) \delta 202.0,159.2,130.2,129.3,113.7$, 112.6, 82.2, 76.5, 73.2, 67.9, 55.2, 47.630.3, 29.7, 28.9, 15.0, 8.1, 8.1; IR (neat) 2972, 1724, $1514,1249 \mathrm{~cm}^{-1}$; HRMS (ES+) $\mathrm{m} / z$ for $\mathrm{C}_{20} \mathrm{H}_{30} \mathrm{O}_{5} \mathrm{Na}[\mathrm{M}+\mathrm{Na}]^{+}$calcd 373.1991, found 373.1985 .

The Corey-Fuchs homologation of the aldehyde was accomplished using a procedure analogous to that outlined for the conversion of $\mathbf{1 7}$ to $\mathbf{1 8}$ : the aldehyde $(24.4 \mathrm{~g}, 69 \mathrm{mmol})$, $\mathrm{PPh}_{3}(30.9 \mathrm{~g}, 118 \mathrm{mmol}), \mathrm{CH}_{2} \mathrm{Cl}_{2}(235 \mathrm{~mL}), \mathrm{CBr}_{4}(23.4 \mathrm{~g}, 70.6 \mathrm{mmol})$ and $\mathrm{n}-\mathrm{BuLi}(46 \mathrm{~mL}$ of $2.48 \mathrm{M}$ hexane solution, $113 \mathrm{mmol}$ ) were used were used. Purification of the crude product by flash column chromatography afforded $62(9.6 \mathrm{~g}, 59 \%)$ as a colorless oil: $[\alpha]^{25} \mathrm{D}=-8.5^{\circ}(\mathrm{c}$ $\left.1.8, \mathrm{CHCl}_{3}\right) ;{ }^{1} \mathrm{H}$ NMR $\left(400 \mathrm{MHz}, \mathrm{CDCl}_{3}\right) \delta 7.25(\mathrm{~d}, J=8.4 \mathrm{~Hz}, 2 \mathrm{H}), 6.87(\mathrm{~d}, J=8.4 \mathrm{~Hz}, 2 \mathrm{H})$, $4.59(\mathrm{AB}, J=10.8 \mathrm{~Hz}, 1 \mathrm{H}), 4.55(\mathrm{AB}, J=10.8 \mathrm{~Hz}, 1 \mathrm{H}), 4.30-4.11(\mathrm{~m}, 2 \mathrm{H}), 3.81(\mathrm{~s}, 3 \mathrm{H})$, $3.75-3.82(\mathrm{~m}, 1 \mathrm{H}), 3.70$ (bdd, $J=3.2,6.4 \mathrm{~Hz}, 1 \mathrm{H}), 2.32$ (ddd, $J=2.4,8.0,16.4 \mathrm{~Hz}, 1 \mathrm{H}$ ), 2.20 $(\mathrm{ddd}, J=2.4,6.8,16.4 \mathrm{~Hz}, 1 \mathrm{H}), 2.08(\mathrm{dq}, J=4.0,7.2 \mathrm{~Hz}, 1 \mathrm{H}), 2.02(\mathrm{t}, J=2.4 \mathrm{~Hz}, 1 \mathrm{H}), 1.58-1.70$ $(\mathrm{m}, 4 \mathrm{H}), 1.02(\mathrm{~d}, J=7.2 \mathrm{~Hz}, 3 \mathrm{H}), 0.90(\mathrm{app} \mathrm{dt}, J=7.6,9.6 \mathrm{~Hz}, 6 \mathrm{H}) ;{ }^{13} \mathrm{C} \mathrm{NMR}(100 \mathrm{MHz}$, $\left.\mathrm{CDCl}_{3}\right) \delta 159.2,130.6,129.2,113.7,112.5,83.2,81.0,76.8,74.1,69.6,67.6,55.1,35.2,29.0$, 23.1, 14.2, 8.1; IR (neat) 3294, 2972, 1613, 1515, $1249 \mathrm{~cm}^{-1}$; HRMS (ES+) $\mathrm{m} / \mathrm{z}$ for $\mathrm{C}_{21} \mathrm{H}_{30} \mathrm{O}_{4} \mathrm{Na}[\mathrm{M}+\mathrm{Na}]^{+}$calcd 369.2042, found 369.2033.

\section{(2R,3S)-2-(4-Methoxy-benzyloxy)-3-methyl-hex-5-ynal (63)}

The deprotection of $\mathbf{6 2}$ was accomplished using a procedure analogous to that outlined for the conversion of 18 to 19: alkyne $\mathbf{6 2}(22.5 \mathrm{~g}, 64.9 \mathrm{mmol})$ and a 4:1 mixture of $\mathrm{AcOH}$ and water $(215 \mathrm{~mL})$. The crude product was purified by flash column chromatography to afford the diol 
product $(16.9 \mathrm{~g}, 93 \%)$ as a colorless oil: $[\alpha]^{25} \mathrm{D}=+0.08^{\circ}\left(c 1.3, \mathrm{CHCl}_{3}\right) ;{ }^{1} \mathrm{H} \mathrm{NMR}(400 \mathrm{MHz}$, $\left.\mathrm{CDCl}_{3}\right) \delta 7.28(\mathrm{~d}, J=8.4 \mathrm{~Hz}, 2 \mathrm{H}), 6.89(\mathrm{~d}, J=8.4 \mathrm{~Hz}, 2 \mathrm{H}), 4.61(\mathrm{AB}, J=10.8 \mathrm{~Hz}, 1 \mathrm{H}), 4.55$ $(\mathrm{AB}, J=10.8 \mathrm{~Hz}, 1 \mathrm{H}), 3.81(\mathrm{~s}, 3 \mathrm{H}), 3.70-3.82(\mathrm{~m}, 3 \mathrm{H}), 3.65(\mathrm{dd}, J=4.0,6.0 \mathrm{~Hz}, 1 \mathrm{H}), 2.19-2.35$ $(\mathrm{m}, 3 \mathrm{H}), 2.04-2.11(\mathrm{~m}, 1 \mathrm{H}), 2.03(\mathrm{t}, J=2.4 \mathrm{~Hz}, 1 \mathrm{H}), 1.94(\mathrm{~b} \mathrm{t}, J=6.0 \mathrm{~Hz}, 1 \mathrm{H}), 1.08(\mathrm{~d}, J=6.8$ $\mathrm{Hz}, 3 \mathrm{H}) ;{ }^{13} \mathrm{C} \mathrm{NMR}\left(100 \mathrm{MHz}, \mathrm{CDCl}_{3}\right) \delta 159.2,130.3,129.4,113.8,83.1,81.1,74.3,71.8$, 69.9, 63.8, 55.2, 34.3, 23.3, 14.4; IR (neat) 3401, 3294, 2935, 1614, 1515, 1250, 1074, 1035 $\mathrm{cm}^{-1}$; HRMS (ES+) $\mathrm{m} / z$ for $\mathrm{C}_{16} \mathrm{H}_{22} \mathrm{O}_{4} \mathrm{Na}[\mathrm{M}+\mathrm{Na}]^{+}$calcd 301.1416, found 301.1418.

Oxidative cleavage of the diol above was accomplished using a procedure analogous to that outlined for the conversion of $\mathbf{1 8}$ to 19: the diol product from the previous step (16.9 g, 60.7 $\mathrm{mmol})$, THF $(100 \mathrm{~mL}), \mathrm{pH} 7$ buffer $(100 \mathrm{~mL})$ and $\mathrm{NaIO}_{4}(15.6 \mathrm{~g}, 72.9 \mathrm{mmol})$ were used. The product was used without further purification. $63(12.1 \mathrm{~g}, 81 \%)$ was a colorless oil: $[\alpha]^{25}{ }_{\mathrm{D}}=$ $+69^{\circ}\left(c 1.1, \mathrm{CHCl}_{3}\right) ;{ }^{1} \mathrm{H}$ NMR $\left(400 \mathrm{MHz}, \mathrm{CDCl}_{3}\right) \delta 9.70(\mathrm{~d}, J=1.6 \mathrm{~Hz}, 1 \mathrm{H}), 7.29(\mathrm{~d}, J=8.4$ $\mathrm{Hz}, 2 \mathrm{H}), 6.89(\mathrm{~d}, J=8.4 \mathrm{~Hz}, 2 \mathrm{H}), 4.66(\mathrm{~d}, J=11.2 \mathrm{~Hz}, 1 \mathrm{H}), 4.50(\mathrm{~d}, J=11.2 \mathrm{~Hz}, 1 \mathrm{H}), 3.94$ (bdd, $J=1.2,3.2 \mathrm{~Hz}, 1 \mathrm{H}), 3.81(\mathrm{~s}, 3 \mathrm{H}), 2.18-2.38(\mathrm{~m}, 3 \mathrm{H}), 2.00(\mathrm{t}, J=2.4 \mathrm{~Hz}, 1 \mathrm{H}), 1.00(\mathrm{~d}$, $J=6.4 \mathrm{~Hz}, 3 \mathrm{H}) ;{ }^{13} \mathrm{C}$ NMR $\left(100 \mathrm{MHz}, \mathrm{CDCl}_{3}\right) \delta 204.2,159.3,129.6,129.3,113.7,84.5,82.1$, 72.7, 70.2, 55.0, 34.7, 22.2, 14.0; IR (neat) 3291, 2936, 1731, 1514, $1250 \mathrm{~cm}^{-1}$; HRMS (ES +) $\mathrm{m} / \mathrm{z}$ for $\mathrm{C}_{15} \mathrm{H}_{18} \mathrm{O}_{3} \mathrm{Na}[\mathrm{M}+\mathrm{Na}]^{+}$calcd 269.1154, found 269.1146 .

\section{(3R,4S,5R,6S)-3-(Dimethylphenylsilanyl)-5-(4-methoxy-benzyloxy)-6-methyl-non-1-en-8- yn-4-ol (64)}

Hydroxyallylsilane $\mathbf{6 4}$ was synthesized using a procedure analogous to that outlined for the conversion of 19 to 21 : aldehyde $\mathbf{6 3}(11.5 \mathrm{~g}, 46.7 \mathrm{mmol}), 4 \AA$ mol. sieves $(8.6 \mathrm{~g})$, toluene (80 $\mathrm{mL})$ and $(S, S)-\mathbf{2 0}(110 \mathrm{~mL}$ of a $1.0 \mathrm{M}$ solution in toluene, $110 \mathrm{mmol})$ were used. The crude product was purified by flash column chromatography to afford $64(17.7 \mathrm{~g}, 90 \%)$ as a colorless oil: $[\alpha]^{25}{ }_{\mathrm{D}}=-12^{\circ}\left(c 0.8, \mathrm{CHCl}_{3}\right) ;{ }^{1} \mathrm{H}$ NMR $\left(400 \mathrm{MHz}, \mathrm{CDCl}_{3}\right) \delta$ 7.52-7.56 (m, 2H), 7.30-7.38 $(\mathrm{m}, 3 \mathrm{H}), 7.24(\mathrm{~d}, J=8.4 \mathrm{~Hz}, 2 \mathrm{H}), 6.87(\mathrm{~d}, J=8.4 \mathrm{~Hz}, 2 \mathrm{H}), 5.98(\mathrm{dt}, J=10.4,17.2 \mathrm{~Hz}, 1 \mathrm{H})$, $5.02(\mathrm{dd}, J=2.0,10.4 \mathrm{~Hz}, 1 \mathrm{H}), 4.85(\mathrm{dd}, J=2.0,17.2 \mathrm{~Hz}, 1 \mathrm{H}), 4.59(\mathrm{AB}, J=10.4 \mathrm{~Hz}, 1 \mathrm{H})$, $4.52(\mathrm{AB}, J=10.4 \mathrm{~Hz}, 1 \mathrm{H}), 3.80$ (s, $3 \mathrm{H}), 3.65$ (bdt, $J=2.4,8.4 \mathrm{~Hz}, 1 \mathrm{H}), 3.52$ (bdd, $J=2.8$, $8.4 \mathrm{~Hz}, 1 \mathrm{H}), 2.28-2.31(\mathrm{~m}, 1 \mathrm{H}), 2.20(\mathrm{ddq}, J=2.4,7.2,16.8 \mathrm{~Hz}, 2 \mathrm{H}), 1.98(\mathrm{t}, J=2.4 \mathrm{~Hz}, 1 \mathrm{H})$, $1.85-1.92(\mathrm{~m}, 1 \mathrm{H}), 1.82(\mathrm{bd}, J=10.8 \mathrm{~Hz}, 1 \mathrm{H}), 0.82(\mathrm{~d}, J=7.2 \mathrm{~Hz}, 3 \mathrm{H}), 0.38(\mathrm{~s}, 3 \mathrm{H}), 0.31(\mathrm{~s}$, $3 \mathrm{H}) ;{ }^{13} \mathrm{C}$ NMR $\left(100 \mathrm{MHz}, \mathrm{CDCl}_{3}\right) \delta 159.2,137.8,134.1,134.0,130.6,129.3,128.9,127.6$, $114.8,113.9,83.8,83.3,75.1,71.6,69.5,55.2,38.3,34.1,23.8,13.4,-3.8,-4.3$; IR (neat) 3564 , $3303,2963,1613,1514,1248 \mathrm{~cm}^{-1}$; HRMS (ES+) $\mathrm{m} / z$ for $\mathrm{C}_{26} \mathrm{H}_{34} \mathrm{O}_{3} \mathrm{SiNa}[\mathrm{M}+\mathrm{Na}]^{+}$calcd 445.2175, found 445.2173.

\section{1-[(1R,2S,3R)-3-(Dimethyl-phenyl-silanyl)-1-((S)-1-methyl-but-3-ynyl)-2-triethylsilanyloxy- pent-4-enyloxymethyl]-4-methoxy-benzene (8)}

Allylsilane 8 was protected as the triethylsilyl ether using a procedure analogous to that outlined for the conversion of 21 to $7: 64$ ( $0.53 \mathrm{~g}, 1.3 \mathrm{mmol}), \mathrm{DMF}(45 \mathrm{~mL})$, imidazole $(0.26 \mathrm{~g}, 3.8$ $\mathrm{mmol})$ and triethylsilyl chloride $(0.64 \mathrm{~mL}, 3.8 \mathrm{mmol})$ were used. The crude product was purified by flash column chromatography to afford $\mathbf{8}(0.60 \mathrm{~g}, 89 \%)$ as a colorless oil: $[\alpha]^{25}{ }_{\mathrm{D}}$ $=+9.0^{\circ}\left(c 1.4, \mathrm{CHCl}_{3}\right) ;{ }^{1} \mathrm{H} \mathrm{NMR}\left(400 \mathrm{MHz}, \mathrm{CDCl}_{3}\right) \delta$ 7.48-7.54 (m, 2H), 7.29-7.38 (m, 3H), $7.18(\mathrm{~d}, J=8.8 \mathrm{~Hz}, 2 \mathrm{H}), 6.85(\mathrm{~d}, J=8.8 \mathrm{~Hz}, 2 \mathrm{H}), 6.05(\mathrm{dt}, J=10.4,17.2 \mathrm{~Hz}, 1 \mathrm{H}), 4.98(\mathrm{dd}$, $J=2.0,10.0 \mathrm{~Hz}, 1 \mathrm{H}), 4.82(\mathrm{dd}, J=2.0,17.6 \mathrm{~Hz}, 1 \mathrm{H}), 4.45(\mathrm{~d}, J=11.6 \mathrm{~Hz}, 1 \mathrm{H}), 4.28(\mathrm{~d}, J=$ $11.2 \mathrm{~Hz}, 1 \mathrm{H}), 4.02(\mathrm{dd}, J=2.0,6.0 \mathrm{~Hz}, 1 \mathrm{H}), 3.81(\mathrm{~s}, 3 \mathrm{H}), 3.20(\mathrm{t}, J=5.2 \mathrm{~Hz}, 1 \mathrm{H}), 2.28(\mathrm{dq}$, $J=2.4,8.8 \mathrm{~Hz}, 1 \mathrm{H}), 2.18(\mathrm{dd}, J=1.6,10.4 \mathrm{~Hz}, 1 \mathrm{H}), 1.92-2.10(\mathrm{~m}, 2 \mathrm{H}), 1.91(\mathrm{t}, J=2.4 \mathrm{~Hz}$, $1 \mathrm{H}), 0.96(\mathrm{~d}, J=6.0 \mathrm{~Hz}, 3 \mathrm{H}), 0.88(\mathrm{t}, J=8.0 \mathrm{~Hz}, 9 \mathrm{H}), 0.46-0.56(\mathrm{~m}, 6 \mathrm{H}), 0.35(\mathrm{~s}, 3 \mathrm{H}), 0.30$ $(\mathrm{s}, 3 \mathrm{H}) ;{ }^{13} \mathrm{C}$ NMR $\left(100 \mathrm{MHz}, \mathrm{CDCl}_{3}\right) \delta 158.9,138.0,135.7,134.2,131.1,128.9,128.8,127.6$, 114.3, 113.5, 84.5, 83.8, 73.1, 72.6, 69.1, 55.2, 38.2, 33.8, 23.8, 15.3, 7.1, 5.5, -3.1, -4.1; IR (neat) 3310, 2956, 1614, 1514, 1248, $1112 \mathrm{~cm}^{-1}$; HRMS (ES+) $m / z$ for $\mathrm{C}_{32} \mathrm{H}_{48} \mathrm{O}_{3} \mathrm{Si}_{2} \mathrm{Na}$ [M $+\mathrm{Na}]^{+}$calcd 559.3040, found 559.3030. 
$(4 R, 5 R)-4-((E)-4-\{(2 S, 4 S, 5 R)-4-(D i m e t h y l-p h e n y l-s i l a n y l)-5-[(1 S, 2 R, 3 S)-2-(4-m e t h o x y-$ benzyloxy)-3-methyl-1-triethylsilanyloxy-hex-5-ynyl]-tetrahydro-furan-2-yl\}-but-1-enyl)-2,2dimethyl-5-vinyl-[1,3]dioxolane (65)

Tetrahydrofuran 65 was synthesized using a procedure analogous to that outlined for $\mathbf{2 4}$ : aldehyde $5(0.079 \mathrm{~g}, 0.37 \mathrm{mmol})$, allylsilane $8(0.60 \mathrm{~g}, 1.12 \mathrm{mmol})$, activated $4 \AA$ molecular sieves $(0.15 \mathrm{~g})$, dichloromethane $(0.75 \mathrm{~mL})$ and $\mathrm{BF}_{3} \cdot \mathrm{OEt}_{2}(47 \mu \mathrm{L}, 0.37 \mathrm{mmol})$ were used. Purification of the crude product by flash column chromatography afforded $65(0.171 \mathrm{~g}, 61 \%$; $0.43 \mathrm{~g}$ of allylsilane 8 was recovered $)$ as a colorless oil: $[\alpha]^{25} \mathrm{D}=+19^{\circ}\left(c 1.0, \mathrm{CHCl}_{3}\right) ;{ }^{1} \mathrm{H}$ NMR $\left(400 \mathrm{MHz}, \mathrm{CDCl}_{3}\right) \delta$ 7.45-7.50 (m, 2H), 7.36-7.35 (m, 3H), $7.24(\mathrm{~d}, J=8.8 \mathrm{~Hz}, 2 \mathrm{H})$, $6.86(\mathrm{~d}, J=8.8 \mathrm{~Hz}, 2 \mathrm{H}), 5.72-5.84(\mathrm{~m}, 2 \mathrm{H}), 5.42(\mathrm{bdd}, J=6.8,15.2 \mathrm{~Hz}, 1 \mathrm{H}), 5.32(\mathrm{~d}, J=17.2$ $\mathrm{Hz}, 1 \mathrm{H}), 5.21(\mathrm{~d}, J=10.4 \mathrm{~Hz}, 1 \mathrm{H}), 4.62(\mathrm{~d}, J=11.6 \mathrm{~Hz}, 1 \mathrm{H}), 4.39(\mathrm{~d}, J=11.6 \mathrm{~Hz}, 1 \mathrm{H}), 4.00-4.08$ $(\mathrm{m}, 2 \mathrm{H}), 3.96(\mathrm{~d}, J=8.4 \mathrm{~Hz}, 1 \mathrm{H}), 3.78(\mathrm{~s}, 3 \mathrm{H}), 3.66-3.75(\mathrm{~m}, 1 \mathrm{H}), 3.64(\mathrm{~d}, J=6.8 \mathrm{~Hz}, 1 \mathrm{H})$, 3.46 (bdd, $J=4.4,6.4 \mathrm{~Hz}, 1 \mathrm{H}), 2.32-2.42(\mathrm{~m}, 1 \mathrm{H}), 2.21-2.25(\mathrm{~m}, 4 \mathrm{H}), 1.94(\mathrm{~s}, 1 \mathrm{H}), 1.79-1.89$ $(\mathrm{m}, 1 \mathrm{H}), 1.56-1.74(\mathrm{~m}, 3 \mathrm{H}), 1.36-1.48(\mathrm{~m}, 1 \mathrm{H}), 1.43(\mathrm{~s}, 3 \mathrm{H}), 1.42(\mathrm{~s}, 3 \mathrm{H}), 0.90-1.00(\mathrm{~m}, 12 \mathrm{H})$, 0.54-0.64 (m, 6H), $0.35(\mathrm{~s}, 3 \mathrm{H}), 0.33(\mathrm{~s}, 3 \mathrm{H}) ;{ }^{13} \mathrm{C} \mathrm{NMR}\left(100 \mathrm{MHz}, \mathrm{CDCl}_{3}\right) \delta$ 158.7, 137.7, $136.4,134.3,133.9,131.3,129.1,128.6,127.8,125.6,118.3,113.5,108.7,84.2,82.7,82.2$, 82.1, 81.2, 78.0, 74.0, 73.7, 69.1, 55.1, 35.2, 34.7, 34.5, 29.2, 27.0, 26.9, 25.4, 23.5, 14.9, 7.1, 5.3, -3.7, -4.4; IR (neat) 3308, 2955, 1614, 1514, $1248 \mathrm{~cm}^{-1}$; HRMS (ES+) $\mathrm{m} / z$ for $\mathrm{C}_{44} \mathrm{H}_{66} \mathrm{O}_{6} \mathrm{Si}_{2} \mathrm{Na}[\mathrm{M}+\mathrm{Na}]^{+}$calcd 769.4296 , found 769.4307 .

\section{$(1 R, 2 R, 3 S)-1-\{(2 S, 5 S)-5-[(E)-4-((4 R, 5 R)-2,2-D i m e t h y l-5-v i n y l-[1,3] d i o x o l a n-4-y l)-b u t-3-e n y l]-$ tetrahydro-furan-2-yl\}-2-(4-methoxy-benzyloxy)-3-methyl-hex-5-yn-1-ol (66)}

The protiodesilylation of 65 was accomplished using a procedure analogous to that outlined for the conversion of $\mathbf{2 4}$ to $\mathbf{2 5}$ : [3+2] adduct $\mathbf{6 5}(1.26 \mathrm{~g}, 1.69 \mathrm{mmol})$, DMF (1.7 mL), $\mathrm{TBAF} \cdot 3 \mathrm{H}_{2} \mathrm{O}(4.66 \mathrm{~g}, 14.8 \mathrm{mmol}$, added portionwise) were used. The crude product was purified by flash column chromatography to afford $\mathbf{6 6}(0.68 \mathrm{~g}, 81 \%)$ as a colorless oil: $[\alpha]$ $25_{\mathrm{D}}=-4.1^{\circ}\left(\mathrm{c} 0.09, \mathrm{CHCl}_{3}\right) ;{ }^{1} \mathrm{H} \mathrm{NMR}\left(400 \mathrm{MHz}, \mathrm{CDCl}_{3}\right) \delta 7.27(\mathrm{~d}, J=8.4 \mathrm{~Hz}, 2 \mathrm{H}), 6.87(\mathrm{~d}$, $J=8.4 \mathrm{~Hz}, 2 \mathrm{H}), 5.73-5.83(\mathrm{~m}, 2 \mathrm{H}), 5.42(\mathrm{bdd}, J=6.0,15.2 \mathrm{~Hz}, 1 \mathrm{H}), 5.33(\mathrm{~d}, J=16.8 \mathrm{~Hz}, 1 \mathrm{H})$, 5.22 (d, $J=10.4 \mathrm{~Hz}, 1 \mathrm{H}$ ), 4.60 (app q, $J=10.8 \mathrm{~Hz}, 2 \mathrm{H}$ ), 4.01-4.07 (m, 2H), 3.90 (bdt, $J=4.8$, $5.2 \mathrm{~Hz}, 1 \mathrm{H}), 3.84(\mathrm{bt}, J=6.8 \mathrm{~Hz}, 1 \mathrm{H}), 3.79(\mathrm{~s}, 3 \mathrm{H}), 3.46-3.57(\mathrm{~m}, 2 \mathrm{H}), 2.52-2.57(\mathrm{~m}, 1 \mathrm{H}), 2.30$ (ddq, $J=2.8,7.2,16.8 \mathrm{~Hz}, 2 \mathrm{H}), 2.08-2.20(\mathrm{~m}, 3 \mathrm{H}), 1.99(\mathrm{t}, J=2.8 \mathrm{~Hz}, 1 \mathrm{H}), 1.88-1.96(\mathrm{~m}, 1 \mathrm{H})$, $1.64-1.86(\mathrm{~m}, 3 \mathrm{H}), 1.46-1.62(\mathrm{~m}, 2 \mathrm{H}), 1.43(\mathrm{~s}, 3 \mathrm{H}), 1.42(\mathrm{~s}, 3 \mathrm{H}), 1.06(\mathrm{~d}, J=6.8 \mathrm{~Hz}$, $3 \mathrm{H}) ;{ }^{13} \mathrm{C}$ NMR $\left(100 \mathrm{MHz}, \mathrm{CDCl}_{3}\right) \delta 159.2,136.0,134.3,130.6,129.5,126.0,118.5,113.8$, 108.8, 83.2, 82.2, 82.2, 81.0, 80.2, 79.3, 73.7, 73.7, 69.5, 55.2, 35.1, 35.0, 31.1, 29.1, 27.7, 27.1, 27.0, 22.8, 15.0; IR (neat) 3523, 2985, 1613, 1515, 1248, $1053 \mathrm{~cm}^{-1}$; HRMS (ES+) $\mathrm{m} /$ $z$ for $\mathrm{C}_{30} \mathrm{H}_{42} \mathrm{O}_{6} \mathrm{Na}[\mathrm{M}+\mathrm{Na}]^{+}$calcd 521.2879, found 521.2879.

\section{$(1 S, 2 R, 3 S)-1-\{(2 S, 5 S)-5-[(E)-4-((4 R, 5 R)-2,2-D i m e t h y l-5-v i n y l-[1,3] d i o x o l a n-4-y l)-b u t-3-e n y l]-$ tetrahydro-furan-2-yl\}-3-methyl-1-triethylsilanyloxy-hex-5-yn-2-ol (67)}

Protection of 66 as the triethylsilyl ether was accomplished using a procedure analogous to that outlined for the conversion of 24 to 25 : alcohol 66 ( $0.40 \mathrm{~g}, 0.80 \mathrm{mmol})$, triethylamine $(0.22$ $\mathrm{mL}, 1.6 \mathrm{mmol})$, dichloromethane $(3 \mathrm{~mL})$ and triethylsilyl trifluoromethanesulfonate $(0.22 \mathrm{~mL}$, $0.96 \mathrm{mmol}$ ) were used.

Deprotection of the $p$-methoxybenzyl ether from the above intermediate was accomplished using a procedure analogous to that outlined for the conversion of 24 to 25: dichloromethane $(10 \mathrm{~mL}), \mathrm{pH} 7 \mathrm{buffer}(1 \mathrm{~mL})$ and DDQ $(0.37 \mathrm{~g}, 1.6 \mathrm{mmol})$ were used. Purification of the crude product by flash column chromatography afforded $67(0.34 \mathrm{~g}, 85 \%)$ as a colorless oil: $[\alpha]$ $25_{\mathrm{D}}=+18^{\circ}\left(c 0.18, \mathrm{CHCl}_{3}\right) ;{ }^{1} \mathrm{H} \mathrm{NMR}\left(400 \mathrm{MHz}, \mathrm{CDCl}_{3}\right) \delta 5.74-5.85(\mathrm{~m}, 2 \mathrm{H}), 5.44$ (bddd, $J=1.2,6.0,15.6 \mathrm{~Hz}, 1 \mathrm{H}), 5.34(\mathrm{~d}, J=17.6 \mathrm{~Hz}, 1 \mathrm{H}), 5.24(\mathrm{~d}, J=10.4 \mathrm{~Hz}, 1 \mathrm{H}), 4.02-4.10(\mathrm{~m}$, $2 \mathrm{H}), 3.81-3.88(\mathrm{~m}, 1 \mathrm{H}), 3.74-3.81(\mathrm{~m}, 1 \mathrm{H}), 3.67(\mathrm{dd}, J=2.0,6.8 \mathrm{~Hz}, 1 \mathrm{H}), 3.42$ (ddd, $J=2.0$, $7.2,9.2 \mathrm{~Hz}, 1 \mathrm{H}), 2.46(\mathrm{~d}, J=8.8 \mathrm{~Hz}, 1 \mathrm{H}), 2.07-2.32(\mathrm{~m}, 4 \mathrm{H}), 1.96(\mathrm{t}, J=2.4 \mathrm{~Hz}, 1 \mathrm{H}), 1.76-1.98$ 
(m, 3H), 1.50-1.71 (m, 3H), 1.42-1.50 (m, 1H), $1.44(\mathrm{~s}, 3 \mathrm{H}), 1.44(\mathrm{~s}, 3 \mathrm{H}), 1.08(\mathrm{~d}, J=6.8 \mathrm{~Hz}$, $3 \mathrm{H}), 0.96(\mathrm{t}, J=7.6 \mathrm{~Hz}, 9 \mathrm{H}), 0.59-0.75(\mathrm{~m}, 6 \mathrm{H}) ;{ }^{13} \mathrm{C} \mathrm{NMR}\left(100 \mathrm{MHz}, \mathrm{CDCl}_{3}\right) \delta 135.9,134.3$, 125.9, 118.3, 108.7, 82.2, 82.2, 82.1, 81.1, 78.6, 75.1, 73.3, 69.8, 35.5, 35.1, 30.8, 29.1, 27.2, 27.0, 26.9, 22.7, 15.0, 6.9, 5.3; IR (neat) 3535, 3311, 2955, 1239, 1056, $741 \mathrm{~cm}^{-1}$; HRMS (ES +) $\mathrm{m} / \mathrm{z}$ for $\mathrm{C}_{28} \mathrm{H}_{48} \mathrm{O}_{5} \mathrm{SiNa}[\mathrm{M}+\mathrm{Na}]^{+}$calcd 515.3169, found 515.3160.

\section{2-epi-19-epi-Amphidinolide E (4) series}

Tricarbonyl[(E)-(2R,3S)-2-Methyl-hexa-3,5-dienoic acid $(1 R, 2 S)-1-((R)-\{(2 S, 5 S)-5-[(E)-4-((4 R$, $5 R$ )-2,2-dimethyl-5-vinyl-[1,3]dioxolan-4-yl)-but-3-enyl]-tetrahydro-furan-2-yl\}triethylsilanyloxy-methyl)-2-methyl-pent-4-ynyl ester]iron (70)

The esterification of alcohol 67 was accomplished using a procedure analogous to that outlined for 39: alcohol $67(0.33 \mathrm{~g}, 0.67 \mathrm{mmol})$, acid $10(0.22 \mathrm{~g}, 0.80 \mathrm{mmol})$, triethylamine $(0.22 \mathrm{~mL}$, $1.6 \mathrm{mmol})$, DMAP (0.082 $\mathrm{g}, 0.67 \mathrm{mmol})$, THF (1.4 mL) and 2,4,6-trichlorobenzoyl chloride $(125 \mu \mathrm{L}, 0.80 \mathrm{mmol})$ were used. Purification of the crude product by flash column chromatography afforded $70(0.418 \mathrm{~g}, 85 \%)$ as a colorless oil: $[\alpha]^{25} \mathrm{D}=-2.6^{\circ}(c 0.54$, $\left.\mathrm{CHCl}_{3}\right) ;{ }^{1} \mathrm{H} \mathrm{NMR}\left(400 \mathrm{MHz}, \mathrm{CDCl}_{3}\right) \delta 5.75-5.83(\mathrm{~m}, 2 \mathrm{H}), 5.41-5.47(\mathrm{~m}, 1 \mathrm{H}), 5.35-5.40(\mathrm{~m}$, $1 \mathrm{H}), 5.34(\operatorname{app~d}, J=23.6 \mathrm{~Hz}, 1 \mathrm{H}), 5.25-5.30(\mathrm{~m}, 1 \mathrm{H}), 5.23(\operatorname{app~d}, J=12.0 \mathrm{~Hz}, 1 \mathrm{H}), 4.89(\mathrm{dd}$, $J=2.4,7.6 \mathrm{~Hz}, 1 \mathrm{H}), 4.03-4.08(\mathrm{~m}, 2 \mathrm{H}), 3.70-3.79(\mathrm{~m}, 1 \mathrm{H}), 3.71(\mathrm{dd}, J=3.2,7.2 \mathrm{~Hz}, 1 \mathrm{H})$, $3.59-3.65(\mathrm{~m}, 1 \mathrm{H}), 2.05-2.37(\mathrm{~m}, 6 \mathrm{H}), 1.98(\mathrm{t}, J=2.0 \mathrm{~Hz}, 1 \mathrm{H}), 1.85-1.95(\mathrm{~m}, 1 \mathrm{H}), 1.75-1.85$ $(\mathrm{m}, 2 \mathrm{H}), 1.60-1.70(\mathrm{~m}, 1 \mathrm{H}), 1.50-1.60(\mathrm{~m}, 2 \mathrm{H}), 1.40-1.50(\mathrm{~m}, 1 \mathrm{H}), 1.44(\mathrm{~s}, 3 \mathrm{H}), 1.44(\mathrm{~s}, 3 \mathrm{H})$, $1.33(\mathrm{~d}, J=7.2 \mathrm{~Hz}, 3 \mathrm{H}), 1.00(\mathrm{~d}, J=6.4 \mathrm{~Hz}, 3 \mathrm{H}), 0.93-0.99(\mathrm{~m}, 1 \mathrm{H}), 0.96(\mathrm{t}, J=8.0 \mathrm{~Hz}, 9 \mathrm{H})$, 0.59-0.73 (m, 6H), 0.35 (bdd, $J=2.0,8.4 \mathrm{~Hz}, 1 \mathrm{H}) ;{ }^{13} \mathrm{C} \mathrm{NMR}\left(100 \mathrm{MHz}, \mathrm{CDCl}_{3}\right) \delta 211.0$, 173.6, 136.1, 134.3, 125.9, 118.4, 108.8, 87.1, 82.2, 82.2, 81.8, 80.4, 78.6, 77.2, 76.9, 74.9, 70.1, 64.0, 44.6, 40.4, 35.1, 33.0, 30.9, 29.2, 27.5, 27.0, 27.0, 22.7, 19.3, 15.6, 7.0, 5.3; IR (neat) 3312, 2877, 2050, 1982, 1732, 1380, $1239 \mathrm{~cm}^{-1}$; HRMS (ES+) $\mathrm{m} / \mathrm{z}$ for $\mathrm{C}_{38} \mathrm{H}_{56} \mathrm{FeO}_{9} \mathrm{SiNa}[\mathrm{M}+\mathrm{Na}]^{+}$calcd 763.2941 , found 763.2958 .

(4E,11E,13E)-(1S,6R,10R,15S,18R,19R,20S)-18-((S)-3-lodo-1-methyl-but-3-enyl)-8,8,15trimethyl-19-triethylsilanyloxy-7,9,17,23-tetraoxa-tricyclo[18.2.1.06,10]tricosa-4,11,13trien-16-one (71)

The oxidative decomplexation of 70 was accomplished using a procedure analogous to that outlined for the conversion of 39 to 40 : ester $70(173 \mathrm{mg}, 0.234 \mathrm{mmol})$, acetone $(3 \mathrm{~mL})$ and cerium ammonium nitrate (CAN) $(0.28 \mathrm{~g}, 0.51 \mathrm{mmol})$ were used. The crude product was purified by flash column chromatography to afford the polyene product $(135 \mathrm{mg}, 96 \%)$ as a colorless oil: $[\alpha]^{25} \mathrm{D}=+16^{\circ}\left(c 0.26, \mathrm{CHCl}_{3}\right) ;{ }^{1} \mathrm{H} \mathrm{NMR}\left(400 \mathrm{MHz}, \mathrm{CDCl}_{3}\right) \delta 6.29(\mathrm{dt}, J=10.4$, $16.8,1 \mathrm{H}), 6.14(\mathrm{dd}, J=10.4,15.2 \mathrm{~Hz}, 1 \mathrm{H}), 5.71-5.84(\mathrm{~m}, 3 \mathrm{H}), 5.39-5.47(\mathrm{~m}, 1 \mathrm{H}), 5.32(\mathrm{~d}, J$ $=17.6 \mathrm{~Hz}, 1 \mathrm{H}), 5.22(\mathrm{~d}, J=11.6 \mathrm{~Hz}, 1 \mathrm{H}), 5.16(\mathrm{~d}, J=16.4,1 \mathrm{H}), 5.05(\mathrm{~d}, J=10.0 \mathrm{~Hz}, 1 \mathrm{H})$, $4.88(\mathrm{dd}, J=2.8,7.6 \mathrm{~Hz}, 1 \mathrm{H}), 4.01-4.07(\mathrm{~m}, 2 \mathrm{H}), 3.61-3.77(\mathrm{~m}, 3 \mathrm{H}), 3.20$ (quint., $J=7.2 \mathrm{~Hz}$, $1 \mathrm{H}), 2.26-2.37(\mathrm{~m}, 1 \mathrm{H}), 2.04-2.26(\mathrm{~m}, 4 \mathrm{H}), 1.96(\mathrm{t}, J=2.0 \mathrm{~Hz}, 1 \mathrm{H}), 1.75-1.93(\mathrm{~m}, 2 \mathrm{H})$, $1.49-1.70(\mathrm{~m}, 3 \mathrm{H}), 1.40-1.46(\mathrm{~m}, 1 \mathrm{H}), 1.43(\mathrm{~s}, 3 \mathrm{H}), 1.42(\mathrm{~s}, 3 \mathrm{H}), 1.29(\mathrm{~d}, J=7.2 \mathrm{~Hz}, 3 \mathrm{H}), 0.96$ $(\mathrm{d}, J=5.6 \mathrm{~Hz}, 3 \mathrm{H}), 0.95(\mathrm{t}, J=8.0 \mathrm{~Hz}, 9 \mathrm{H}), 0.56-0.72(\mathrm{~m}, 6 \mathrm{H}) ;{ }^{13} \mathrm{C} \mathrm{NMR}(100 \mathrm{MHz}$, $\left.\mathrm{CDCl}_{3}\right) \delta 173.7,136.4,136.2,134.3,132.6,132.2,125.9,118.5,117.0,108.8,82.2,82.2,82.0$, 80.3, 78.6, 76.8, 74.9, 70.0, 43.1, 35.1, 33.1, 30.9, 29.2, 27.5, 27.0, 27.0, 22.7, 17.1, 15.4, 7.0, 5.3; IR (neat) 3311, 2954, 2877, 1733, 1456, 1240, $1171 \mathrm{~cm}^{-1}$; HRMS (ES+) $\mathrm{m} / z$ for $\mathrm{C}_{35} \mathrm{H}_{56} \mathrm{O}_{6} \mathrm{SiNa}[\mathrm{M}+\mathrm{Na}]^{+}$calcd 623.3744, found 623.3751 .

The ring closing metathesis of the polyene intermediate was accomplished by using a procedure analogous to that outlined for the conversion of $\mathbf{3 9}$ of 40: polyene from the preceding step (40 $\mathrm{mg}, 0.066 \mathrm{mmol})$, dichloromethane $(66 \mathrm{~mL})$ and Grubbs' first generation catalyst $(11 \mathrm{mg}, 0.013$ $\mathrm{mmol}$ ) were used. The crude product was purified by flash column chromatography to afford the macrocycle product $(22 \mathrm{mg}, 58 \%)$ as a colorless oil. In addition, an inseparable mixture of 
products thought to arrive by enyne metathesis $(3.8 \mathrm{mg}, 10 \%)$ was also isolated. Spectroscopic data for macrocycle product: $[\alpha]^{25} \mathrm{D}=-48^{\circ}\left(\mathrm{c} 0.10, \mathrm{CHCl}_{3}\right) ;{ }^{1} \mathrm{H} \mathrm{NMR}\left(400 \mathrm{MHz}, \mathrm{CDCl}_{3}\right) \delta$ 6.15-6.28 (m, 2H), $5.94(\mathrm{dd}, J=4.8,14.4 \mathrm{~Hz}, 1 \mathrm{H}), 5.72(\mathrm{ddd}, J=3.6,9.6,15.2 \mathrm{~Hz}, 1 \mathrm{H}), 5.57$ $(\mathrm{dd}, J=8.8,14.4 \mathrm{~Hz}, 1 \mathrm{H}), 5.34(\mathrm{dd}, J=8.4,15.2 \mathrm{~Hz}, 1 \mathrm{H}), 4.74(\mathrm{~d}, J=8.4 \mathrm{~Hz}, 1 \mathrm{H}), 3.99-4.08$ $(\mathrm{m}, 2 \mathrm{H}), 3.72(\mathrm{~d}, J=8.4 \mathrm{~Hz}, 1 \mathrm{H}), 3.29-3.38(\mathrm{~m}, 1 \mathrm{H}), 3.18-3.30(\mathrm{~m}, 2 \mathrm{H}), 2.18-2.36(\mathrm{~m}, 4 \mathrm{H})$, $1.80-2.20(\mathrm{~m}, 2 \mathrm{H}), 1.98(\mathrm{bs}, 1 \mathrm{H}), 1.64-1.74(\mathrm{~m}, 1 \mathrm{H}), 1.40-1.57(\mathrm{~m}, 2 \mathrm{H}), 1.43(\mathrm{~s}, 6 \mathrm{H}), 1.31(\mathrm{~d}$, $J=6.8 \mathrm{~Hz}, 3 \mathrm{H}), 1.15-1.32(\mathrm{~m}, 2 \mathrm{H}), 1.00(\mathrm{~d}, J=6.4 \mathrm{~Hz}, 3 \mathrm{H}), 0.95(\mathrm{t}, J=8.0 \mathrm{~Hz}, 9 \mathrm{H}), 0.53-0.70$ $(\mathrm{m}, 6 \mathrm{H}) ;{ }^{13} \mathrm{C} \mathrm{NMR}\left(100 \mathrm{MHz}, \mathrm{CDCl}_{3}\right) \delta 172.4,138.1,136.0,134.6,128.8,127.4,125.9,109.0$, 83.1, 82.2, 81.5, 79.8, 77.8, 77.4, 77.2, 75.0, 70.2, 43.3, 32.2, 32.1, 29.6, 28.5, 27.2, 27.1, 22.1, 15.8, 12.0, 7.1, 5.6; IR (neat) 3309, 2934, 2874, 1727, 1458, 1378, 1238, $1052 \mathrm{~cm}^{-1}$; HRMS (ES+) $m / z$ for $\mathrm{C}_{33} \mathrm{H}_{52} \mathrm{O}_{6} \mathrm{SiNa}[\mathrm{M}+\mathrm{Na}]^{+}$calcd 595.3431, found 595.3439.

The stannylalumination-protonolysis of the alkyne from the preceding step was accomplished using a procedure analogous to that outlined for the conversion of $\mathbf{4 0}$ to $\mathbf{4 1}$ : macrocycle alkyne $(51 \mathrm{mg}, 0.089 \mathrm{mmol}), \mathrm{THF}(1.5 \mathrm{~mL}), \mathrm{Bu}_{3} \mathrm{Sn}_{-\mathrm{AlEt}_{2}}(1.3 \mathrm{~mL}$ of the $0.42 \mathrm{M}$ solution, $0.53 \mathrm{mmol}$ ) and $\mathrm{CuCN}$ ( $2 \mathrm{mg}, 0.024 \mathrm{mmol}$ ) were used. Purification of the crude product by flash column chromatography afforded the vinylstannane product $(40 \mathrm{mg}, 52 \%)$ as a colorless oil: $[\alpha]^{25} \mathrm{D}=$ $-138^{\circ}$ ( $\left.c 0.05, \mathrm{CHCl}_{3}\right) ;{ }^{1} \mathrm{H}$ NMR $\left(400 \mathrm{MHz}, \mathrm{CDCl}_{3}\right.$ ) $\delta$ 6.15-6.29 (m, 2H), 5.95 (dd, $J=4.8$, $14.8 \mathrm{~Hz}, 1 \mathrm{H}), 5.67-5.78(\mathrm{~m}, 2 \mathrm{H}), 5.57(\mathrm{dd}, J=8.4,14.0 \mathrm{~Hz}, 1 \mathrm{H}), 5.35(\mathrm{dd}, J=8.0,15.2 \mathrm{~Hz}$, $1 \mathrm{H}), 5.20\left(\right.$ app t, $\left.{ }^{3} J_{S n-H}=34.0 \mathrm{~Hz}, 1 \mathrm{H}\right), 4.58(\mathrm{~d}, J=6.4 \mathrm{~Hz}, 1 \mathrm{H}), 4.04$ (app quint., $J=9.6 \mathrm{~Hz}$, $2 \mathrm{H}), 3.68(\mathrm{~d}, J=8.4 \mathrm{~Hz}, 1 \mathrm{H}), 3.20-3.38(\mathrm{~m}, 3 \mathrm{H}), 2.48(\mathrm{bd}, J=18.0 \mathrm{~Hz}, 1 \mathrm{H}), 2.26-2.35(\mathrm{~m}$, $1 \mathrm{H}), 2.09-2.19(\mathrm{~m}, 1 \mathrm{H}), 1.84-2.06(\mathrm{~m}, 3 \mathrm{H}), 1.64-1.74(\mathrm{~m}, 1 \mathrm{H}), 1.41-1.57(\mathrm{~m}, 8 \mathrm{H}), 1.44(\mathrm{~s}, 6 \mathrm{H})$, $1.24-1.36(\mathrm{~m}, 11 \mathrm{H}), 0.96(\mathrm{t}, J=8.0 \mathrm{~Hz}, 9 \mathrm{H}), 0.85-0.92(\mathrm{~m}, 15 \mathrm{H}), 0.82(\mathrm{~d}, J=6.8 \mathrm{~Hz}, 3 \mathrm{H})$, $0.58-0.68(\mathrm{~m}, 6 \mathrm{H}) ;{ }^{13} \mathrm{C} \mathrm{NMR}\left(100 \mathrm{MHz}, \mathrm{CDCl}_{3}\right) \delta 172.5,152.9,138.2,136.0,134.7,128.8$, 127.3, 126.7, 125.9, 109.0, 83.1, 82.2, 80.1, 78.6, 76.0, 44.2, 43.5, 33.2, 32.3, 29.7, 29.1, 28.5, 27.4, 27.2, 27.1, 15.5, 13.7, 12.0, 10.7, 10.5, 9.5, 7.1, 5.7; IR (neat) 2930, 1727, 1239, 1052 $\mathrm{cm}^{-1}$; HRMS (ES+) $\mathrm{m} / z$ for $\mathrm{C}_{45} \mathrm{H}_{80} \mathrm{O}_{6} \mathrm{SiSnNa}[\mathrm{M}+\mathrm{Na}]^{+}$calcd 887.4644, found 887.4681.

Iododestannylation of the vinylstannane intermediate was accomplished using a procedure analogous to that outlined for the conversion of $\mathbf{4 0}$ to $\mathbf{4 1}$ : vinylstannane from above (11 $\mathrm{mg}$, $0.013 \mathrm{mmol})$, dichloromethane $(1 \mathrm{~mL})$ and NIS $(3.2 \mathrm{mg}, 0.014 \mathrm{mmol})$ were used. The crude product was purified by flash column chromatography to afford $\mathbf{7 1}(9 \mathrm{mg}, 98 \%)$ as a colorless oil: $[\alpha]^{25} \mathrm{D}=-46^{\circ}\left(c 0.07, \mathrm{CHCl}_{3}\right) ;{ }^{1} \mathrm{H}$ NMR $\left(400 \mathrm{MHz}, \mathrm{CDCl}_{3}\right) \delta 6.15-6.29(\mathrm{~m}, 2 \mathrm{H}), 6.09(\mathrm{~s}$, $1 \mathrm{H}), 5.89-5.98(\mathrm{~m}, 1 \mathrm{H}), 5.76(\mathrm{~s}, 1 \mathrm{H}), 5.68-5.76(\mathrm{~m}, 1 \mathrm{H}), 5.58(\mathrm{dd}, J=8.8,12.4 \mathrm{~Hz}, 1 \mathrm{H}), 5.34$ $(\mathrm{dd}, J=8.0,14.8 \mathrm{~Hz}, 1 \mathrm{H}), 4.59(\mathrm{~d}, J=7.2 \mathrm{~Hz}, 1 \mathrm{H}), 3.98-4.90(\mathrm{~m}, 2 \mathrm{H}), 3.66(\mathrm{~d}, J=8.4 \mathrm{~Hz}$, $1 \mathrm{H}), 3.20-3.37(\mathrm{~m}, 3 \mathrm{H}), 2.61$ (bd, $J=13.6 \mathrm{~Hz}, 1 \mathrm{H}), 2.26-2.38(\mathrm{~m}, 2 \mathrm{H}), 2.08-2.17(\mathrm{~m}, 1 \mathrm{H})$, $1.84-2.02(\mathrm{~m}, 2 \mathrm{H}), 1.62-1.74(\mathrm{~m}, 1 \mathrm{H}), 1.40-1.54(\mathrm{~m}, 1 \mathrm{H}), 1.43(\mathrm{~s}, 6 \mathrm{H}), 1.34(\mathrm{~d}, J=6.8 \mathrm{~Hz}$, $3 \mathrm{H}), 1.12-1.30(\mathrm{~m}, 1 \mathrm{H}), 0.97(\mathrm{t}, J=8.0 \mathrm{~Hz}, 9 \mathrm{H}), 0.84(\mathrm{~d}, J=6.8 \mathrm{~Hz}, 3 \mathrm{H}), 0.61-0.69(\mathrm{~m}$, $6 \mathrm{H}) ;{ }^{13} \mathrm{C}$ NMR $\left(100 \mathrm{MHz}, \mathrm{CDCl}_{3}\right) \delta 172.5,138.2,136.0,134.3,128.9,127.5,127.4,126.0$, 110.6, 109.0, 83.1, 82.2, 79.9, 77.6, 77.4, 75.9, 48.5, 43.4, 33.3, 32.2, 29.7, 29.6, 28.5, 27.2, 27.1, 14.7, 12.1, 7.2, 5.6; IR (neat) 2934, 1726, 1238, $1052 \mathrm{~cm}^{-1}$; HRMS (ES+) $\mathrm{m} / z$ for $\mathrm{C}_{33} \mathrm{H}_{53} \mathrm{IO}_{6} \mathrm{SiNa}[\mathrm{M}+\mathrm{Na}]^{+}$calcd 723.2554 , found 723.2567 .

\section{2-epi-19-epi-amphidinolide E (4)}

Deprotection of $\mathbf{7 1}$ was accomplished using a procedure analogous to that outlined for the conversion of 41 to 2: vinyl iodide 71 (26 mg, $0.037 \mathrm{mmol})$ and AcOH, THF and water (4/1/1 ratio) $(1 \mathrm{~mL})$ were used. Analysis of the crude product by ${ }^{1} \mathrm{H}$ NMR indicated a 2:1 mixture of the desired C(18) and undesired C(17) lactones.

Stille coupling of the crude mixure of lactones from above was accomplished using a procedure analogous to that outlined for the conversion of $\mathbf{4 1}$ to 2 : $\mathrm{CuCl}$ (18 mg, $0.18 \mathrm{mmol}$ ), THF ( 0.5 $\mathrm{mL}$ ), vinylstannane $6(61 \mathrm{mg}, 0.17 \mathrm{mmol})$ and $\mathrm{Pd}\left(\mathrm{PPh}_{3}\right)_{4}(8 \mathrm{mg}, 0.007 \mathrm{mmol})$ were used. The crude product was purified by flash column chromatography using $10 \%$ methanol/chloroform 
to afford material that was still contaminated with an organotin impurity. The stannane impurity was removed by HPLC purification with $100 \%$ ethyl acetate eluent on a normal phase, Varian Dynamax Microsorb 60-8 Si, $250 \times 21.4 \mathrm{~mm}$ column. The retention time for 2-epi-19-epiamphidinolide $\mathrm{E}$ was $7.6 \mathrm{~min}$. The flow rate was $18 \mathrm{~mL} / \mathrm{min}$. 2-epi-19-epi-mphidinolide $\mathrm{E}$ was detected using UV absorption ( $\lambda=254 \mathrm{~nm}$ and $280 \mathrm{~nm}$ ) and RI detection. Using the above conditions $6 \mathrm{mg}$ (32\% from 71) of pure 2-epi-19-epi-amphidinolide $\mathrm{E}$ was isolated: $[\alpha]^{25} \mathrm{D}=$ $-51^{\circ}\left(c 0.10, \mathrm{CHCl}_{3}\right) ;{ }^{1} \mathrm{H}$ NMR $\left(400 \mathrm{MHz}, \mathrm{CDCl}_{3}\right) \delta 6.15-6.25(\mathrm{~m}, 2 \mathrm{H}), 6.05(\mathrm{~d}, J=16.0 \mathrm{~Hz}$, $1 \mathrm{H}), 5.94-6.01(\mathrm{~m}, 1 \mathrm{H}), 5.85(\mathrm{dt}, J=7.2,15.6 \mathrm{~Hz}, 1 \mathrm{H}), 5.55-5.70(\mathrm{~m}, 2 \mathrm{H}), 5.30(\mathrm{dd}, J=7.6$, $15.2 \mathrm{~Hz}, 1 \mathrm{H}), 4.98(\mathrm{~s}, 1 \mathrm{H}), 4.88(\mathrm{~s}, 1 \mathrm{H}), 4.74(\mathrm{~s}, 1 \mathrm{H}), 4.70(\mathrm{~s}, 1 \mathrm{H}), 4.69(\mathrm{~d}, J=10.4 \mathrm{~Hz}, 1 \mathrm{H})$, 3.90-4.01 (m, 2H), 3.71-3.77 (m, 1H), 3.46-3.54 (m, 1H), 3.38-3.46 (m, 1H), 3.29-3.38 (m, $1 \mathrm{H}), 2.78(\mathrm{bd}, J=6.4 \mathrm{~Hz}, 2 \mathrm{H}), 2.60(\mathrm{dd}, J=3.6,12.8 \mathrm{~Hz}, 1 \mathrm{H}), 2.22-2.41(\mathrm{~m}, 5 \mathrm{H}), 1.73-1.96$ $(\mathrm{m}, 4 \mathrm{H}), 1.72(\mathrm{~s}, 3 \mathrm{H}), 1.36-1.70(\mathrm{~m}, 4 \mathrm{H}), 1.34(\mathrm{~d}, J=6.8 \mathrm{~Hz}, 3 \mathrm{H}), 0.82(\mathrm{~d}, J=6.8 \mathrm{~Hz}$, $3 \mathrm{H}) ;{ }^{13} \mathrm{C}$ NMR $\left(100 \mathrm{MHz}, \mathrm{CDCl}_{3}\right) \delta 172.7,144.8,144.0,134.8,134.2,134.1,133.1,131.2$, 129.7, 129.6, 128.4, 116.2, 110.9, 79.9, 78.2, 77.3, 76.5, 73.3, 43.2, 41.5, 36.1, 32.8, 32.3, 30.2, 29.1, 27.4, 22.7, 15.7, 13.4; IR (neat) 3401, 2931, 1724, 1238, 1047, $991 \mathrm{~cm}^{-1}$; HRMS (ES+) $\mathrm{m} / \mathrm{z}$ for $\mathrm{C}_{30} \mathrm{H}_{44} \mathrm{O}_{6} \mathrm{Na}[\mathrm{M}+\mathrm{Na}]^{+}$calcd 523.3036, found 523.3030.

\section{Amphidinolide E (1) series}

\section{tert-Butyl-dimethyl-((E)-(R)-2-methyl-hexa-3,5-dienyloxy)-silane (75)}

To a $-78{ }^{\circ} \mathrm{C}$ solution of $\mathbf{4 5}^{49}$ (4.19 g, $\left.20.7 \mathrm{mmol}\right)$ and $\gamma$-(TMS)-allyltributylstannane $(11.7 \mathrm{~g}$, $29.0 \mathrm{mmol})$ in $\mathrm{CH}_{2} \mathrm{Cl}_{2}(21 \mathrm{~mL})$ was added $\mathrm{BF}_{3} \cdot \mathrm{OEt}_{2}(2.89 \mathrm{~mL}, 22.8 \mathrm{mmol})$. The reaction was stirred for $30 \mathrm{~min}$, then quenched at $-78{ }^{\circ} \mathrm{C}$ with sat. aq. $\mathrm{NaHCO}_{3}(60 \mathrm{~mL})$ and extracted with $\mathrm{CH}_{2} \mathrm{Cl}_{2}(40 \mathrm{~mL} \times 3)$. The organic phase was washed with brine, dried over anhydrous $\mathrm{MgSO}_{4}$, filtered and concentrated. The residual was dissolved in THF $(20 \mathrm{~mL})$ and treated with $\mathrm{KOt}-\mathrm{Bu}(2.0 \mathrm{~g}, 18 \mathrm{mmol})$. The reaction was stirred for $3 \mathrm{~h}$ at room temperature and then poured into a separatory funnel containing $\mathrm{Et}_{2} \mathrm{O}(100 \mathrm{~mL})$ and sat. aq. $\mathrm{NaHCO}_{3}(200 \mathrm{~mL})$. The aqueous layer was extracted with $\mathrm{Et}_{2} \mathrm{O}(50 \mathrm{~mL} \times 3)$. The organic phase was washed with brine, dried over anhydrous $\mathrm{MgSO}_{4}$, filtered and concentrated. Purification of the crude product by flash column chromatography afforded $75(2.75 \mathrm{~g}, 59 \%)$ as a colorless oil: $[\alpha]^{25} \mathrm{D}=+11^{\circ}(c$ 4.8, $\left.\mathrm{CHCl}_{3}\right) ;{ }^{1} \mathrm{H}$ NMR $\left(400 \mathrm{MHz}, \mathrm{CDCl}_{3}\right) \delta 6.31(\mathrm{dt}, J=10.4,16.8 \mathrm{~Hz}, 1 \mathrm{H}), 6.08(\mathrm{dd}, J=10.4$, $15.2 \mathrm{~Hz}, 1 \mathrm{H}), 5.64(\mathrm{dd}, J=7.2,15.2 \mathrm{~Hz}, 1 \mathrm{H}), 5.11(\mathrm{~d}, J=17.2 \mathrm{~Hz}, 1 \mathrm{H}), 4.98(\mathrm{~d}, J=10.0 \mathrm{~Hz}$, $1 \mathrm{H}), 3.51(\mathrm{dd}, J=6.0,9.6 \mathrm{~Hz}, 1 \mathrm{H}), 3.41(\mathrm{dd}, J=6.8,9.6 \mathrm{~Hz}, 1 \mathrm{H}), 2.37$ (sept., $J=6.8 \mathrm{~Hz}, 1 \mathrm{H}$ ), $1.01(\mathrm{~d}, J=6.8 \mathrm{~Hz}, 3 \mathrm{H}), 0.89(\mathrm{~s}, 9 \mathrm{H}), 0.04(\mathrm{~s}, 6 \mathrm{H}) ;{ }^{13} \mathrm{C} \mathrm{NMR}\left(100 \mathrm{MHz}, \mathrm{CDCl}_{3}\right) \delta^{3} 137.6$, 137.4, 130.5, 115.2, 67.9, 39.3, 25.9, 18.3, 16.4, -5.3, -5.4; IR (neat) 2957, 1799, 1472, 1256, $1115,1088,837 \mathrm{~cm}^{-1}$; HRMS (EI+) $\mathrm{m} / z$ for $\mathrm{C}_{9} \mathrm{H}_{17} \mathrm{OSi}\left[\mathrm{M}-\mathrm{C}_{4} \mathrm{H}_{9}\right]^{+}$calcd 169.1049 , found 169.1044 .

\section{Tricarbonyl[(E)-(2S,3R)-2-Methyl-hexa-3,5-dienoic acid (1R,2R)-1-((R)-\{(2S,5S)-5-[(E)-4-((4R, $5 R$ )-2,2-dimethyl-5-vinyl-[1,3]dioxolan-4-yl)-but-3-enyl]-tetrahydro-furan-2-yl\}- triethylsilanyloxy-methyl)-2-methyl-pent-4-ynyl ester]iron (76)}

The esterification of alcohol 25 to acid 9 was accomplished using a procedure analogous to that outlined for 39: alcohol 25 (120 mg, $0.244 \mathrm{mmol})$, acid 9 (105 mg, $0.390 \mathrm{mmol})$, triethylamine ( $0.12 \mathrm{~mL}, 0.854 \mathrm{mmol})$, DMAP (30 mg, $0.244 \mathrm{mmol})$, THF ( $0.5 \mathrm{~mL}$ ) and 2,4,6trichlorobenzoyl chloride $(61 \mu \mathrm{L}, 0.390 \mathrm{mmol})$ were used. Purification of the crude product by flash column chromatography afforded $76(179 \mathrm{mg}, 99 \%)$ as a colorless oil: $[\alpha]^{25} \mathrm{D}=+13^{\circ}$ (c $\left.0.18, \mathrm{CHCl}_{3}\right) ;{ }^{1} \mathrm{H}$ NMR $\left(400 \mathrm{MHz}, \mathrm{CDCl}_{3}\right) \delta 5.74-5.81(\mathrm{~m}, 2 \mathrm{H}), 5.43$ (app dd, $J=4.8,8.4$ $\mathrm{Hz}, 2 \mathrm{H}), 5.33(\mathrm{~d}, J=16.8 \mathrm{~Hz}, 1 \mathrm{H}), 5.21-5.26(\mathrm{~m}, 1 \mathrm{H}), 5.22(\mathrm{~d}, J=11.6 \mathrm{~Hz}, 1 \mathrm{H}), 4.69$ (dd, $J$ $=1.2,9.2 \mathrm{~Hz}, 1 \mathrm{H}), 4.04(\operatorname{app~q}, J=6.8 \mathrm{~Hz}, 2 \mathrm{H}), 3.72$ (quint., $J=5.6,1 \mathrm{H}), 3.67$ (dd, $J=1.6$, $7.6 \mathrm{~Hz}, 1 \mathrm{H}), 3.60(\operatorname{app~q}, J=8.0 \mathrm{~Hz}, 1 \mathrm{H}), 1.99-2.32(\mathrm{~m}, 7 \mathrm{H}), 1.94(\mathrm{t}, J=2.8 \mathrm{~Hz}, 1 \mathrm{H}), 1.73-1.88$ $(\mathrm{m}, 3 \mathrm{H}), 1.50-1.66(\mathrm{~m}, 2 \mathrm{H}), 1.38-1.48(\mathrm{~m}, 1 \mathrm{H}), 1.44(\mathrm{~s}, 3 \mathrm{H}), 1.43(\mathrm{~s}, 3 \mathrm{H}), 1.31(\mathrm{~d}, J=6.8 \mathrm{~Hz}$, $3 \mathrm{H}), 1.08(\mathrm{~d}, J=6.8 \mathrm{~Hz}, 3 \mathrm{H}), 0.98(\mathrm{t}, J=8.0 \mathrm{~Hz}, 9 \mathrm{H}), 0.97(\operatorname{app~d}, J=8.0 \mathrm{~Hz}, 1 \mathrm{H}), 0.59-0.76$ 
(m, 6H), 0.32 (bdd, $J=1.6,9.2 \mathrm{~Hz}, 1 \mathrm{H}) ;{ }^{13} \mathrm{C}$ NMR $\left(100 \mathrm{MHz}, \mathrm{CDCl}_{3}\right) \delta 211.2,173.7,136.1$, 134.3, 126.0, 118.5, 108.9, 87.5, 82.2, 82.2, 82.2, 80.7, 78.5, 77.4, 77.2, 75.4, 69.7, 63.9, 44.5, $40.4,35.2,33.3,30.9,29.2,27.8,27.1,26.9,22.3,19.2,16.1,7.1,5.4$; IR (neat) 3310, 2049, $1978,1732,1238,1170 \mathrm{~cm}^{-1}$; HRMS (ES+) $m / z$ for $\mathrm{C}_{38} \mathrm{H}_{56} \mathrm{FeO}_{9} \mathrm{SiNa}[\mathrm{M}+\mathrm{Na}]^{+}$calcd 763.2941, found 763.2944.

\section{$(E)-(R)-2-M e t h y l-h e x a-3,5-d i e n o i c$ acid $(1 R, 2 R)-1-((R)-\{(2 S, 5 S)-5-[(E)-4-((4 R, 5 R)-2,2-$ dimethyl-5-vinyl-[1,3]dioxolan-4-yl)-but-3-enyl]-tetrahydro-furan-2-yl\}-triethylsilanyloxy- methyl)-2-methyl-pent-4-ynyl ester (72)}

The oxidative decomplexation of 76 was accomplished using a procedure analogous to that outlined for the conversion of 39 to $40: 76(45 \mathrm{mg}, 0.061 \mathrm{mmol})$, acetone $(1 \mathrm{~mL})$ and cerium ammonium nitrate (CAN) $(67 \mathrm{mg}, 0.122 \mathrm{mmol})$ were used. The crude product was purified by flash column chromatography to afford $72(35 \mathrm{mg}, 95 \%)$ as a colorless oil: $[\alpha]^{25} \mathrm{D}=-1.0^{\circ}$ $\left(c 0.10, \mathrm{CHCl}_{3}\right) ;{ }^{1} \mathrm{H} \mathrm{NMR}\left(400 \mathrm{MHz}, \mathrm{CDCl}_{3}\right) \delta 6.29(\mathrm{dt}, J=10.0,16.8,1 \mathrm{H}), 6.15(\mathrm{dd}, J=10.4$, $15.2 \mathrm{~Hz}, 1 \mathrm{H}), 5.72-5.82(\mathrm{~m}, 3 \mathrm{H}), 5.42$ (bdd, $J=1.6,6.0,15.6 \mathrm{~Hz}, 1 \mathrm{H}), 5.32(\mathrm{~d}, J=16.4 \mathrm{~Hz}$, $1 \mathrm{H}), 5.22(\mathrm{dd}, J=1.2,10.4 \mathrm{~Hz}, 1 \mathrm{H}), 5.17(\mathrm{~d}, J=17.6,1 \mathrm{H}), 5.06(\mathrm{~d}, J=10.0 \mathrm{~Hz}, 1 \mathrm{H}), 4.70$ (dd, $J=2.0,8.8 \mathrm{~Hz}, 1 \mathrm{H}$ ), 4.04 (app q, $J=7.2 \mathrm{~Hz}, 2 \mathrm{H}$ ), 3.71 (quint., $J=5.6 \mathrm{~Hz}, 1 \mathrm{H}$ ), 3.67 (dd, $J=2.0,7.2 \mathrm{~Hz}, 1 \mathrm{H}$ ), 3.59 (q, $J=6.4 \mathrm{~Hz}, 1 \mathrm{H}), 3.21$ (quint., $J=7.2 \mathrm{~Hz}, 1 \mathrm{H}), 1.98-2.36(\mathrm{~m}, 5 \mathrm{H})$, $1.94(\mathrm{t}, J=2.4 \mathrm{~Hz}, 1 \mathrm{H}), 1.74-1.88(\mathrm{~m}, 2 \mathrm{H}), 1.57-1.66(\mathrm{~m}, 1 \mathrm{H}), 1.46-1.56(\mathrm{~m}, 1 \mathrm{H}), 1.43(\mathrm{~s}, 3 \mathrm{H})$, $1.42(\mathrm{~s}, 3 \mathrm{H}), 1.36-1.45(\mathrm{~m}, 2 \mathrm{H}), 1.29(\mathrm{~d}, J=6.8 \mathrm{~Hz}, 3 \mathrm{H}), 1.08(\mathrm{~d}, J=6.4 \mathrm{~Hz}, 3 \mathrm{H}), 0.95(\mathrm{t}, J=$ $6.8 \mathrm{~Hz}, 9 \mathrm{H}), 0.57-0.72(\mathrm{~m}, 6 \mathrm{H}) ;{ }^{13} \mathrm{C}$ NMR $\left(100 \mathrm{MHz}, \mathrm{CDCl}_{3}\right) \delta 173.7,136.3,136.2,134.3$, $132.5,132.3,125.8,118.5,117.0,108.8,82.4,82.2,82.2,80.4,78.4,77.4,75.3,69.6,42.8$, $35.2,33.3,30.9,29.2,27.0,26.9,22.2,17.0,16.1,7.0,5.3$; IR (neat) 3310, 2953, 2876, 1733 , $1378,1239 \mathrm{~cm}^{-1}$; HRMS (ES+) $\mathrm{m} / z$ for $\mathrm{C}_{35} \mathrm{H}_{56} \mathrm{O}_{6} \mathrm{SiNa}[\mathrm{M}+\mathrm{Na}]^{+}$calcd 623.3744 , found 623.3748 .

\section{(4E,11E,13E)-(1S,6R,10R,15R,18R,19R,20S)-18-((R)-3-lodo-1-methyl-but-3-enyl)-8,8,15- trimethyl-19-triethylsilanyloxy-7,9,17,23-tetraoxa-tricyclo[18.2.1.06,10]tricosa-4,11,13- trien-16-one (77)}

The ring closing metathesis of 72 was accomplished using a procedure analogous to that outlined for the conversion of 39 to 40: polyene $72(63 \mathrm{mg}, 0.105 \mathrm{mmol})$, dichloromethane $(105 \mathrm{~mL})$ and Grubbs' first generation catalyst $(17 \mathrm{mg}, 0.021 \mathrm{mmol})$ were used. The crude product was purified by flash column chromatography to afford the macrocycle product (44 $\mathrm{mg}, 73 \%$ ) as a colorless oil. In addition, an inseparable mixture of products thought to arrive by enyne metathesis $(4 \mathrm{mg}, 10 \%)$ was also isolated. Spectroscopic data for the macrocycle product: $[\alpha]^{25} \mathrm{D}=-34^{\circ}\left(c 0.21, \mathrm{CHCl}_{3}\right) ;{ }^{1} \mathrm{H} \mathrm{NMR}\left(400 \mathrm{MHz}, \mathrm{CDCl}_{3}\right) \delta 6.15-6.26(\mathrm{~m}, 2 \mathrm{H})$, 5.72 (ddd, $J=3.6,9.6,15.2 \mathrm{~Hz}, 1 \mathrm{H}), 5.49-5.57(\mathrm{~m}, 2 \mathrm{H}), 5.33$ (app dd, $J=8.4,15.6 \mathrm{~Hz}, 1 \mathrm{H})$, 4.55 (app dd, $J=1.6,9.6 \mathrm{~Hz}, 1 \mathrm{H}$ ), 4.02 (app dt, $J=8.4,26.0 \mathrm{~Hz}, 2 \mathrm{H}$ ), 3.71 (app dd, $J=1.6$, $8.4 \mathrm{~Hz}, 1 \mathrm{H}), 3.20-3.35(\mathrm{~m}, 3 \mathrm{H}), 2.19-2.36(\mathrm{~m}, 3 \mathrm{H}), 1.82-2.03(\mathrm{~m}, 3 \mathrm{H}), 1.95(\mathrm{t}, J=2.8 \mathrm{~Hz}, 1 \mathrm{H})$, $1.62-1.70(\mathrm{~m}, 1 \mathrm{H}), 1.50-1.59(\mathrm{~m}, 1 \mathrm{H}), 1.38-1.49(\mathrm{~m}, 1 \mathrm{H}), 1.43(\mathrm{~s}, 3 \mathrm{H}), 1.42(\mathrm{~s}, 3 \mathrm{H}), 1.23(\mathrm{~d}$, $J=6.8 \mathrm{~Hz}, 3 \mathrm{H}), 1.15-1.28(\mathrm{~m}, 2 \mathrm{H}), 1.06(\mathrm{~d}, J=6.4 \mathrm{~Hz}, 3 \mathrm{H}), 0.96(\mathrm{t}, J=7.6 \mathrm{~Hz}, 9 \mathrm{H}), 0.57-0.73$ $(\mathrm{m}, 6 \mathrm{H}) ;{ }^{13} \mathrm{C}$ NMR $\left(100 \mathrm{MHz}, \mathrm{CDCl}_{3}\right) \delta 174.2,138.4,135.5,131.3,127.6,125.7,109.0,83.0$, 82.9, 82.3, 79.9, 78.5, 77.2, 75.1, 69.3, 44.2, 33.2, 32.0, 29.6, 28.6, 27.2, 27.1, 27.0, 22.5, 16.9, 15.6, 7.1, 5.6; IR (neat) 3310, 2950, 2874, 1732, 1378, 1237, 1170, $1053 \mathrm{~cm}^{-1}$; HRMS (ES+) $\mathrm{m} / z$ for $\mathrm{C}_{33} \mathrm{H}_{52} \mathrm{O}_{6} \mathrm{SiNa}[\mathrm{M}+\mathrm{Na}]^{+}$calcd 595.3431, found 595.3442.

Stannylalumination-protonolysis of the macrocycle alkyne was accomplished using a procedure analogous to that outlined for the conversion of $\mathbf{4 0}$ to $\mathbf{4 1}$ : macrocycle alkyne from the preceding step 17 (44 mg, $0.077 \mathrm{mmol})$, THF (1 mL), Bu $3 \mathrm{Sn}^{-\mathrm{AlEt}_{2}}(1.1 \mathrm{~mL}$ of the $0.41 \mathrm{M}$ solution, $0.45 \mathrm{mmol})$ and $\mathrm{CuCN}(2 \mathrm{mg}, 0.022 \mathrm{mmol})$ were used. Purification of the crude product by flash column chromatography afforded the vinylstannane $(38 \mathrm{mg}, 58 \%)$ as a colorless oil: $[\alpha]^{25} \mathrm{D}=-34.5^{\circ}\left(c 0.11, \mathrm{CHCl}_{3}\right) ;{ }^{1} \mathrm{H} \mathrm{NMR}\left(400 \mathrm{MHz}, \mathrm{CDCl}_{3}\right) \delta$ 6.15-6.26 (m, 
2H), 5.72 (app ddd, $J=3.6,9.6,15.2 \mathrm{~Hz}, 1 \mathrm{H}), 5.63\left(\mathrm{app} \mathrm{t},{ }^{3} J_{\mathrm{Sn}-\mathrm{H}}=70 \mathrm{~Hz}, 1 \mathrm{H}\right), 5.50-5.90(\mathrm{~m}$, $2 \mathrm{H}), 5.34(\mathrm{dd}, J=8.8,15.2 \mathrm{~Hz}, 1 \mathrm{H}), 5.14\left(\mathrm{dt}, \mathrm{J}=2.4 \mathrm{~Hz},{ }^{3} J_{\mathrm{Sn}-\mathrm{H}}=31.6 \mathrm{~Hz}, 1 \mathrm{H}\right), 4.50(\mathrm{~d}, J=$ $10.0 \mathrm{~Hz}, 1 \mathrm{H}), 4.02$ (app dt, $J=8.8,24.0 \mathrm{~Hz}, 2 \mathrm{H}), 3.75(\mathrm{~d}, J=8.8 \mathrm{~Hz}, 1 \mathrm{H}), 3.15-3.35(\mathrm{~m}, 3 \mathrm{H})$, $2.33(\mathrm{~d}, J=13.2 \mathrm{~Hz}, 2 \mathrm{H}), 1.82-2.06(\mathrm{~m}, 4 \mathrm{H}), 1.60-1.70(\mathrm{~m}, 1 \mathrm{H}), 1.40-1.57(\mathrm{~m}, 8 \mathrm{H}), 1.44(\mathrm{~s}$, $3 \mathrm{H}), 1.43(\mathrm{~s}, 3 \mathrm{H}), 1.25-1.36(\mathrm{~m}, 7 \mathrm{H}), 1.22(\mathrm{~d}, J=6.8 \mathrm{~Hz}, 3 \mathrm{H}), 1.18-1.24(\mathrm{~m}, 2 \mathrm{H}), 0.96(\mathrm{t}, J=$ $8.0 \mathrm{~Hz}, 9 \mathrm{H}), 0.93-0.99(\mathrm{~m}, 1 \mathrm{H}), 0.85-0.93(\mathrm{~m}, 14 \mathrm{H}), 0.81(\mathrm{~d}, J=6.4 \mathrm{~Hz}, 3 \mathrm{H}), 0.55-0.72(\mathrm{~m}$, $6 \mathrm{H}) ;{ }^{13} \mathrm{C} \mathrm{NMR}\left(100 \mathrm{MHz}, \mathrm{CDCl}_{3}\right) \delta 174.2,154.1,138.5,136.0,135.8,131.1,127.5,126.7$, 125.7, 109.0, 83.1, 82.4, 80.1, 79.6, 77.2, 75.0, 45.4, 44.4, 33.0, 32.1, 29.7, 29.2, 29.1, 28.5, 27.4, 27.2, 27.1, 16.9, 14.8, 13.7, 9.6, 7.2, 5.7; IR (neat) 2954, 2931, 2873, 1732, 1237, 1170, $1053 \mathrm{~cm}^{-1}$; HRMS (ES+) $\mathrm{m} / z$ for $\mathrm{C}_{45} \mathrm{H}_{80} \mathrm{O}_{6} \mathrm{SiSnNa}[\mathrm{M}+\mathrm{Na}]^{+}$calcd 887.4644, found 887.4655.

Iododestannylation of the vinylstannane intermediate was accomplished using a procedure analogous to that outlined for the conversion of $\mathbf{4 0}$ to $\mathbf{4 1}$ : vinylstannane from the preceding step (121 mg, $0.140 \mathrm{mmol})$, dichloromethane ( $2 \mathrm{~mL}$ ) and NIS (38 mg, $0.17 \mathrm{mmol})$ were used. The crude product was purified by flash column chromatography to afford 77 (94 $\mathrm{mg}, 96 \%$ ) as a colorless oil: $[\alpha]^{25} \mathrm{D}=-63^{\circ}\left(c 0.13, \mathrm{CHCl}_{3}\right) ;{ }^{1} \mathrm{H}$ NMR $\left(400 \mathrm{MHz}, \mathrm{CDCl}_{3}\right) \delta 6.15-6.28$ $(\mathrm{m}, 2 \mathrm{H}), 6.04(\mathrm{~s}, 1 \mathrm{H}), 5.73(\mathrm{~s}, 1 \mathrm{H}), 5.72(\mathrm{ddd}, J=4.0,10.4,14.8 \mathrm{~Hz}, 1 \mathrm{H}), 5.53$ (app dt, $J=$ $9.2,14.4 \mathrm{~Hz}, 2 \mathrm{H}), 5.33(\mathrm{dd}, J=8.8,15.2 \mathrm{~Hz}, 1 \mathrm{H}), 4.56(\mathrm{~d}, J=10.0 \mathrm{~Hz}, 1 \mathrm{H}), 4.02$ (app dt, $J=$ 8.8, 28.4 Hz, 2H), $3.73(\mathrm{~d}, J=8.8 \mathrm{~Hz}, 1 \mathrm{H}), 3.20-3.37(\mathrm{~m}, 3 \mathrm{H}), 2.28-2.48(\mathrm{~m}, 3 \mathrm{H}), 2.00$ (dd, $J=10.0,13.6 \mathrm{~Hz}, 3 \mathrm{H}), 1.82-1.95(\mathrm{~m}, 2 \mathrm{H}), 1.62-1.70(\mathrm{~m}, 1 \mathrm{H}), 1.49-1.58(\mathrm{~m}, 1 \mathrm{H}), 1.43(\mathrm{~s}, 3 \mathrm{H})$, $1.42(\mathrm{~s}, 3 \mathrm{H}), 1.31-1.46(\mathrm{~m}, 2 \mathrm{H}), 1.22(\mathrm{~d}, J=6.8 \mathrm{~Hz}, 3 \mathrm{H}), 1.16-1.20(\mathrm{~m}, 1 \mathrm{H}), 0.98(\mathrm{t}, J=8.0$ $\mathrm{Hz}, 9 \mathrm{H}), 0.85(\mathrm{~d}, J=6.4 \mathrm{~Hz}, 3 \mathrm{H}), 0.56-0.75(\mathrm{~m}, 6 \mathrm{H}) ;{ }^{13} \mathrm{C} \mathrm{NMR}\left(100 \mathrm{MHz}, \mathrm{CDCl}_{3}\right) \delta 174.4$, 138.3, 135.9, 135.5, 131.3, 127.7, 127.1, 125.7, 111.4, 109.0, 83.1, 82.3, 80.0, 78.6, 77.2, 75.0, 48.0, 44.1, 32.7, 32.0, 29.5, 28.5, 27.2, 27.0, 16.9, 14.5, 7.2, 5.7; IR (neat) 2950, 2874, 1731, $1378,1237,1170,1053 \mathrm{~cm}^{-1}$; HRMS (ES+) $\mathrm{m} / z$ for $\mathrm{C}_{33} \mathrm{H}_{53} \mathrm{IO}_{6} \mathrm{SiNa}[\mathrm{M}+\mathrm{Na}]^{+}$calcd 723.2554, found 723.2559 .

\section{Amphidinolide E (1)}

Deprotection of $\mathbf{7 7}$ was accomplished using a procedure analogous to that outlined for the conversion of 41 to 2 : vinyl iodide 77 ( $15 \mathrm{mg}, 0.021 \mathrm{mmol})$ and AcOH, THF and water (4/1/1) $(0.5 \mathrm{~mL})$ were used. The crude product was purified by flash column chromatography to afford only the $\mathrm{C} 18$ lactone $(9 \mathrm{mg}, 77 \%)$ as a colorless oil: $[\alpha]^{25} \mathrm{D}=-14.2^{\circ}\left(c 0.12, \mathrm{CHCl}_{3}\right) ;{ }^{1} \mathrm{H}$ NMR $\left(400 \mathrm{MHz}, \mathrm{CDCl}_{3}\right) \delta 6.10-6.28(\mathrm{~m}, 2 \mathrm{H}), 6.05(\mathrm{~s}, 1 \mathrm{H}), 5.74(\mathrm{~s}, 1 \mathrm{H}), 5.50-5.69(\mathrm{~m}, 3 \mathrm{H}), 5.26(\mathrm{dd}$, $J=8.0,15.2 \mathrm{~Hz}, 1 \mathrm{H}), 4.68(\mathrm{~d}, J=9.2 \mathrm{~Hz}, 1 \mathrm{H}), 3.91(\mathrm{app} \mathrm{dt}, J=8.8,28.8 \mathrm{~Hz}, 2 \mathrm{H}), 3.68-3.74$ $(\mathrm{m}, 1 \mathrm{H}), 3.55($ app q, $J=7.6 \mathrm{~Hz}, 1 \mathrm{H}), 3.36-3.44(\mathrm{~m}, 1 \mathrm{H}), 3.22-3.31(\mathrm{~m}, 1 \mathrm{H}), 3.35-2.57(\mathrm{~m}$, $4 \mathrm{H}), 2.23-2.31(\mathrm{~m}, 1 \mathrm{H}), 2.05(\mathrm{dd}, J=10.0,14.0 \mathrm{~Hz}, 1 \mathrm{H}), 1.72-1.93(\mathrm{~m}, 3 \mathrm{H}), 1.29-1.62(\mathrm{~m}$, $5 \mathrm{H}), 1.25(\mathrm{~d}, J=6.8 \mathrm{~Hz}, 3 \mathrm{H}), 0.93(\mathrm{~d}, J=6.8 \mathrm{~Hz}, 3 \mathrm{H}) ;{ }^{13} \mathrm{C} \mathrm{NMR}\left(100 \mathrm{MHz}, \mathrm{CDCl}_{3}\right) \delta 174.4$, 135.0, 134.9, 134.1, 131.5, 129.5, 127.3, 110.7, 79.8, 78.1, 77.6, 77.2, 77.1, 76.6, 73.3, 48.4, 44.0, 33.2, 32.5, 29.9, 29.0, 27.1, 17.5, 14.7; IR (neat) 3428, 2932, 2873, 1729, 1167, 990 $\mathrm{cm}^{-1}$; HRMS (ES+) $\mathrm{m} / z$ for $\mathrm{C}_{24} \mathrm{H}_{35} \mathrm{IO}_{6} \mathrm{Na}[\mathrm{M}+\mathrm{Na}]^{+}$calcd 569.1376 , found 569.1369 .

Stille coupling of the $\mathrm{C} 18$ lactone from above was accomplished using a procedure analogous to that outlined for the conversion of $\mathbf{4 1}$ to $\mathbf{2}$ : vinyl iodide lactone from the preceding step (20 $\mathrm{mg}, 0.037 \mathrm{mmol}), \mathrm{CuCl}(20 \mathrm{mg}, 0.201 \mathrm{mmol})$, THF $(0.5 \mathrm{~mL})$, vinylstannane 6 (68 $\mathrm{mg}, 0.183$ $\mathrm{mmol})$ and $\mathrm{Pd}\left(\mathrm{PPh}_{3}\right)_{4}(8.5 \mathrm{mg}, 0.00732 \mathrm{mmol})$ were used. The crude product was purified by flash column chromatography using $10 \%$ methanol/chloroform to afford material that was still contaminated with an organotin impurity. The stannane impurity was removed by HPLC purification with $100 \%$ ethyl acetate eluent on a normal phase, Varian Dynamax Microsorb $60-8 \mathrm{Si}, 250 \times 21.4 \mathrm{~mm}$ column. The retention time for amphidinolide $\mathrm{E}$ was $9.5 \mathrm{~min}$. The flow rate was $18 \mathrm{~mL} / \mathrm{min}$. Amphidinolide $\mathrm{E}$ was detected using $\mathrm{UV}$ absorption $(\lambda=254 \mathrm{~nm}$ and 280 $\mathrm{nm})$ and RI detection. Using the above conditions $10.6 \mathrm{mg} \mathrm{(59 \% )}$ of pure synthetic amphidinolide E was isolated: $[\alpha]^{25} \mathrm{D}=-86^{\circ}\left(c 0.08, \mathrm{CHCl}_{3}\right) ;{ }^{1} \mathrm{H} \mathrm{NMR}\left(400 \mathrm{MHz}, \mathrm{CDCl}_{3}\right) \delta$ 
6.10-6.28 (m, 2H) (H4 and H5), 6.05 (d, $J=15.2 \mathrm{~Hz}, 1 \mathrm{H})(\mathrm{H} 22), 5.58-5.75(\mathrm{~m}, 3 \mathrm{H})(\mathrm{H} 3, \mathrm{H} 10$, H23), 5.53 (dd, J = 8.8, 14.4, 1H) (H6), 5.27 (dd, $J=7.6,14.4$ Hz, 1H) (H9), 4.98 (s, 1H) (H29), 4.87 (s, 1H) (H29), 4.75 (s, 1H) (H26), $4.71(\mathrm{~s}, 1 \mathrm{H})(\mathrm{H} 26), 4.66$ (d, J = 9.2 Hz, 1H) (H18), 3.95 (t, $J=8.4 \mathrm{~Hz}, 1 \mathrm{H})(\mathrm{H} 8), 3.89(\mathrm{t}, J=8.8 \mathrm{~Hz}, 1 \mathrm{H})(\mathrm{H} 7), 3.68-3.74(\mathrm{~m}, 1 \mathrm{H})(\mathrm{H} 17)$, $3.52-3.60(\mathrm{~m}, 1 \mathrm{H})(\mathrm{H} 16), 3.36-3.45(\mathrm{~m}, 1 \mathrm{H})(\mathrm{H} 13), 3.21-3.30(\mathrm{~m}, 1 \mathrm{H})(\mathrm{H} 2), 2.71-2.84(\mathrm{~m}, 2 \mathrm{H})$ (H24), 2.20-2.45 (m, 6H) (H20a, H19, H11a and -OH x 3), 1.75-1.94 (m, 3H) (H11b, H12a, $\mathrm{H} 20 \mathrm{~b}), 1.72$ (s, 3H) (H27), 1.51-1.68 (m, 1H) (H15a, overlapping w/water), 1.21-1.51 (m, 4H) (H12b, H14a, H14b, H15b), 1.25 (d, $J=6.8 \mathrm{~Hz}, 3 \mathrm{H})(\mathrm{H} 30), 0.92$ (d, $J=6.8 \mathrm{~Hz}, 3 \mathrm{H})$ $(\mathrm{H} 29) ;{ }^{13} \mathrm{C}$ NMR $\left(100 \mathrm{MHz}, \mathrm{CDCl}_{3}\right) \delta 174.4,144.4,144.0,135.1,135.0,134.1,133.3,131.4$, 131.4, 129.4, 127.9, 115.7, 110.7, 79.9, 78.3, 78.0, 77.6, 76.7 (overlapping w/chloroform), 73.2, 44.1, 41.2, 36.0, 32.6, 32.3, 29.9, 28.9, 27.1, 22.5, 17.5, 15.3; IR (neat) 3439, 2929, 1731, 1454, 1168, $990 \mathrm{~cm}^{-1}$; HRMS (ES+) $\mathrm{m} / z$ for $\mathrm{C}_{30} \mathrm{H}_{44} \mathrm{O}_{6} \mathrm{Na}[\mathrm{M}+\mathrm{Na}]^{+}$calcd 523.3036, found 523.3038 .

\section{9-epi-Amphidinolide E (3) series}

Tricarbonyl[(E)-(2S,3R)-2-Methyl-hexa-3,5-dienoic acid (1R,2S)-1-((R)-\{(2S,5S)-5-[(E)-4-((4R, 5R)-2,2-dimethyl-5-vinyl-[1,3]dioxolan-4-yl)-but-3-enyl]-tetrahydro-furan-2-yl\}triethylsilanyloxy-methyl)-2-methyl-pent-4-ynyl ester]iron (78)

Esterification of alcohol 67 with acid 9 was accomplished using a procedure analogous to that outlined for 39: alcohol $67(0.34 \mathrm{~g}, 0.68 \mathrm{mmol})$, acid $9(0.29 \mathrm{~g}, 1.09 \mathrm{mmol})$, triethylamine $(0.35$ $\mathrm{mL}, 2.4 \mathrm{mmol}), \mathrm{DMAP}(83 \mathrm{mg}, 0.68 \mathrm{mmol}), \mathrm{THF}(1.5 \mathrm{~mL})$ and 2,4,6-trichlorobenzoyl chloride $(0.17 \mathrm{~mL}, 1.09 \mathrm{mmol})$ were used. Purification of the crude product by flash column chromatography afforded $78(0.501 \mathrm{~g}, 99 \%)$ as a colorless oil: $[\alpha]^{25}=+13^{\circ}(c 0.25$, $\left.\mathrm{CHCl}_{3}\right) ;{ }^{1} \mathrm{H} \mathrm{NMR}\left(400 \mathrm{MHz}, \mathrm{CDCl}_{3}\right) \delta 5.74-5.84(\mathrm{~m}, 2 \mathrm{H}), 5.39-5.49(\mathrm{~m}, 2 \mathrm{H}), 5.34(\mathrm{~d}, J=$ $17.6 \mathrm{~Hz}, 1 \mathrm{H}), 5.20-5.28(\mathrm{~m}, 2 \mathrm{H}), 4.86(\mathrm{~d}, J=8.4 \mathrm{~Hz}, 1 \mathrm{H}), 4.02-4.09(\mathrm{~m}, 2 \mathrm{H}), 3.68-3.78(\mathrm{~m}$, 2H), 3.61-3.68 (m, 1H), 2.06-2.40 (m, 6H), 1.99 (t, $J=2.4 \mathrm{~Hz}, 1 \mathrm{H}), 1.72-1.90(\mathrm{~m}, 3 \mathrm{H})$, $1.46-1.70(\mathrm{~m}, 3 \mathrm{H}), 1.45(\mathrm{~s}, 3 \mathrm{H}), 1.44(\mathrm{~s}, 3 \mathrm{H}), 1.32(\mathrm{~d}, J=6.8 \mathrm{~Hz}, 3 \mathrm{H}), 0.89-1.02(\mathrm{~m}, 13 \mathrm{H})$, $0.60-0.77(\mathrm{~m}, 6 \mathrm{H}), 0.33$ (bdd, $J=1.6,8.4 \mathrm{~Hz}, 1 \mathrm{H}) ;{ }^{13} \mathrm{C} \mathrm{NMR}\left(100 \mathrm{MHz}, \mathrm{CDCl}_{3}\right) \delta 211.3$, 173.8, 136.2, 134.4, 126.1, 118.6, 108.9, 87.5, 82.4, 82.3, 81.7, 80.7, 78.7, 77.1, 75.3, 70.4, $64.1,44.6,40.5,35.3,32.9,31.0,29.3,27.8,27.2,27.1,22.6,19.4,15.9,7.2,5.5$; IR (neat) $3311,2936,2049,1979,1731 \mathrm{~cm}^{-1}$; HRMS (ESI-TOF+) $m / z$ for $\mathrm{C}_{38} \mathrm{H}_{56} \mathrm{FeO}_{9} \mathrm{SiH}[\mathrm{M}+\mathrm{H}]^{+}$ calcd 741.3116, found 741.3101.

(4E,11E,13E)-(1S,6R,10R,15R,18R,19R,20S)-18-((S)-3-lodo-1-methyl-but-3-enyl)-8,8,15trimethyl-19-triethylsilanyloxy-7,9,17,23-tetraoxa-tricyclo[18.2.1.06,10] tricosa-4,11,13trien-16-one (79)

The oxidative decomplexation of 78 was accomplished using a procedure analogous to that outlined for the conversion of 39 to 40 : ester $78(0.45 \mathrm{~g}, 0.60 \mathrm{mmol})$, acetone $(6 \mathrm{~mL})$ and cerium ammonium nitrate (CAN) $(0.66 \mathrm{~g}, 1.2 \mathrm{mmol})$ were used. The crude product was purified by flash column chromatography to afford the polyene product $(0.30 \mathrm{~g}, 82 \%)$ as a colorless oil: $[\alpha]^{25} \mathrm{D}=-7.3^{\circ}\left(c 1.6, \mathrm{CHCl}_{3}\right) ;{ }^{1} \mathrm{H} \mathrm{NMR}\left(400 \mathrm{MHz}, \mathrm{CDCl}_{3}\right) \delta 6.31(\mathrm{dt}, J=10.4,16.8,1 \mathrm{H})$, $6.15(\mathrm{dd}, J=10.8,15.6 \mathrm{~Hz}, 1 \mathrm{H}), 5.74-5.84(\mathrm{~m}, 3 \mathrm{H}), 5.43$ (bdd, $J=5.6,14.4 \mathrm{~Hz}, 1 \mathrm{H}), 5.33$ (d, $J=17.2 \mathrm{~Hz}, 1 \mathrm{H}), 5.23(\mathrm{~d}, J=10.4 \mathrm{~Hz}, 1 \mathrm{H}), 5.17(\mathrm{~d}, J=16.4,1 \mathrm{H}), 5.06(\mathrm{~d}, J=10.0 \mathrm{~Hz}, 1 \mathrm{H})$, 4.86 (dd, $J=2.4,8.0 \mathrm{~Hz}, 1 \mathrm{H}), 4.02-4.09$ (m, 2H), 3.60-3.77 (m, 1H), 3.22 (quint., $J=7.2 \mathrm{~Hz}$, $1 \mathrm{H}), 2.31-2.41(\mathrm{~m}, 1 \mathrm{H}), 2.04-2.26(\mathrm{~m}, 4 \mathrm{H}), 1.98(\mathrm{t}, J=2.8 \mathrm{~Hz}, 1 \mathrm{H}), 1.72-1.91(\mathrm{~m}, 2 \mathrm{H})$, $1.58-1.69(\mathrm{~m}, 1 \mathrm{H}), 1.39-1.57(\mathrm{~m}, 3 \mathrm{H}), 1.44(\mathrm{~s}, 3 \mathrm{H}), 1.44(\mathrm{~s}, 3 \mathrm{H}), 1.30(\mathrm{~d}, J=6.8 \mathrm{~Hz}, 3 \mathrm{H}), 0.99$ $(\mathrm{d}, J=6.8 \mathrm{~Hz}, 3 \mathrm{H}), 0.96(\mathrm{t}, J=8.0 \mathrm{~Hz}, 9 \mathrm{H}), 0.57-0.74(\mathrm{~m}, 6 \mathrm{H}) ;{ }^{13} \mathrm{C} \mathrm{NMR}(100 \mathrm{MHz}$, $\left.\mathrm{CDCl}_{3}\right) \delta 173.8,136.5,136.2,134.4,132.7,132.3,126.0,118.5,117.1,108.9,82.3,82.3,81.9$, 80.5, 78.6, 77.0, 75.2 , 70.2, 43.0, 35.3, 33.0, 31.0, 29.3, 27.6, 27.1, 27.0, 22.7, 17.2 , 15.7, 7.1, 5.4; IR (neat) 3309, 2953, 1983, 1734, 1239, 1169, $1054 \mathrm{~cm}^{-1}$; HRMS (ESI-TOF+) $\mathrm{m} / \mathrm{z}$ for $\mathrm{C}_{35} \mathrm{H}_{56} \mathrm{O}_{6} \mathrm{SiNa}[\mathrm{M}+\mathrm{Na}]^{+}$calcd 623.3744, found 623.3728 . 
Ring closing metathesis of the polyene was accomplished using a procedure analogous to that outlined for the conversion of $\mathbf{3 9}$ to 40: polyene from the preceding step ( $124 \mathrm{mg}, 0.206 \mathrm{mmol}$ ), dichloromethane $(206 \mathrm{~mL})$ and Grubbs' first generation catalyst $(17 \mathrm{mg}, 0.021 \mathrm{mmol})$ were used. The crude product was purified by flash column chromatography to afford the macrocycle product $(67 \mathrm{mg}, 57 \%)$ as a colorless oil. In addition, an inseparable mixture of products thought to arrive by enyne metathesis $(6 \mathrm{mg}, 5 \%)$ was also isolated. Spectroscopic data for the macrocycle product: $[\alpha]^{25} \mathrm{D}=-44^{\circ}\left(c 0.25, \mathrm{CHCl}_{3}\right) ;{ }^{1} \mathrm{H} \mathrm{NMR}\left(400 \mathrm{MHz}, \mathrm{CDCl}_{3}\right) \delta 6.14-6.26$ $(\mathrm{m}, 2 \mathrm{H}), 5.71(\mathrm{ddd}, J=3.2,9.6,14.4 \mathrm{~Hz}, 1 \mathrm{H}), 5.48-5.58(\mathrm{~m}, 2 \mathrm{H}), 5.32(\mathrm{dd}, J=8.8,15.2 \mathrm{~Hz}$, $1 \mathrm{H}), 4.76(\mathrm{~d}, J=8.0 \mathrm{~Hz}, 1 \mathrm{H}), 4.01$ (app dt, $J=8.4,25.2 \mathrm{~Hz}, 2 \mathrm{H}), 3.73(\mathrm{~d}, J=8.4 \mathrm{~Hz}, 1 \mathrm{H})$, 3.18-3.34 (m, 3H), 2.15-2.36 (m, 4H), $1.98(\mathrm{t}, J=2.0 \mathrm{~Hz}, 1 \mathrm{H}), 1.82-1.96(\mathrm{~m}, 2 \mathrm{H}), 1.61-1.70$ $(\mathrm{m}, 1 \mathrm{H}), 1.48-1.57(\mathrm{~m}, 1 \mathrm{H}), 1.38-1.48(\mathrm{~m}, 1 \mathrm{H}), 1.42(\mathrm{~s}, 3 \mathrm{H}), 1.42(\mathrm{~s}, 3 \mathrm{H}), 1.10-1.28(\mathrm{~m}, 2 \mathrm{H})$, $1.22(\mathrm{~d}, J=6.8 \mathrm{~Hz}, 3 \mathrm{H}), 0.98(\mathrm{~d}, J=6.4 \mathrm{~Hz}, 3 \mathrm{H}), 0.95(\mathrm{t}, J=8.0 \mathrm{~Hz}, 9 \mathrm{H}), 0.54-0.72(\mathrm{~m}$, $6 \mathrm{H}) ;{ }^{13} \mathrm{C}$ NMR $\left(100 \mathrm{MHz}, \mathrm{CDCl}_{3}\right) \delta 174.2,138.5,136.1,135.8,131.3,127.7,125.8,109.1$, 83.2, 82.5, 81.4, 80.1, 77.9, 75.1, 70.4, 44.4, 32.1, 29.6, 28.7, 27.3, 27.2, 27.1, 22.2, 17.0, 16.0, 7.3, 5.8; IR (neat) $3310,2935,1732,1237,1170 \mathrm{~cm}^{-1}$; HRMS (ESI-TOF+) $\mathrm{m} / \mathrm{z}$ for $\mathrm{C}_{33} \mathrm{H}_{52} \mathrm{O}_{6} \mathrm{SiH}[\mathrm{M}+\mathrm{H}]^{+}$calcd 573.3606, found 573.3596.

Stannylalumination-protonolysis of the macrocycle alkyne was accomplished using a procedure analogous to that outlined for the conversion of $\mathbf{4 0}$ to $\mathbf{4 1}$ : macrocycle alkyne from above ( $32 \mathrm{mg}, 0.056 \mathrm{mmol})$, THF ( $1 \mathrm{~mL}), \mathrm{Bu}_{3} \mathrm{Sn}_{-} \mathrm{AlEt}_{2}(0.80 \mathrm{~mL}$ of the $0.42 \mathrm{M}$ solution, 0.34 $\mathrm{mmol})$ and $\mathrm{CuCN}(1 \mathrm{mg}, 0.011 \mathrm{mmol})$ were used. Purification of the crude product by flash column chromatography afforded the vinylstannane $(31 \mathrm{mg}, 64 \%)$ as a colorless oil: $[\alpha]^{25} \mathrm{D}=$ $-119^{\circ}\left(c \mathrm{c} 0.10, \mathrm{CHCl}_{3}\right) ;{ }^{1} \mathrm{H}$ NMR $\left(400 \mathrm{MHz}, \mathrm{CDCl}_{3}\right) \delta$ 6.15-6.28 (m, 2H), 5.67-5.76 (m, 2H), 5.53 (app dd, $J=9.6,14.4 \mathrm{~Hz}, 2 \mathrm{H}), 5.33(\mathrm{dd}, J=8.8,15.6 \mathrm{~Hz}, 1 \mathrm{H}), 5.20\left(\mathrm{t},{ }^{3} J_{S n-H}=32.0 \mathrm{~Hz}\right.$, $1 \mathrm{H}$ ), $4.57(\mathrm{~d}, J=7.6 \mathrm{~Hz}, 1 \mathrm{H}), 4.02$ (app dt, $J=8.8,23.2 \mathrm{~Hz}, 2 \mathrm{H}$ ), 3.72 (app q, $J=12.4 \mathrm{~Hz}$, $1 \mathrm{H}), 3.19-3.36(\mathrm{~m}, 3 \mathrm{H}), 2.49(\mathrm{bd}, J=13.6 \mathrm{~Hz}, 1 \mathrm{H}), 2.28-2.36(\mathrm{~m}, 1 \mathrm{H}), 2.10-2.20(\mathrm{~m}, 1 \mathrm{H})$, $1.83-2.24(\mathrm{~m}, 3 \mathrm{H}), 1.56-1.71(\mathrm{~m}, 1 \mathrm{H}), 1.40-1.56(\mathrm{~m}, 9 \mathrm{H}), 1.43(\mathrm{~s}, 3 \mathrm{H}), 1.43(\mathrm{~s}, 3 \mathrm{H}), 1.25-1.36$ $(\mathrm{m}, 7 \mathrm{H}), 1.22(\mathrm{~d}, J=6.8 \mathrm{~Hz}, 3 \mathrm{H}), 0.96(\mathrm{t}, J=7.6 \mathrm{~Hz}, 9 \mathrm{H}), 0.85-0.92(\mathrm{~m}, 14 \mathrm{H}), 0.81(\mathrm{~d}, J=6.8$ $\mathrm{Hz}, 3 \mathrm{H}), 0.59-0.69(\mathrm{~m}, 6 \mathrm{H}) ;{ }^{13} \mathrm{C}$ NMR $\left(100 \mathrm{MHz}, \mathrm{CDCl}_{3}\right) \delta 174.3,152.9,138.6,136.2,136.0$, 131.3, 127.6, 126.6, 125.8, 109.1, 83.2, 82.5, 80.3, 80.0, 77.5, 75.9, 44.4, 44.0, 33.1, 32.3, 29.7, 29.4, 29.3, 28.7, 27.5, 27.3, 27.2, 17.1, 15.7, 13.8, 9.6, 7.4, 5.8; IR (neat) 2955, 1732, 1171, $1054 \mathrm{~cm}^{-1}$; HRMS (ESI-TOF+) $\mathrm{m} / z$ for $\mathrm{C}_{45} \mathrm{H}_{80} \mathrm{O}_{6} \mathrm{SiSnH}[\mathrm{M}+\mathrm{H}]^{+}$calcd 865.4819, found 865.4825.

Iododestannylation of vinylstannane was accomplished using a procedure analogous to that outlined for the conversion of $\mathbf{4 0}$ to $\mathbf{4 1}$ : vinylstannane from the preceding step (54 mg, 0.063 $\mathrm{mmol})$, dichloromethane $(2 \mathrm{~mL}$ ) and NIS (17 $\mathrm{mg}, 0.075 \mathrm{mmol})$ were used. The crude product was purified by flash column chromatography to afford $79(34 \mathrm{mg}, 77 \%)$ as a colorless oil: $[\alpha]^{25}{ }_{\mathrm{D}}=-110^{\circ}\left(c 0.11, \mathrm{CHCl}_{3}\right) ;{ }^{1} \mathrm{H}$ NMR $\left(400 \mathrm{MHz}, \mathrm{CDCl}_{3}\right) \delta$ 6.15-6.28 (m, 2H), $6.09(\mathrm{~s}$, $1 \mathrm{H}), 5.76(\mathrm{~s}, 1 \mathrm{H}), 5.72(\mathrm{ddd}, J=3.6,9.6,15.2 \mathrm{~Hz}, 1 \mathrm{H}), 5.48-5.58(\mathrm{~m}, 2 \mathrm{H}), 5.33(\mathrm{dd}, J=8.8$, $15.2 \mathrm{~Hz}, 1 \mathrm{H}), 4.60(\mathrm{~d}, J=7.6 \mathrm{~Hz}, 1 \mathrm{H}), 4.02(\mathrm{app} \mathrm{dt}, J=8.4,25.2 \mathrm{~Hz}, 2 \mathrm{H}), 3.68(\mathrm{~d}, J=8.8 \mathrm{~Hz}$, $1 \mathrm{H}), 3.30-3.38(\mathrm{~m}, 1 \mathrm{H}), 3.19-3.30(\mathrm{~m}, 2 \mathrm{H}), 2.63(\mathrm{dd}, J=4.4,13.6 \mathrm{~Hz}, 1 \mathrm{H}), 2.26-2.39(\mathrm{~m}, 2 \mathrm{H})$, $2.12(\mathrm{dd}, J=9.2,13.6 \mathrm{~Hz}, 1 \mathrm{H}), 1.84-2.10(\mathrm{~m}, 2 \mathrm{H}), 1.36-1.55(\mathrm{~m}, 2 \mathrm{H}), 1.43(\mathrm{~s}, 3 \mathrm{H}), 1.43(\mathrm{~s}$, $3 \mathrm{H}), 1.11-1.27(\mathrm{~m}, 3 \mathrm{H}), 1.23(\mathrm{~d}, J=7.2 \mathrm{~Hz}, 3 \mathrm{H}), 0.97(\mathrm{t}, J=7.6 \mathrm{~Hz}, 9 \mathrm{H}), 0.84(\mathrm{~d}, J=6.8 \mathrm{~Hz}$, $3 \mathrm{H}), 0.60-0.71(\mathrm{~m}, 6 \mathrm{H}) ;{ }^{13} \mathrm{C} \mathrm{NMR}\left(100 \mathrm{MHz}, \mathrm{CDCl}_{3}\right) \delta 174.3,138.6,136.2,135.7,131.5$, 127.7, 127.5, 125.8, 110.7, 109.2, 83.1, 82.5, 80.1, 77.7, 77.6, 75.9, 48.5, 44.3, 33.3, 32.2, 29.6, 28.7, 27.3, 27.2, 27.2, 17.0, 15.0, 7.4, 5.8; IR (neat) 2981, 1731, 1377, 1170, $1053 \mathrm{~cm}^{-1}$; HRMS (ESI-TOF+) $m / z$ for $\mathrm{C}_{33} \mathrm{H}_{53} \mathrm{IO}_{6} \mathrm{SiH}[\mathrm{M}+\mathrm{H}]^{+}$calcd 701.2729, found 701.2723.

\section{9-epi-amphidinolide E (3)}

Deprotection of $\mathbf{7 9}$ was accomplished using a procedure analogous to that outlined for the conversion of 41 to 2: vinyl iodide $\mathbf{7 9}$ (34 mg, $0.049 \mathrm{mmol}$ ) and AcOH, THF and water (4/1/1) 
$(1 \mathrm{~mL})$ were used. The crude product was purified by flash column chromatography to afford only the $\mathrm{C} 18$ lactone $(24 \mathrm{mg}, 90 \%)$ as a colorless oil: $[\alpha]^{25} \mathrm{D}=-90^{\circ}\left(c 0.18, \mathrm{CHCl}_{3}\right) ;{ }^{1} \mathrm{H} \mathrm{NMR}$ $\left(400 \mathrm{MHz}, \mathrm{CDCl}_{3}\right) \delta 6.10-6.26(\mathrm{~m}, 2 \mathrm{H}), 6.08(\mathrm{~s}, 1 \mathrm{H}), 5.76(\mathrm{~s}, 1 \mathrm{H}), 5.49-5.69(\mathrm{~m}, 3 \mathrm{H}), 5.26$ $(\mathrm{dd}, J=8.0,15.2 \mathrm{~Hz}, 1 \mathrm{H}), 4.75(\mathrm{~d}, J=8.0 \mathrm{~Hz}, 1 \mathrm{H}), 3.91(\operatorname{app~dt}, J=8.8,26.0 \mathrm{~Hz}, 2 \mathrm{H}), 3.63-3.68$ $(\mathrm{m}, 1 \mathrm{H}), 3.58-3.60(\mathrm{~m}, 1 \mathrm{H}), 3.34-3.46(\mathrm{~m}, 1 \mathrm{H}), 3.21-3.30(\mathrm{~m}, 1 \mathrm{H}), 2.66(\mathrm{dd}, J=3.6,13.6 \mathrm{~Hz}$, $1 \mathrm{H}), 2.33-2.50(\mathrm{~m}, 4 \mathrm{H}), 2.20-2.29(\mathrm{~m}, 1 \mathrm{H}), 2.14(\mathrm{app} \mathrm{dd}, J=9.6,14.0 \mathrm{~Hz}, 1 \mathrm{H}), 1.70-1.93(\mathrm{~m}$, $3 \mathrm{H}), 1.54-1.64(\mathrm{~m}, 1 \mathrm{H}), 1.32-1.49(\mathrm{~m}, 3 \mathrm{H}), 1.26(\mathrm{~d}, J=6.4 \mathrm{~Hz}, 3 \mathrm{H}), 0.89$ (d, $J=6.8 \mathrm{~Hz}$, $3 \mathrm{H}) ;{ }^{13} \mathrm{C} \mathrm{NMR}\left(100 \mathrm{MHz}, \mathrm{CDCl}_{3}\right) \delta 174.2,135.0,134.3,131.6,131.6,129.6,127.6,110.5$, 80.0, 78.3, 77.7, 76.2, 73.4, 48.3, 44.1, 33.9, 32.9, 30.1, 29.1, 27.2, 17.6, 15.0; IR (neat) 3430, 2933, 1731, 1169, $990 \mathrm{~cm}^{-1}$; HRMS (ES+) $m / z$ for $\mathrm{C}_{24} \mathrm{H}_{35} \mathrm{IO}_{6} \mathrm{Na}[\mathrm{M}+\mathrm{Na}]^{+}$calcd 569.1376, found 569.1367.

Stille coupling of the $\mathrm{C} 18$ lactone from was accomplished using a procedure analogous to that outlined for the conversion of $\mathbf{4 1}$ to 2: vinyl iodide from above (24 mg, $0.044 \mathrm{mmol}), \mathrm{CuCl}$ (24 mg, $0.24 \mathrm{mmol})$, THF (0.5 mL), vinylstannane 6 (82 mg, $0.22 \mathrm{mmol})$ and $\mathrm{Pd}\left(\mathrm{PPh}_{3}\right)_{4}(10$ $\mathrm{mg}, 0.0087 \mathrm{mmol}$ ) were used. The crude product was purified by flash column chromatography using $10 \%$ methanol/chloroform to afford material that was still contaminated with an organotin impurity. The stannane impurity was removed by HPLC purification with $100 \%$ ethyl acetate eluent on a normal phase, Varian Dynamax Microsorb 60-8 Si, $250 \times 21.4 \mathrm{~mm}$ column. The retention time for 19-epi-amphidinolide $\mathrm{E}$ was $7.5 \mathrm{~min}$. The flow rate was $18 \mathrm{~mL} /$ min. 19-epi-Amphidinolide E was detected using UV absorption ( $\lambda=254 \mathrm{~nm}$ and $280 \mathrm{~nm})$ and RI detection. Using the above conditions $15 \mathrm{mg}(68 \%)$ of pure 19-epi-amphidinolide $\mathrm{E}(3)$ was isolated: $[\alpha]^{25} \mathrm{D}=-7.8^{\circ}\left(c 0.50, \mathrm{CHCl}_{3}\right) ;{ }^{1} \mathrm{H} \mathrm{NMR}\left(400 \mathrm{MHz}, \mathrm{CDCl}_{3}\right) \delta 6.10-6.28(\mathrm{~m}, 2 \mathrm{H})$, $6.05(\mathrm{~d}, J=15.6 \mathrm{~Hz}, 1 \mathrm{H}), 5.87(\mathrm{dt}, J=6.8,16.0 \mathrm{~Hz}, 1 \mathrm{H}), 5.49-5.70(\mathrm{~m}, 3 \mathrm{H}), 5.27$ (dd, $J=8.0$, $15.2 \mathrm{~Hz}, 1 \mathrm{H}), 4.98(\mathrm{~s}, 1 \mathrm{H}), 4.88(\mathrm{~s}, 1 \mathrm{H}), 4.73(\mathrm{~s}, 1 \mathrm{H}), 4.70(\mathrm{~s}, 1 \mathrm{H}), 4.68(\mathrm{~d}, J=10.4 \mathrm{~Hz}, 1 \mathrm{H})$, $3.95(\mathrm{t}, J=8.4 \mathrm{~Hz}, 1 \mathrm{H}), 3.88(\mathrm{t}, J=8.8 \mathrm{~Hz}, 1 \mathrm{H}), 3.78(\operatorname{app~q}, J=8.8 \mathrm{~Hz}, 1 \mathrm{H}), 3.35-3.44(\mathrm{~m}$, $1 \mathrm{H}), 3.21-3.31(\mathrm{~m}, 1 \mathrm{H}), 2.77(\mathrm{~d}, J=6.8 \mathrm{~Hz}, 2 \mathrm{H}), 2.62(\mathrm{dd}, J=3.6,13.2 \mathrm{~Hz}, 1 \mathrm{H}), 2.24-2.46$ $(\mathrm{m}, 5 \mathrm{H}), 1.74-1.94(\mathrm{~m}, 4 \mathrm{H}), 1.71(\mathrm{~s}, 3 \mathrm{H}), 1.53-1.64(\mathrm{~m}, 1 \mathrm{H}), 1.22-1.51(\mathrm{~m}, 3 \mathrm{H}), 1.25(\mathrm{~d}, J=$ $6.8 \mathrm{~Hz}, 3 \mathrm{H}), 0.81(\mathrm{~d}, J=6.4 \mathrm{~Hz}, 3 \mathrm{H}) ;{ }^{13} \mathrm{C} \mathrm{NMR}\left(100 \mathrm{MHz}, \mathrm{CDCl}_{3}\right) \delta 174.3,144.8,143.9$, $135.4,135.1,134.3,133.1,131.6,129.6,128.5,116.3,111.0,80.1,78.2,78.0,77.7,76.8,73.4$, 44.2, 41.6, 36.0, 32.5, 32.2, 30.0, 29.1, 27.1, 22.7, 17.5, 15.7; IR (neat) 3400, 2931, 1731, 1169, $989 \mathrm{~cm}^{-1}$; HRMS (ESI-TOF+) $\mathrm{m} / z$ for $\mathrm{C}_{30} \mathrm{H}_{44} \mathrm{O}_{6} \mathrm{Na}[\mathrm{M}+\mathrm{Na}]^{+}$calcd 523.3036, found 523.3024 .

\section{Acknowledgements}

This work is supported by a grant from the National Institutes of Health (GM38436). Preliminary studies at the University of Michigan were supported by GM38907. We thank Dr. Troy Ryba for assistance with the HPLC purification of amphidinolide E, and Prof. Jun-ichi Kobayashi for providing comparative spectroscopic data for the natural product.

\section{References}

1. For reviews on the amphidinolides: (a) Kobayashi J, Ishibashi M. Chem Rev 1993;93:1753. (b) Chakraborty TK, Das S. Curr Med Chem: Anti-Cancer Agents 2001;1:131. (c) Kobayashi J, Shimbo K, Kubota T, Tsuda M. Pure Appl Chem 2003;75:337. (d) Kobayashi J, Tsuda M. Nat Prod Rep 2004;21:77. [PubMed: 15039836]

2. (a) Lam HW, Pattenden G. Angew Chem, Int Ed Engl 2002;41:508. [PubMed: 12491395] (b) Maleczka RE Jr, Terrell LR, Geng F, Ward JS III. Org Lett 2002;4:2841. [PubMed: 12182569] (c) Trost BM, Chisholm JD, Wrobleski SJ, Jung M. J Am Chem Soc 2002;124:12420. [PubMed: 12381177] (d) Trost BM, Harrington PE. J Am Chem Soc 2004;126:5028. [PubMed: 15099060] (e) Trost BM, Wrobleski ST, Chisholm JD, Harrington PE, Jung M. J Am Chem Soc 2005;127:13589. [PubMed: 16190724] (f) Trost BM, Harrington PE, Chisholm JD, Wrobleski ST. J Am Chem Soc 2005; 127:13598. [PubMed: 16190725]

3. Williams DR, Kissel WS. J Am Chem Soc 1998;120:11198. 
4. Williams DR, Meyer KG. J Am Chem Soc 2001;123:765. [PubMed: 11456603]

5. (a) Williams DR, Myers BJ, Mi L. Org Lett 2000;2:945. [PubMed: 10768193] (b) Trost BM, Papillon JPN. J Am Chem Soc 2004;126:13618. [PubMed: 15493910] (c) Trost BM, Papillon JPN, Nussbaumer T. J Am Chem Soc 2005;127:17921. [PubMed: 16351124]

6. (a) Fürstner A, Aissa C, Riveiros R, Ragot J. Angew Chem, Int Ed Engl 2002;41:4763. [PubMed: 12481352] (b) Aissa C, Riveiros R, Ragot J, Fürstner A. J Am Chem Soc 2003;125:15512. [PubMed: 14664598] (c) Ghosh AK, Liu C. J Am Chem Soc 2003;125:2374. [PubMed: 12603108] (d) Ghosh AK, Liu C. Strategies Tactics Org Synth 2004;5:255. (e) Colby EA, O’Brien KC, Jamison TF. J Am Chem Soc 2004;126:998. [PubMed: 14746449] (f) Colby EA, O'Brien KC, Jamison TF. J Am Chem Soc 2005;127:4297. [PubMed: 15783211] (g) O’Brien KC, Colby EA, Jamison TF. Tetrahedron 2005;61:6243. (h) Deng LS, Huang XP, Zhao G. J Org Chem 2006;71:4625. [PubMed: 16749797]

7. (a) Ghosh AK, Gong G. J Am Chem Soc 2004;126:3704. [PubMed: 15038710] (b) Ghosh AK, Gong G. J Org Chem 2006;71:1085. [PubMed: 16438525]

8. Lepage O, Kattnig E, Fürstner A. J Am Chem Soc 2004;126:15970. [PubMed: 15584724]

9. Fürstner A, Kattnig E, Lepage O. J Am Chem Soc 2006;128:9194. [PubMed: 16834393]

10. (a) Kobayashi J, Ishibashi M, Murayama T, Takamatsu M, Iwamura M, Ohizumi Y, Sasaki T. J Org Chem 1990;55:3421. (b) Kubota T, Tsuda M, Kobayashi J. J Org Chem 2002;67:1651. [PubMed: 11871898]

11. Kim CH, An HJ, Shin WK, Yu W, Woo SK, Jung SK, Lee E. Angew Chem Int Ed 2006;45:8019.

12. Gurjar MK, Mohapatra S, Phalgune UD, Puranik VG, Mohapatra DK. Tetrahedron Lett 2004;45:7899.

13. Marshall JA, Schaaf G, Nolting A. Org Lett 2005;7:5331. [PubMed: 16268571]

14. (a) Micalizio GC, Roush WR. Org Lett 2000;2:461. [PubMed: 10814351] (b) Micalizio GC, Roush WR. Org Lett 2001;3:1949. [PubMed: 11405752] (c) Shotwell JB, Roush WR. Org Lett 2004;6:3865. [PubMed: 15469369] (d) Tinsley JM, Roush WR. J Am Chem Soc 2005;127:10818. [PubMed: 16076173] (e) Mertz E, Tinsley JM, Roush WR. J Org Chem 2005;70:8035. [PubMed: 16277325] (f) Tinsley JM, Mertz E, Chong PY, Rarig RAF, Roush WR. Org Lett 2005;7:4245. [PubMed: 16146398] (g) Lambert WT, Roush WR. Org Lett 2005;7:5501. [PubMed: 16288541]

15. For reviews of reactions of allylsilanes: (a) Masse CE, Panek JS. Chem Rev 1995;95:1293. (b) Chabaud L, James P, Landais Y. Eur J Org Chem 2004:3173.

16. (a) Panek JS, Yang M. J Am Chem Soc 1991;113:9868.Panek JS, Beresis R. J Org Chem 1993;58:809. (c) Beresis R, Panek JS. Bioorg Med Chem Lett 1993;3:1609. (d) Peng ZH, Woerpel KA. Organic Letters 2002;4:2945. [PubMed: 12182595] (e) Peng ZH, Woerpel KA. Org Lett 2001;3:675. [PubMed: 11259034] (f) Roberson CW, Woerpel KA. J Am Chem Soc 2002;124:11342. [PubMed: 12236749] (g) Peng ZH, Woerpel KA. J Am Chem Soc 2003;125:6018. [PubMed: 12785807]

17. Va P, Roush WR. J Am Chem Soc 2006;128:15960. [PubMed: 17165709]

18. Va P, Roush WR. Org Lett 2007;9:307. [PubMed: 17217291]

19. (a) Stille JK, Groh BL. J Am Chem Soc 1987;109:813. (b) Han X, Stoltz BM, Corey EJ. J Am Chem Soc 1999;121:7600.

20. Sarabia F, Sanchez-Ruiz A. J Org Chem 2005;70:9514. [PubMed: 16268627]

21. Johnson WS, Werthemann L, Bartlett WR, Brocksom TJ, Li TT, Faulkner DJ, Petersen MR. J Am Chem Soc 1970;92:741.

22. Roush WR, Koyama K, Curtin ML, Moriarty KJ. J Am Chem Soc 1996;118:7502.

23. (a) Roush WR, Halterman RL. J Am Chem Soc 1986;108:294. (b) Roush WR, Hoong LK, Palmer MAJ, Park JC. J Org Chem 1990;55:4109. (c) Roush WR, Hoong LK, Palmer MAJ, Straub JA, Palkowitz AD. J Org Chem 1990;55:4117. (d) Roush WR, Palkowitz AD, Ando K. J Am Chem Soc 1990;112:6348.

24. Schmid CR, Bradley DA. Synthesis 1992:587.

25. Parikh JR, Doering WvE. J Am Chem Soc 1967;89:5505.

26. Corey EJ, Fuchs PL. Tetrahedron Lett 1972;13:3769.

27. (a) Roush WR, Grover PT. Tetrahedron Lett 1990;31:7567. (b) Roush WR, Grover PT. Tetrahedron 1992;48:1981.

28. Dale JA, Mosher HS. J Am Chem Soc 1973;95:512. 
29. Hudrlik PF, Peterson D. J Am Chem Soc 1975;97:1464.

30. [3+2] annulations involving aldehydes with $\alpha$-chelating groups promoted by $\mathrm{SnCl} 4$ typically afford 2,5-trans-THF products.14a Since, aldehyde 5 has no $\alpha$-chelating group, we anticipated 2,5-cis product 24 to predominate.

31. Heitzman CL, Lambert WT, Mertz E, Shotwell JB, Tinsley JM, Va P, Roush WR. Org Lett 2005;7:2405. [PubMed: 15932209]

32. Horita K, Yoshioka T, Tanaka T, Oikawa Y, Yonemitsu O. Tetrahedron 1986;42:3021.

33. Acid $\mathbf{1 3}$ was synthesized by hydrolysis of the chiral auxiliary in oxazolidinone $\mathbf{3 6}$ (64\% yield).

34. Hikota M, Sakurai Y, Horita K, Yonemitsu O. Tetrahedron Lett 1990;31:6367.

35. For use of EDCI·MeI: Mergott DJ, Frank SA, Roush WR. Proc Natl Acad Sci U S A 2004;101:11955. [PubMed: 15173590]For use of PyBroP: Coste J, Frerot E, Jouin P. J Org Chem 1994;59:2437.For use of DCC: Xia Z, Smith CD. J Org Chem 2001;66:3459. [PubMed: 11348130]

36. (a) Otera J, Danoh N, Nozaki H. J Org Chem 1991;56:5307. (b) Orita A, Sakamoto K, Hamada Y, Mitsutome A, Otera J. Tetrahedron 1999;55:2899. (c) Orita A, Mitsutome A, Otera J. J Org Chem 1998;63:2420. [PubMed: 11672094]

37. (a) Kita Y, Maeda H, Omori K, Okuno T, Tamura Y. Synlett 1993:273. (b) Kita Y, Maeda H, Omori K, Okuno T, Tamura Y. J Chem Soc Perkin Trans 1993;1:2999. (c) Trost BM, Chisholm JD. Org Lett 2002;4:3743. [PubMed: 12375933]

38. For reviews of enolate alkylations: (a)EvansDAMorrisonJDAsymmetric SynthesisAcademic PressNew York198431(b)CaineDPattendenGComprehensive Organic SynthesisPergamon PressOxford199131

39. Takacs JM, Jaber MR, Swanson BJ, Mehrman SJ. Tetrahedron: Asymmetry 1998;9:4313.

40. Acid 9 has been previously synthesized in racemic form: (a) Donaldson WA, Craig R, Spanton S. Tetrahedron Letters 1992;33:3967. (b) Wasicak JT, Craig RA, Henry R, Dasgupta B, Li H, Donaldson WA. Tetrahedron 1997;53:4185.

41. For reviews on olefin metathesis: (a) Gradillas A, Perez-Castells J. Angew Chem Int Ed 2006;45:6086. (b) Nicolaou KC, Bulger PG, Sarlah D. Angew Chem, Int Ed 2005;44:4490. (c) Fürstner A. Angew Chem, Int Ed 2000;39:3012.

42. For recent examples of diene or triene forming ring closing metatheses: (a) Biswas K, Lin H, Njardarson JT, Chappell MD, Chou TC, Guan Y, Tong WP, He L, Horwitz SB, Danishefsky SJ. J Am Chem Soc 2002;124:9825. [PubMed: 12175242] (b) Evano G, Schaus JV, Panek JS. Org Lett 2004;6:525. [PubMed: 14961614] (c) Fürstner A, Nevado C, Tremblay M, Chevrier C, Teply F, Aissa C, Waser M. Angew Chem, Int Ed 2006;45:5837.

43. (a) Fürstner A, Dierkes T. Org Lett 2000;2:2463. [PubMed: 10956522] (b) Evans PA, Cui J, Gharpure SJ, Polosukhin A, Zhang H-R. J Am Chem Soc 2003;125:14702. [PubMed: 14640634]

44. Wang YG, Kobayashi Y. Org Lett 2002;4:4615. [PubMed: 12489943]

45. (a) Crimmins MT, She J. J Am Chem Soc 2004;126:12790. [PubMed: 15469270] (b) Trost BM, Frederiksen MU, Papillon JPN, Harrington PE, Shin S, Shireman BT. J Am Chem Soc 2005; 127:3666. [PubMed: 15771479]

46. Ono K, Nagata T, Nishida A. Synlett 2003:1207.

47. Sharma S, Oehlschlager AC. J Org Chem 1989;54:5064.

48. (a) De Riccardis F, Minale L, Riccio R, Giovannitti B, Iorizzi M, Debitus C. Gazz Chim Ital 1993;123:79. (b) Finamore E, Minale L, Riccio R, Rinaldo G, Zollo F. J Org Chem 1991;56:1146.

49. (a) Roush WR, Palkowitz AD, Ando K. J Am Chem Soc 1990;112:6348. (b) Kalesse M, Chary KP, Quitschalle M, Burzlaff A, Kasper C, Scheper T. Chem--Eur J 2003;9:1129.

50. Fuwa H, Okamura Y, Natsugari H. Tetrahedron 2004;60:5341.

51. Harada, N.; Nakanishi, K. Circular Dichroic Spectrometry: Exciton Coupling in Organic Stereochemistry. University Science Books; Mill Valley, CA: 1983.

52. Lo LC, Berova N, Nakanishi K, Schlingmann G, Carter GT, Borders DB. J Am Chem Soc 1992;114:7371.

53. Keck GE, Romer DR. J Org Chem 1993;58:6083.

54. Fürstner has postulated the formation of a ketene intermediate during an attempted Yamaguchi macrocyclization. This postulated ketene intermediate ultimately led to the formation of an undesired 
substituted phenol: Fürstner A, Aissa C, Chevrier C, Teply F, Nevado C, Tremblay M. Angew Chem, Int Ed 2006;45:5832.. Although it is possible that acid 13 (Table 2) could have suffered from a similar side-reaction pathway, the expect side product(s) (ortho-methylphenol or acylated orthomethylphenol) was not observed

55. (a) Hodous BL, Ruble JC, Fu GC. J Am Chem Soc 1999;121:2637. (b) Wiskur SL, Fu GC. J Am Chem Soc 2005;127:6176. [PubMed: 15853315]

56. Curran DP, Zhang Q, Lu H, Gudipati V. J Am Chem Soc 2006;128:9943. [PubMed: 16866554] 

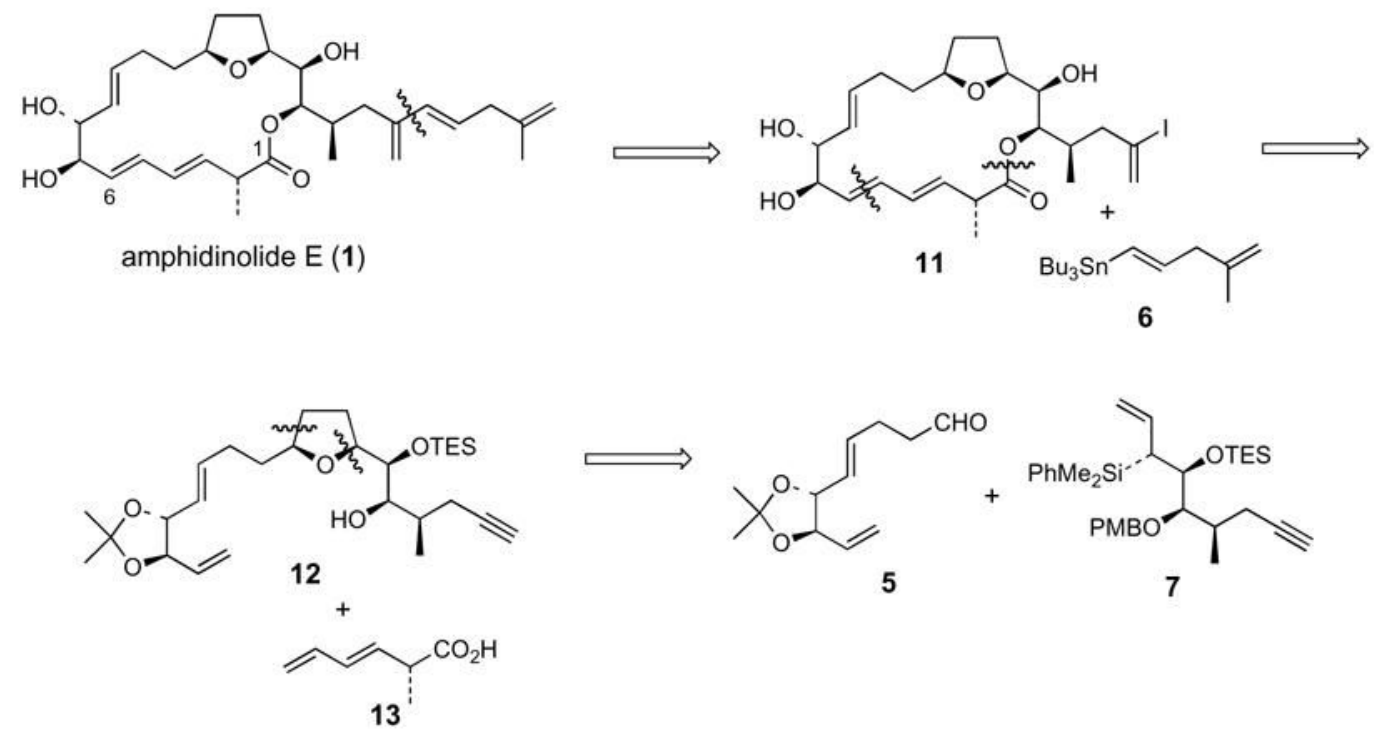

Figure 1. Retrosynthetic analysis of amphidinolide $\mathbf{E}$ 


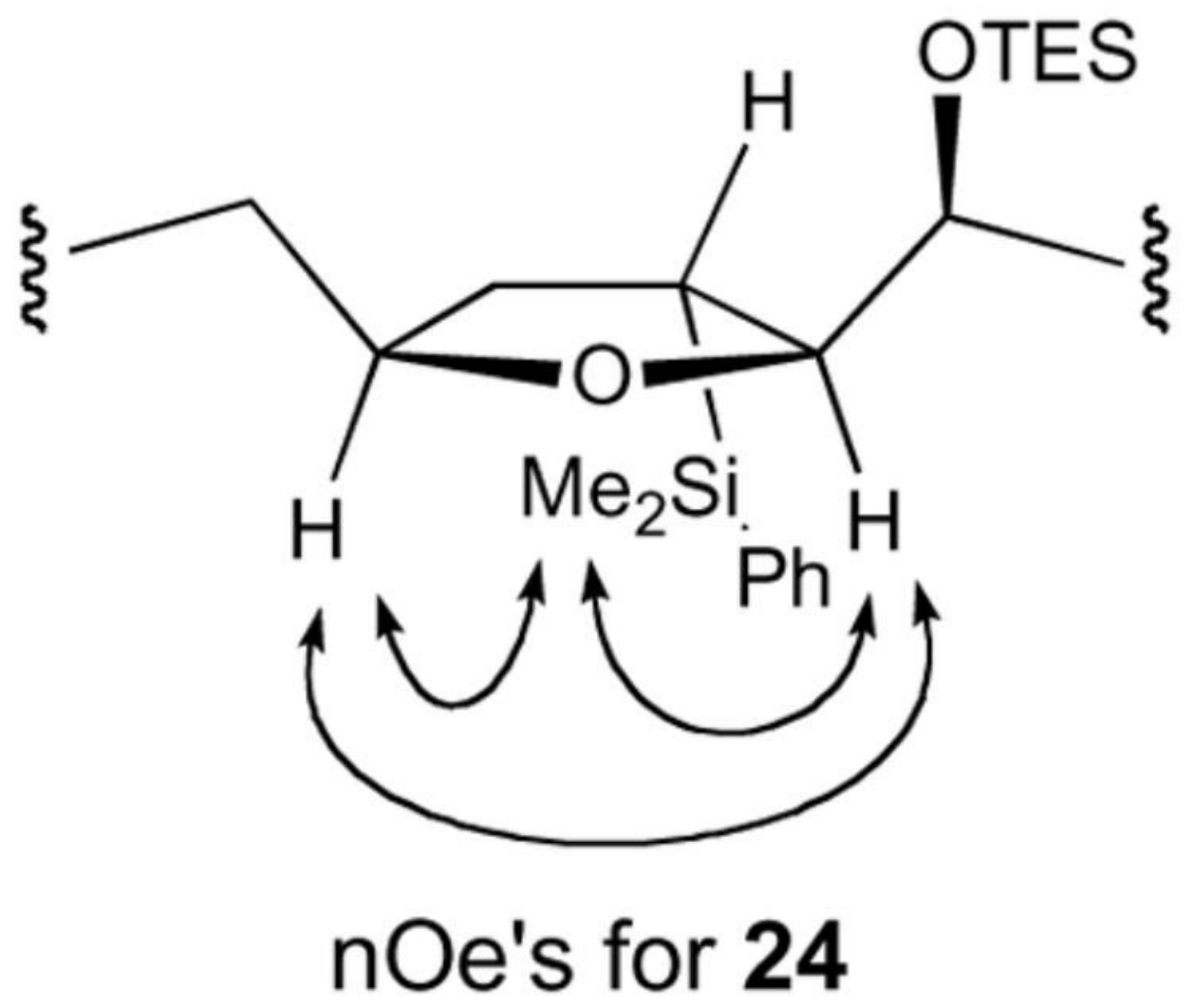

Figure 2. nOe analysis of 24 

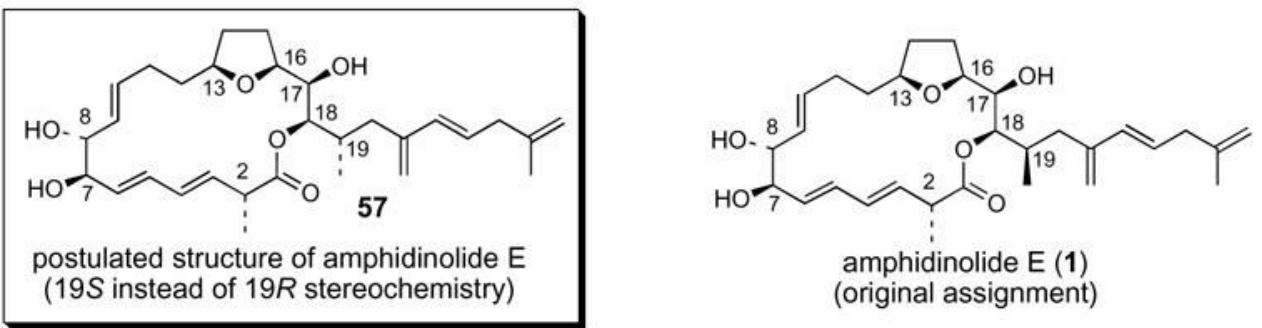

Figure 3. Postulated revised structure of amphidinolide E 


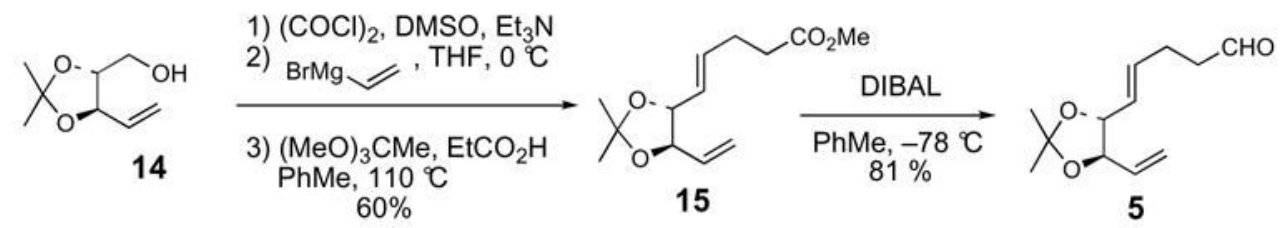

Scheme 1. Synthesis of aldehyde 5 

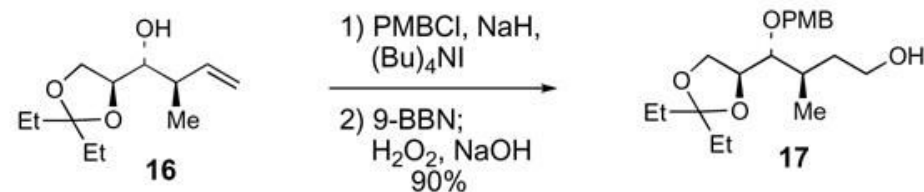

1) $\mathrm{SO}_{3} \cdot \mathrm{Pyr}$, DMSO, $\mathrm{EtN}(i-\mathrm{Pr})_{2}$

2) $\mathrm{PPh}_{3}, \mathrm{CBr}_{4}$; $n-B u L i,-78^{\circ} \mathrm{C}$ $88 \%$
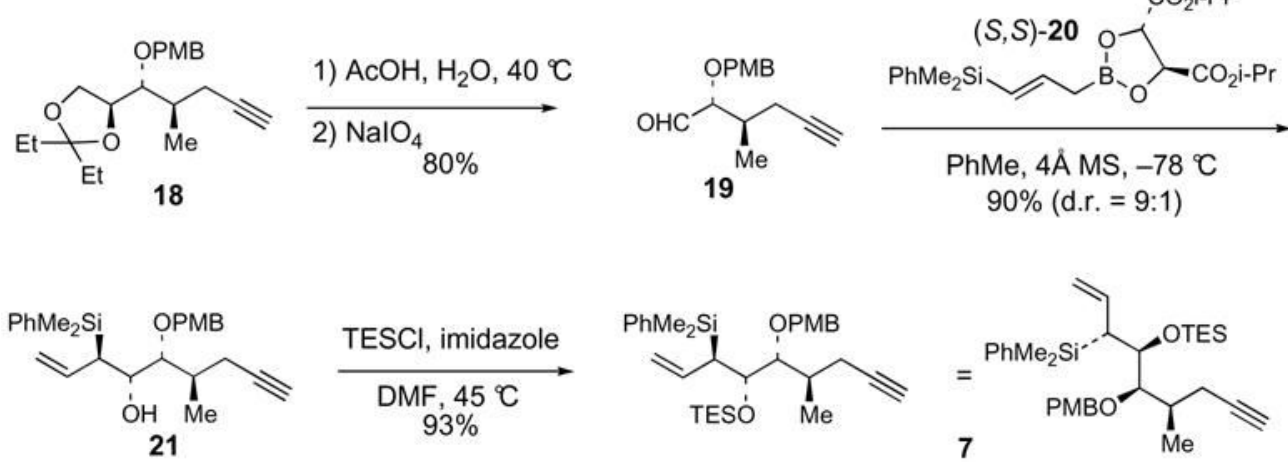

Scheme 2. Synthesis of allylsilane 7 

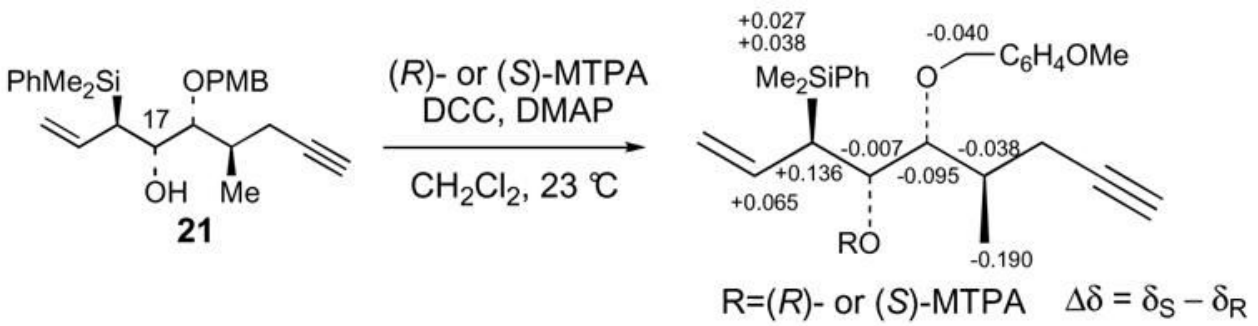

22
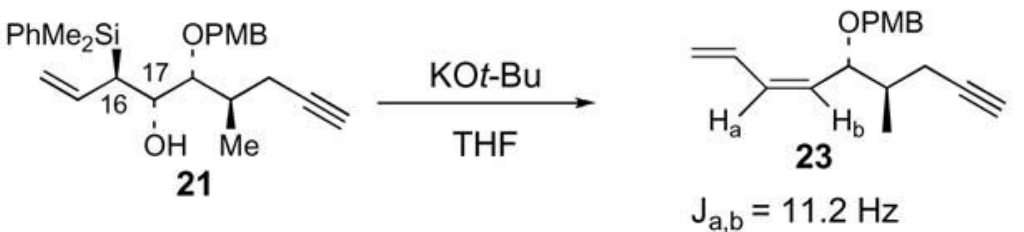

Scheme 3. Assignment of the C17 absolute and C16-C17 relative stereochemistry of allylsilane 21 


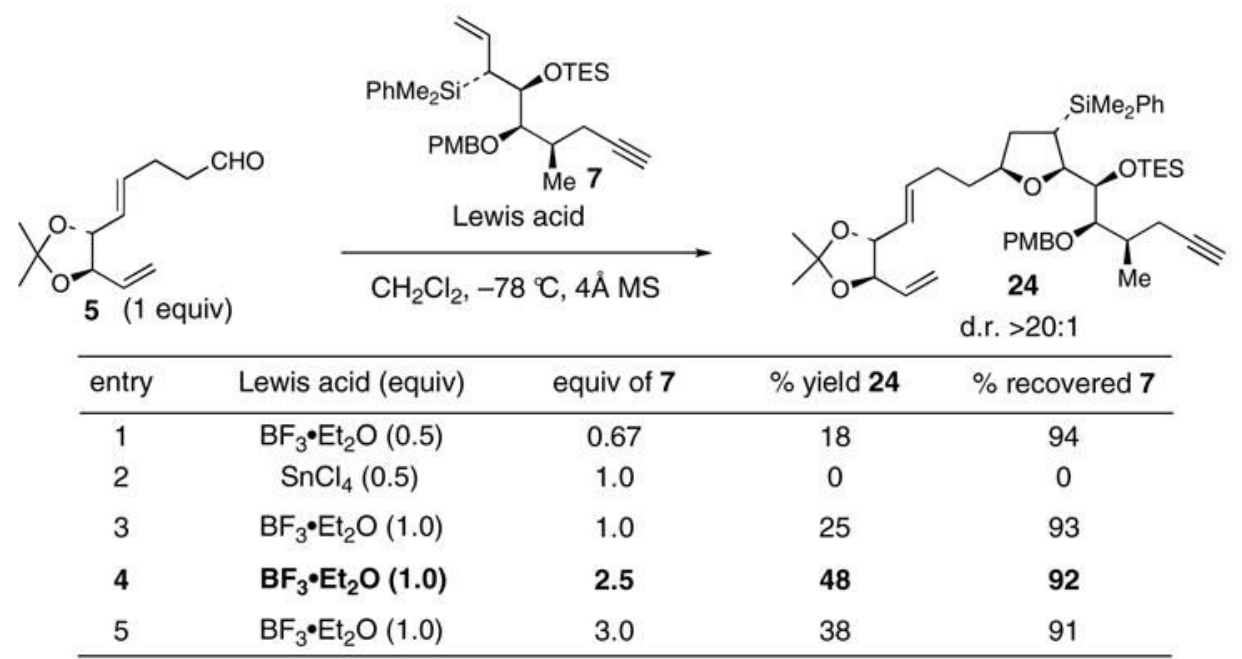

1) TBAF• $3 \mathrm{H}_{2} \mathrm{O}, \mathrm{DMF}, 90 \mathrm{C}$ 2) TESOTf, $\mathrm{Et}_{3} \mathrm{~N}$ 3) ${ }_{76 \%}$<smiles>C#CCC(C(O)C(O[Si])C1CCC(CC/C=C/C2OC(C)(C)OC2C=C)O1)[N+](=O)[O-]</smiles>

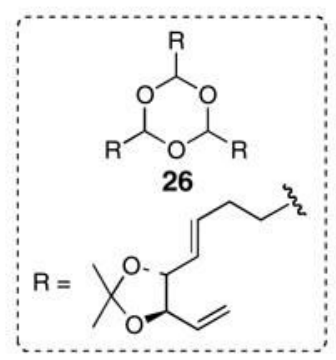

Scheme 4. [3+2] annulation of 5 and 7 

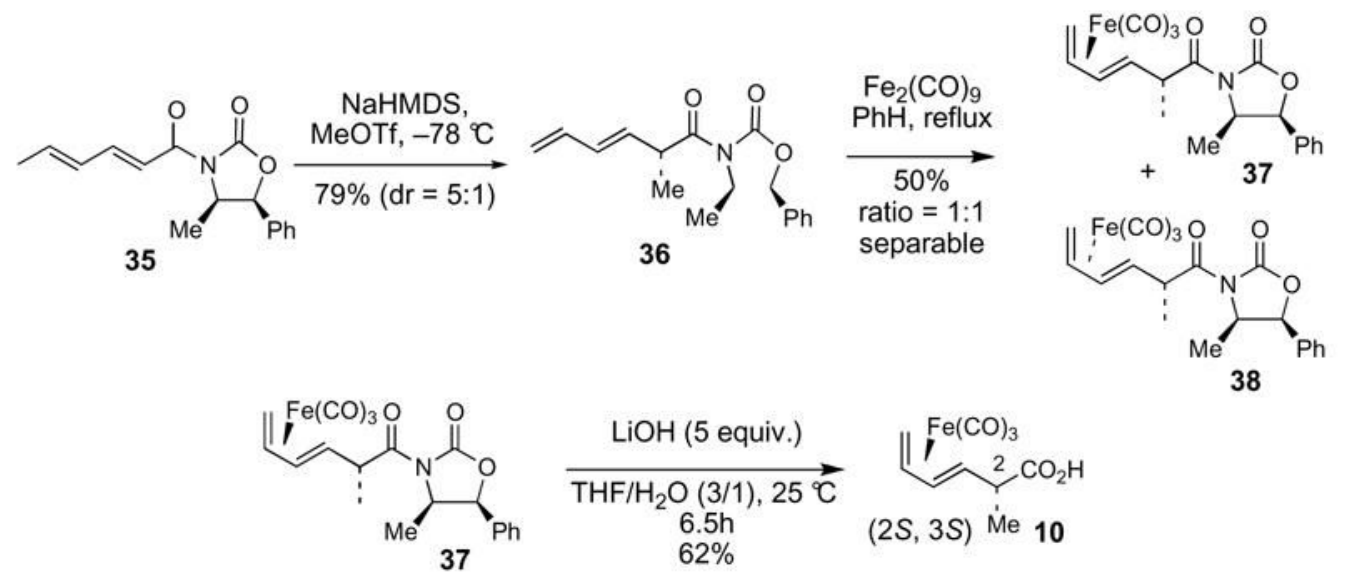

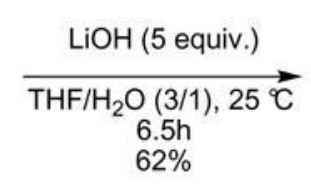<smiles>C=CC=CC(C(=O)O)C(=O)OC(=O)C(=O)O</smiles><smiles>CC(/C=C\c1ccccc1)C(=O)N1C(=O)OC(c2ccccc2)C1C(=O)OC(=O)OCc1ccccc1</smiles>

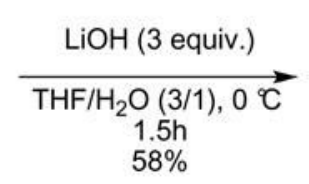

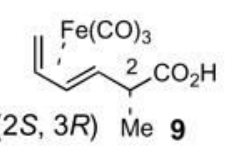

Scheme 5. Synthesis of acids 9 and 10 


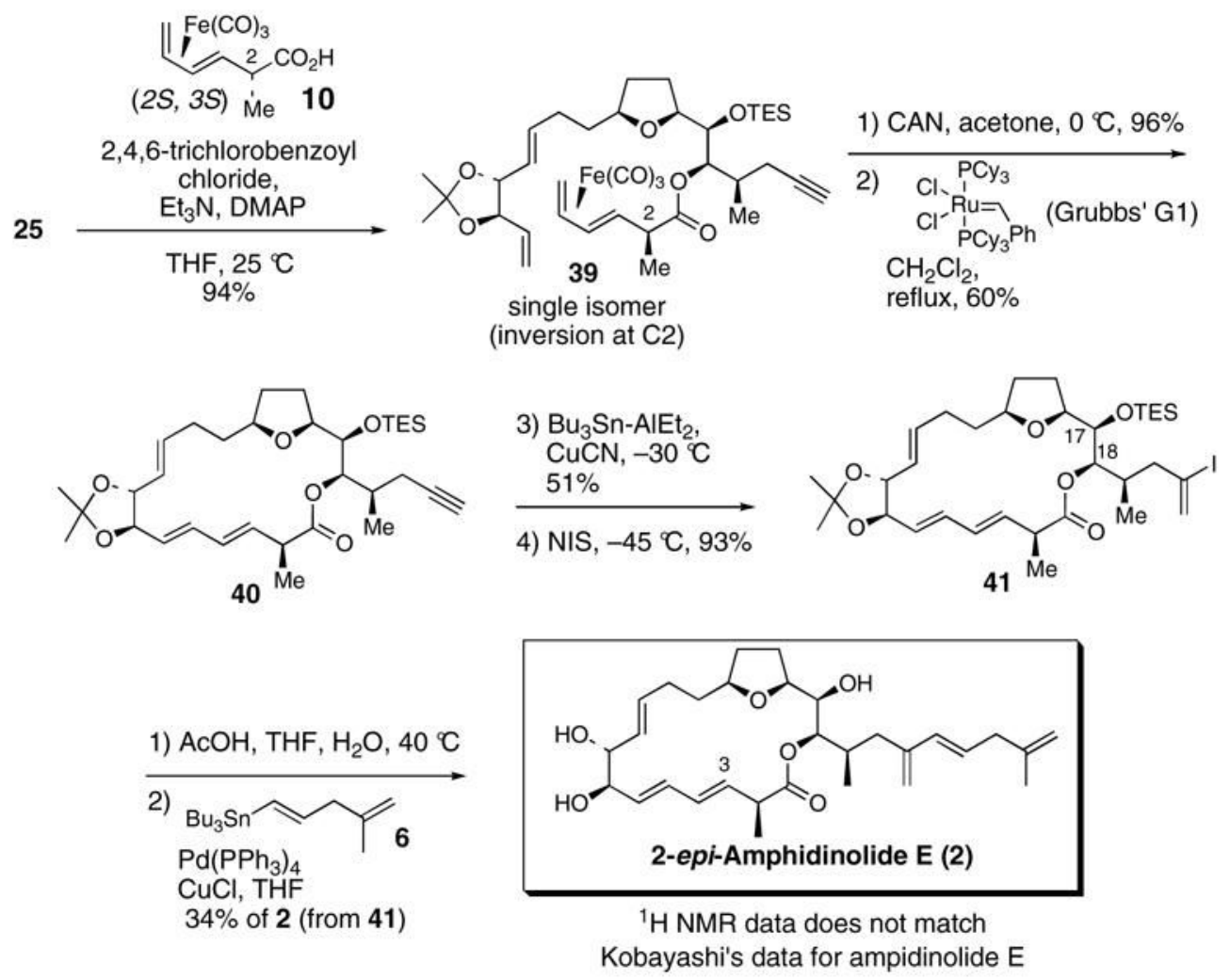

Scheme 6. Completion of 2-epi-amphidinolide E synthesis 


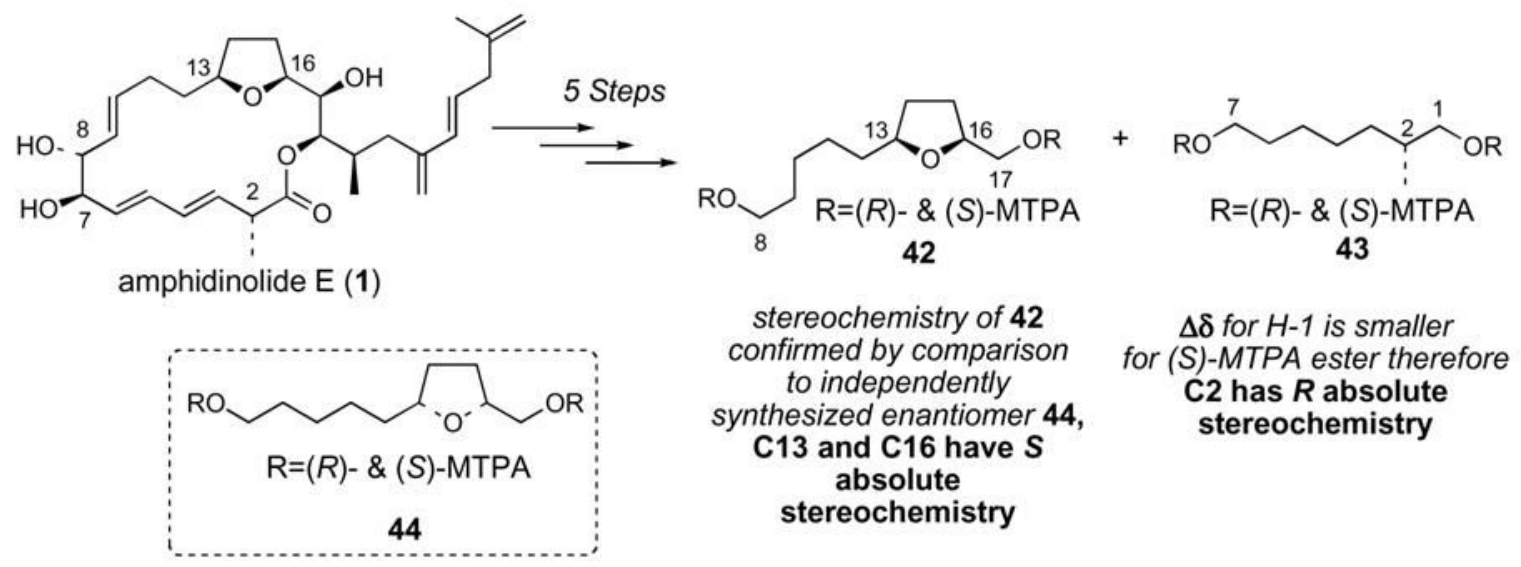

Scheme 7. Kobayashi's absolute stereochemical assignment of $13 S, 16 S$, and $2 R$ 


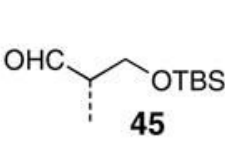

45

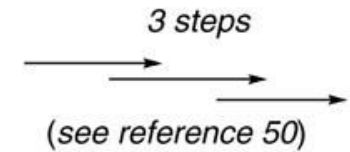

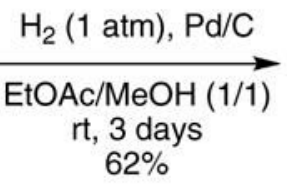

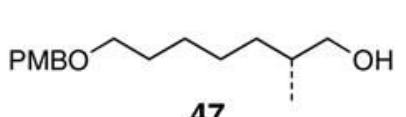

47
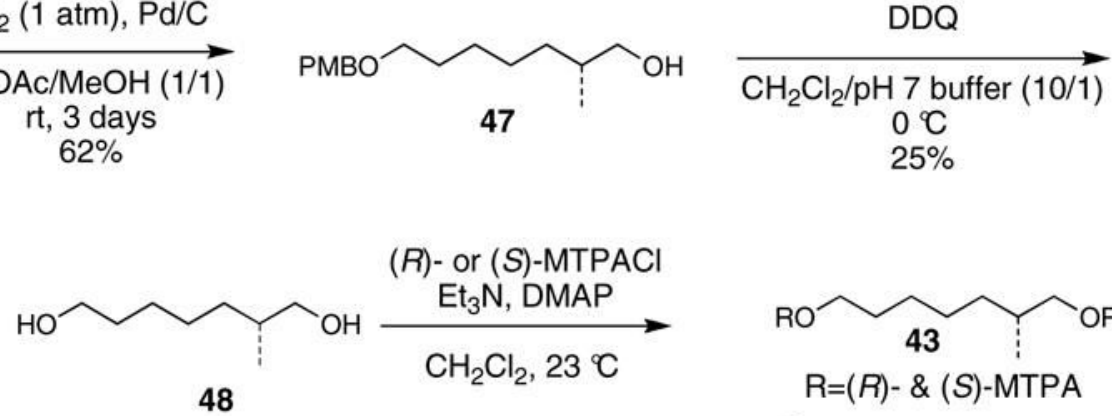

Scheme 8. Synthetic confirmation of $2 R$ stereochemistry of 43

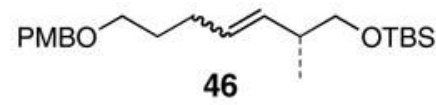

$25 \%$

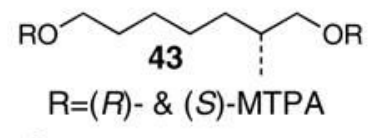

${ }^{1} \mathrm{H}$ NMR data matches

Kobayashi's data 


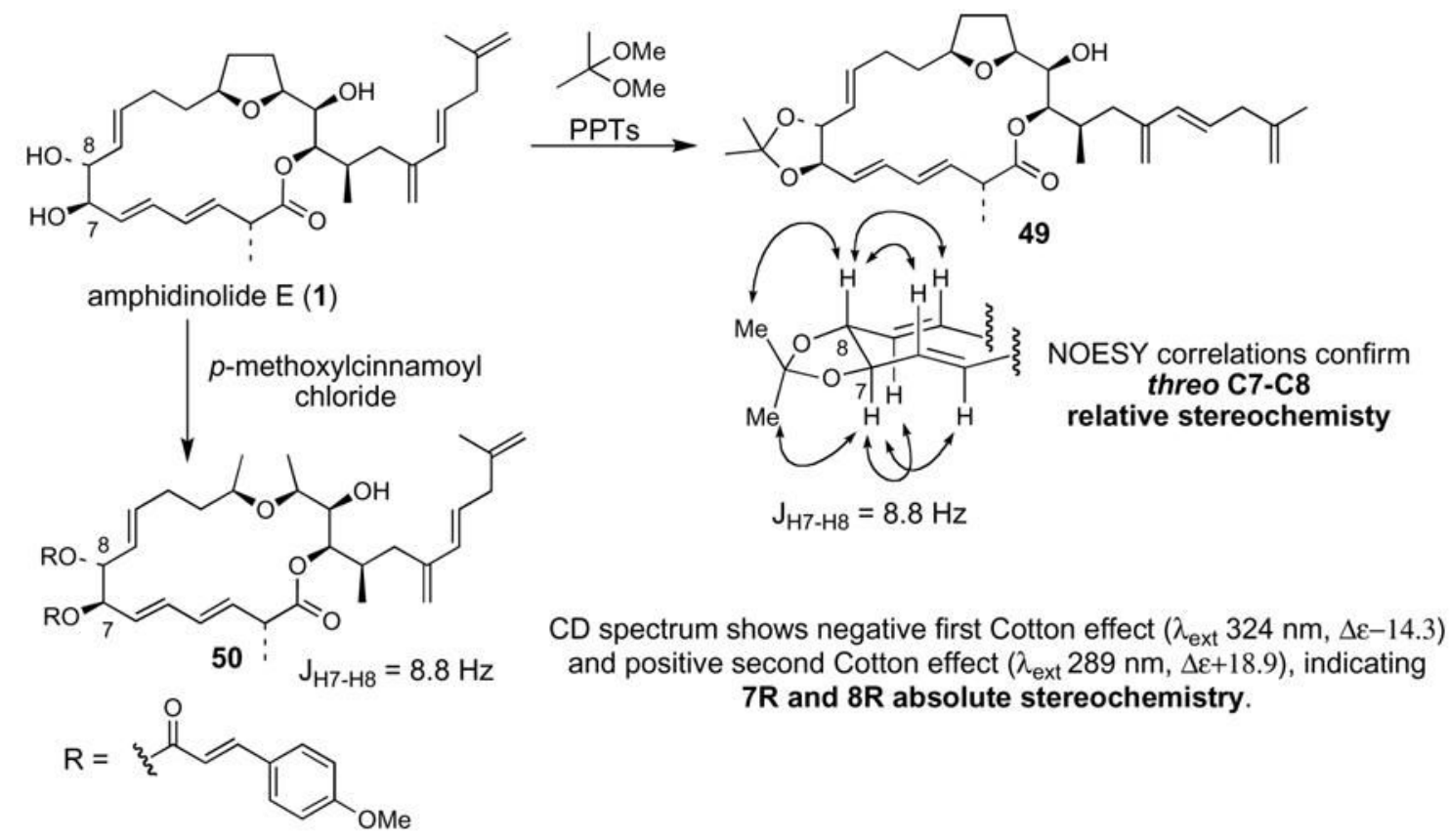

Scheme 9. Kobayashi's relative and absolute stereochemical assignment of C7 and C8 
a)

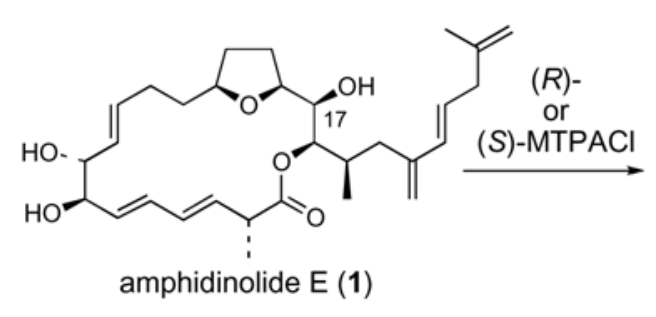

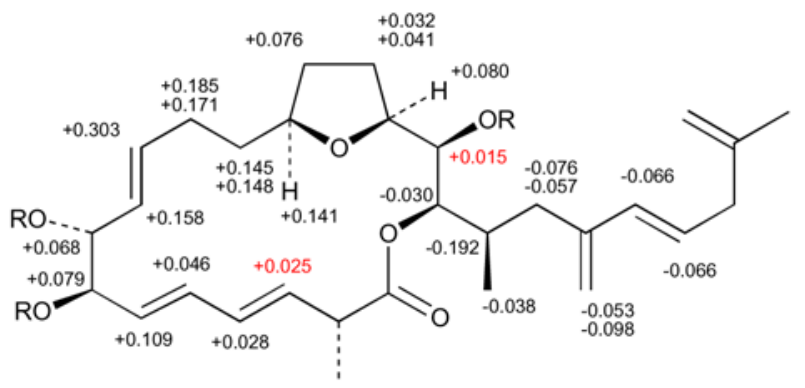

Kobayashi's Mosher Triester 51
$\mathrm{R}=(R)-\&(S)-\mathrm{MTPA}$ $\Delta \delta=\delta_{\mathrm{S}}-\delta_{\mathrm{R}}$

b)

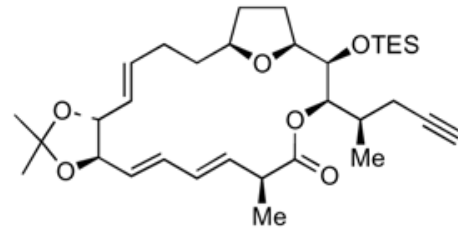

40

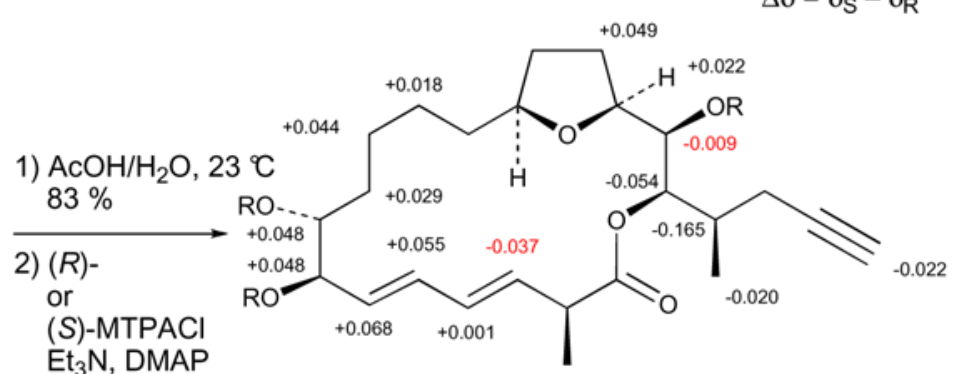
$\mathrm{CH}_{2} \mathrm{Cl}_{2}, 23^{\circ} \mathrm{C}$
Synthetic Mosher Triester

52

Scheme 10.

a) Kobayashi's absolute stereochemical assignment of C17 (also confirming C7 and C8). b) Comparison of Mosher ester data for synthetic $\mathbf{5 2}$ and natural product derivative $\mathbf{5 1}$. 


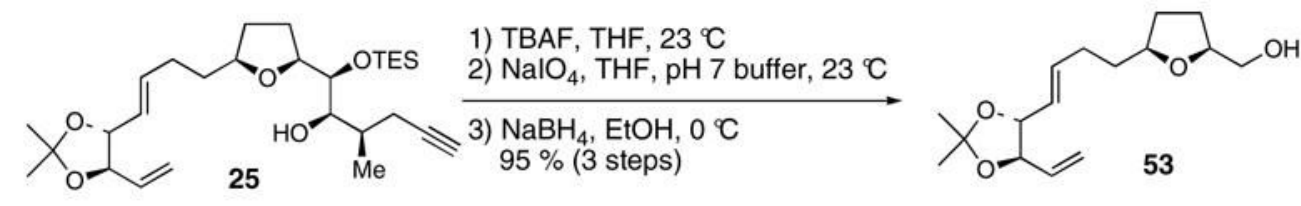

1) $\mathrm{H}_{2}, \mathrm{Pd} / \mathrm{C}, \mathrm{EtOAc}, \mathrm{MeOH}$

2) $\mathrm{AcOH} / \mathrm{H}_{2} \mathrm{O}(4 / 1), 40^{\circ} \mathrm{C}$

3) $\mathrm{NaIO}_{4}, \mathrm{THF}, \mathrm{pH} 7$ buffer, $23^{\circ} \mathrm{C}$

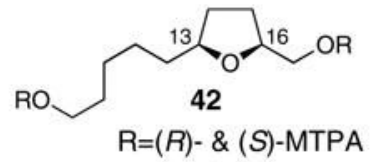

${ }^{1} \mathrm{H}$ NMR data matches Kobayashi's data
1) $\mathrm{NaBH}_{4}, \mathrm{EtOH}, \mathrm{O}^{\circ} \mathrm{C}$

2) $(R)$ - or (S)-MTPACl $\mathrm{Et}_{3} \mathrm{~N}, \mathrm{DMAP}, \mathrm{CH}_{2} \mathrm{Cl}_{2}, 23^{\circ} \mathrm{C}$

$36 \%$ (5 steps)

Scheme 11. Confirmation of $13 S$ and $16 S$ stereochemistry of 25 


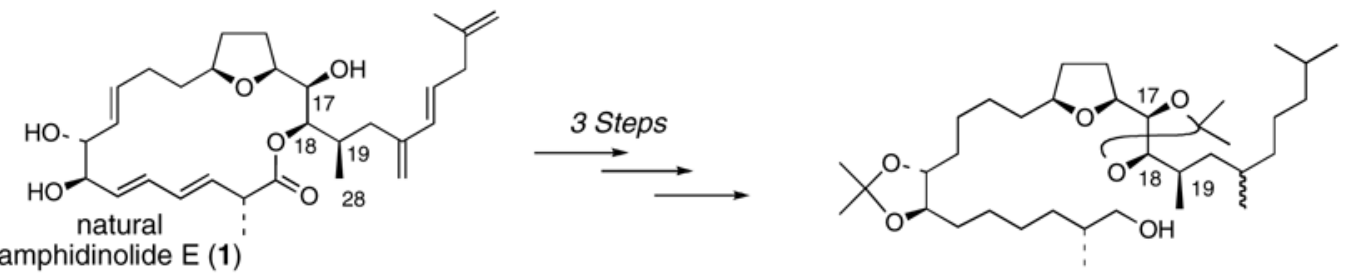

$\mathrm{J}_{\mathrm{H} 18-\mathrm{H} 19}=9.7 \mathrm{~Hz}, \mathrm{~J}_{\mathrm{H} 18-\mathrm{C} 28}=3.0 \mathrm{~Hz}$ supports erythro $\mathrm{C} 18-\mathrm{C} 19$
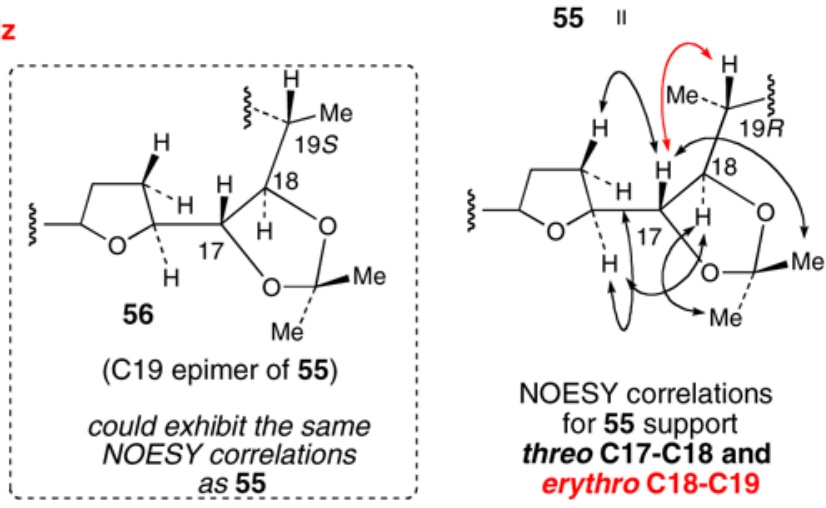

NOESY correlations for $\mathbf{5 5}$ support threo $\mathrm{C} 17-\mathrm{C} 18$ and erythro $\mathrm{C} 18-\mathrm{C} 19$

Scheme 12. Kobayashi's C17-C18 and C18-C19 relative stereochemical assignment 


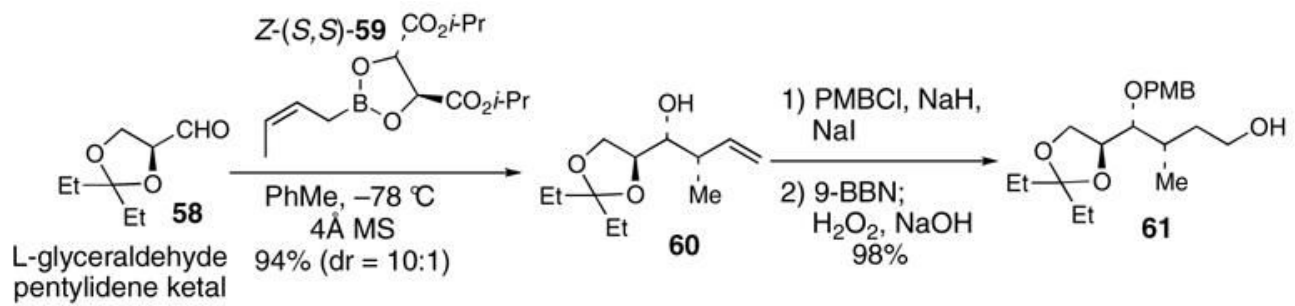

1) $\mathrm{SO}_{3} \cdot \mathrm{Pyr}, \mathrm{DMSO}$ $\mathrm{EtN}(i-\mathrm{Pr})_{2}$
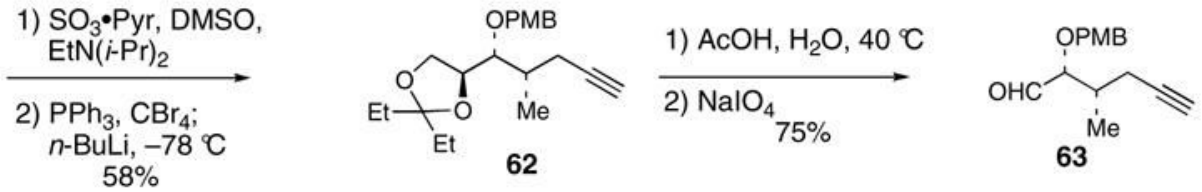

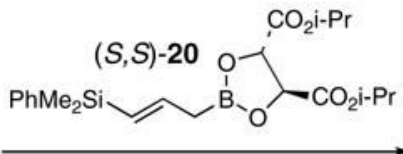

$\mathrm{PhMe}, 4 \AA \AA \mathrm{MS},-78^{\circ} \mathrm{C}$ $90 \%$ (d.r. $=10: 1)$

Scheme 13. Synthesis of 19-epi-allylsilane 8 


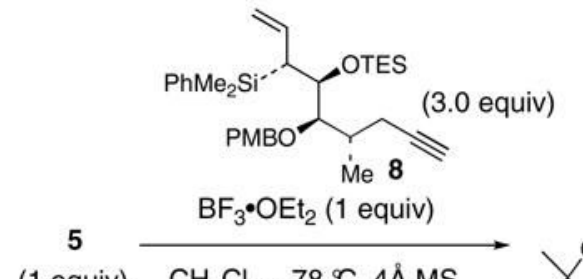

(1 equiv)

$\mathrm{CH}_{2} \mathrm{Cl}_{2},-78^{\circ} \mathrm{C}, 4 \AA \mathrm{AS}$ $61 \%$ (d.r. >20:1)

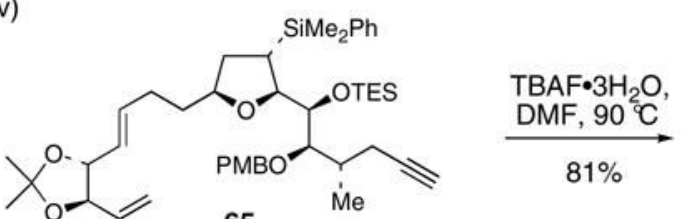
and

65

$92 \%$ of recovered 8
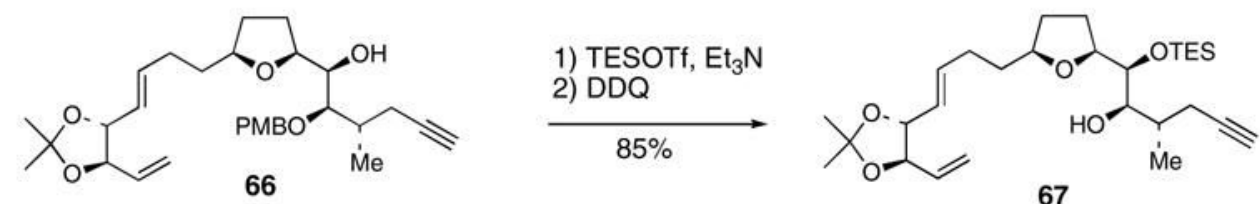

Scheme 14. [3+2] Annulation of 5 and 8 

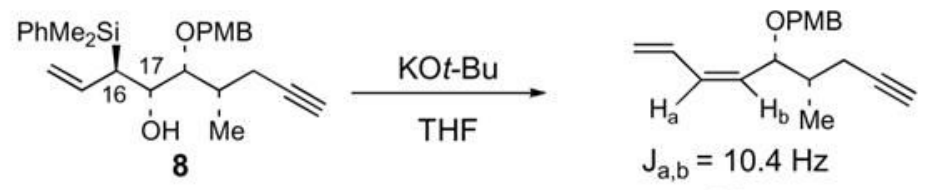

68
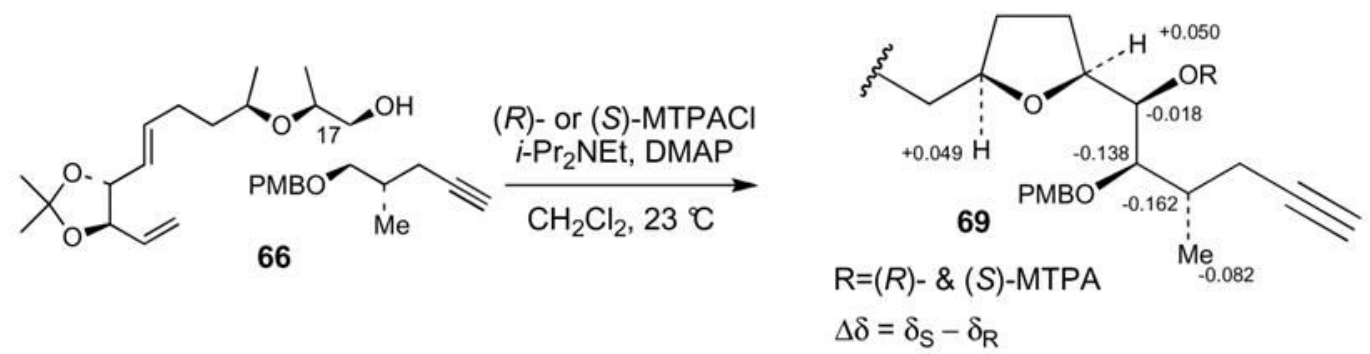

Scheme 15. Confirmation of the $\mathrm{C} 16-\mathrm{C} 17$ relative and $\mathrm{C} 17$ absolute stereochemistry 

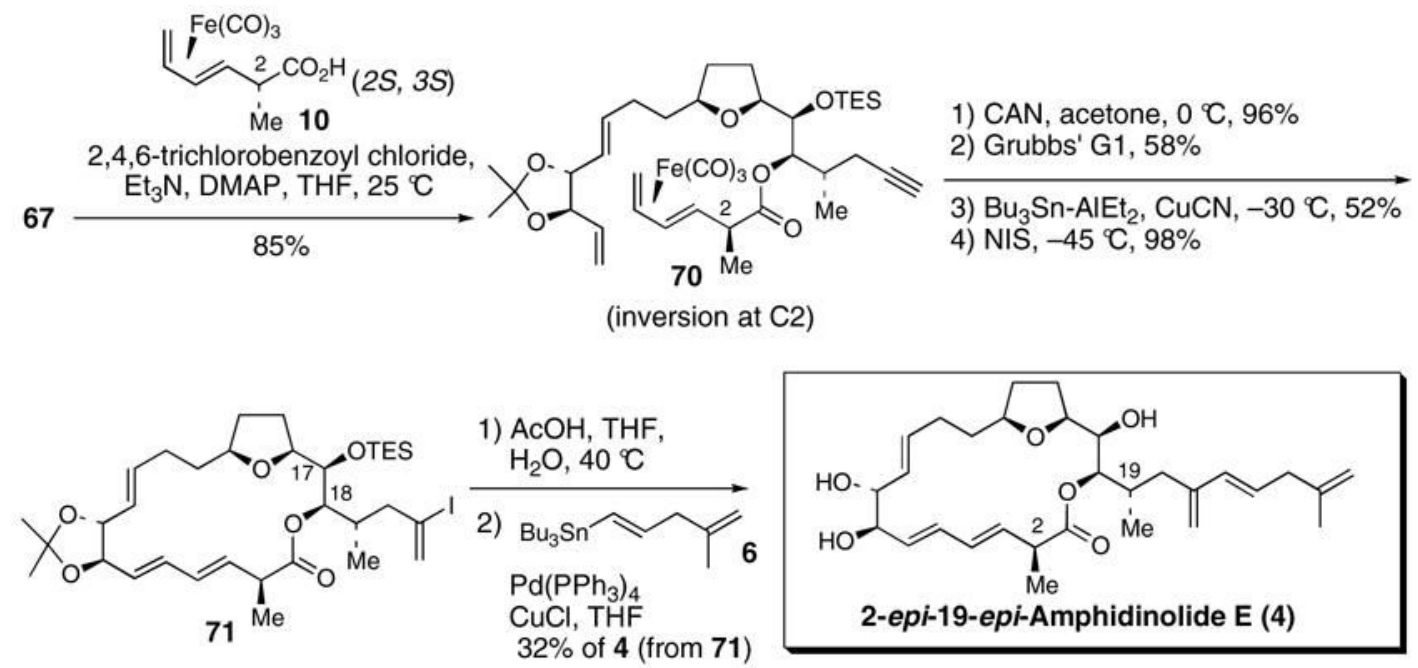

$\left({ }^{1} \mathrm{H}\right.$ NMR data does not match Kobayashi's data for ampidinolide E)

Scheme 16. Completion of 2-epi-19-epi-amphidinolide E synthesis 


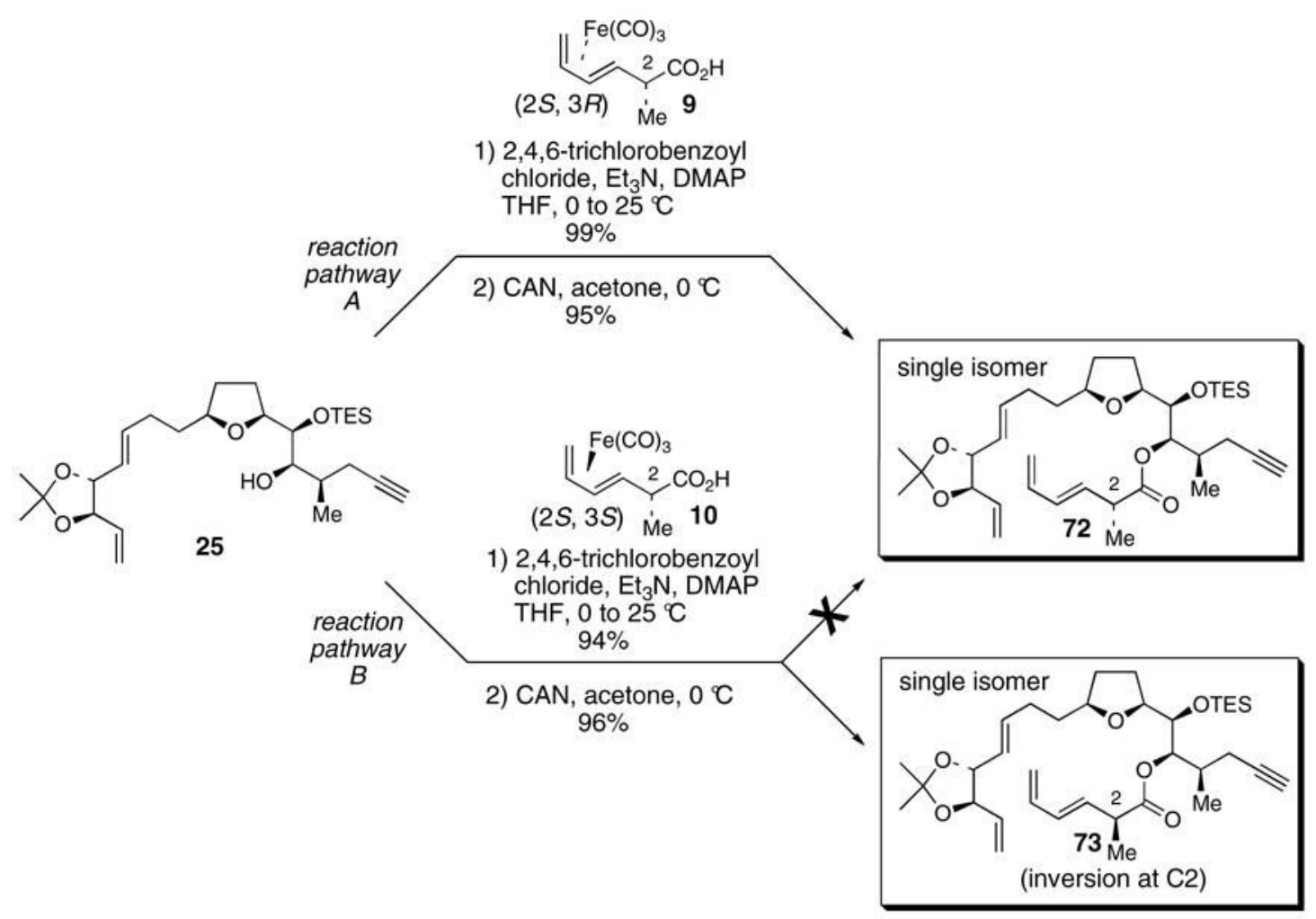

Scheme 17. Divergent behavior of acids 9 and 10 in the modified Yamaguchi esterification reaction of 25 

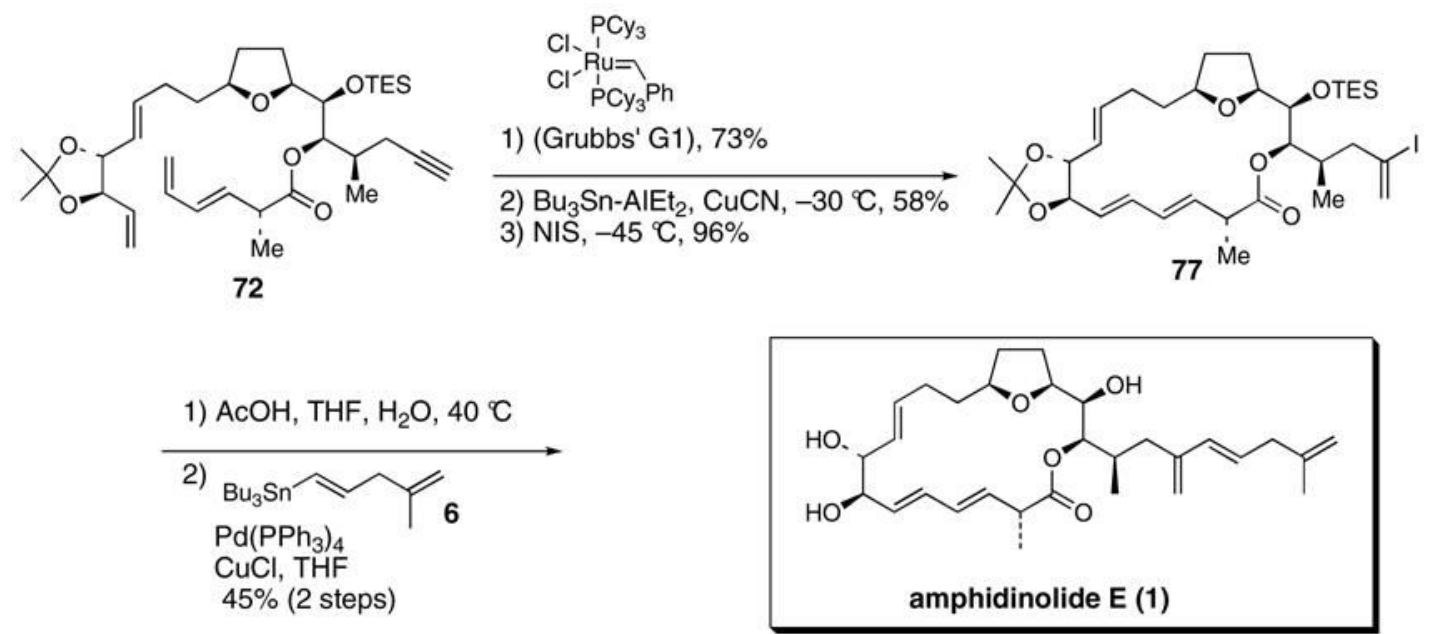

${ }^{1} \mathrm{H}$ NMR data matches

Kobayashi's data for ampidinolide $\mathrm{E}$

Scheme 18. Completion of the synthesis of amphidinolide $\mathbf{E}$ 


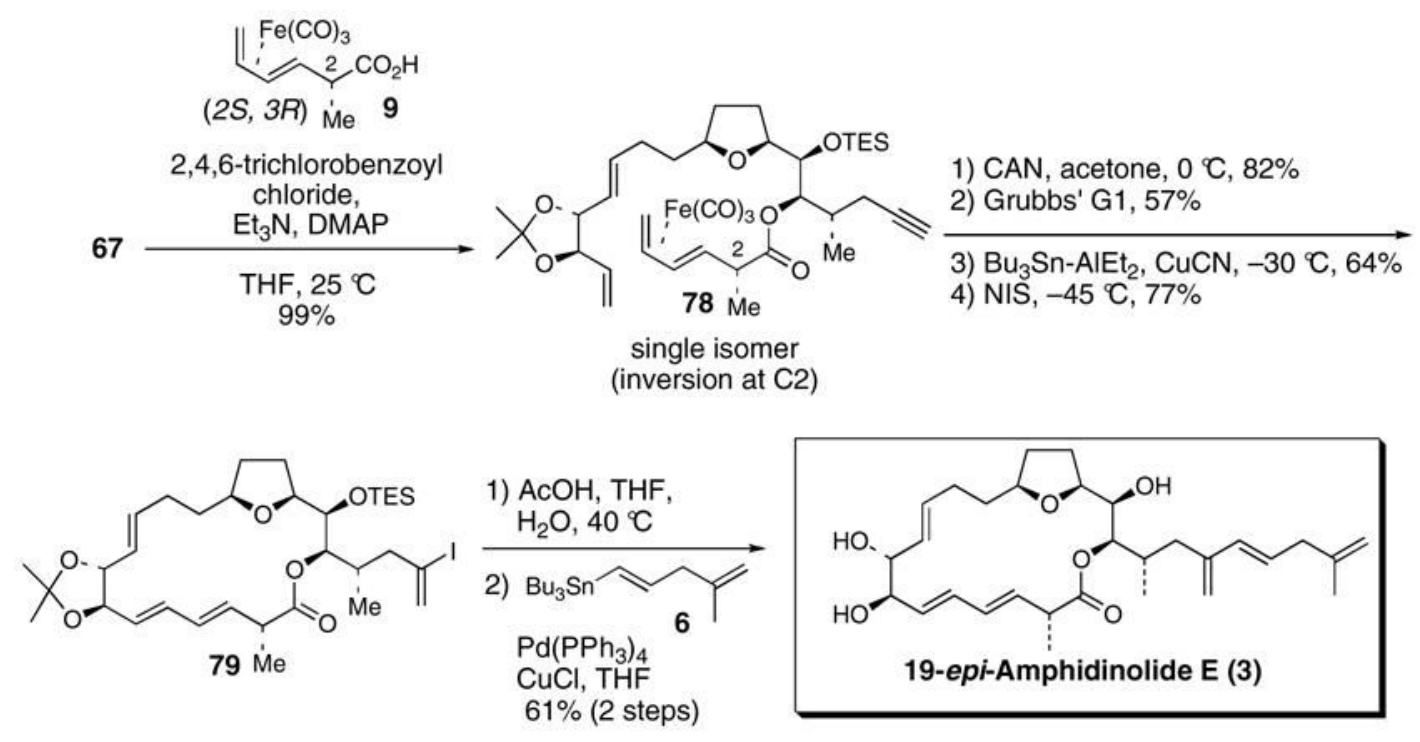

Scheme 19. Completion of the synthesis of 19-epi-amphidinolide E 
<smiles>C=CC(C=C(C(=O)O)C(=O)O)C(=O)O</smiles>

10
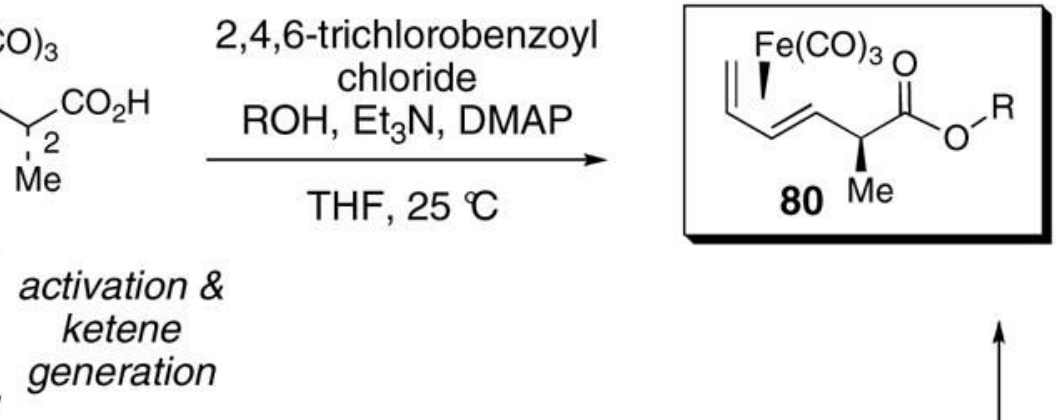

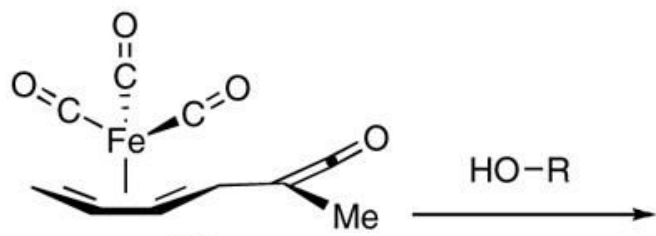

81

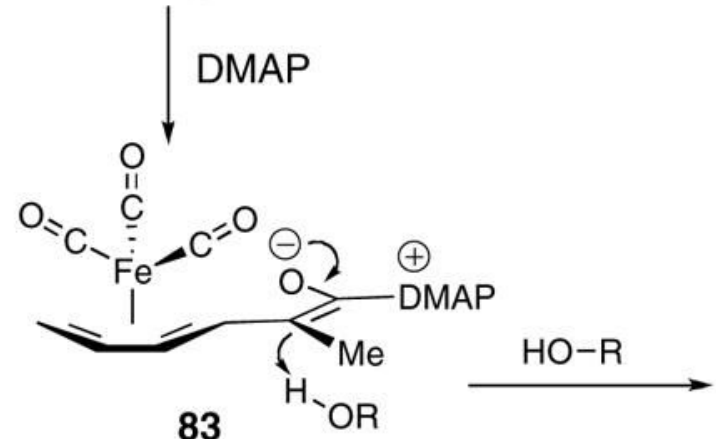

83
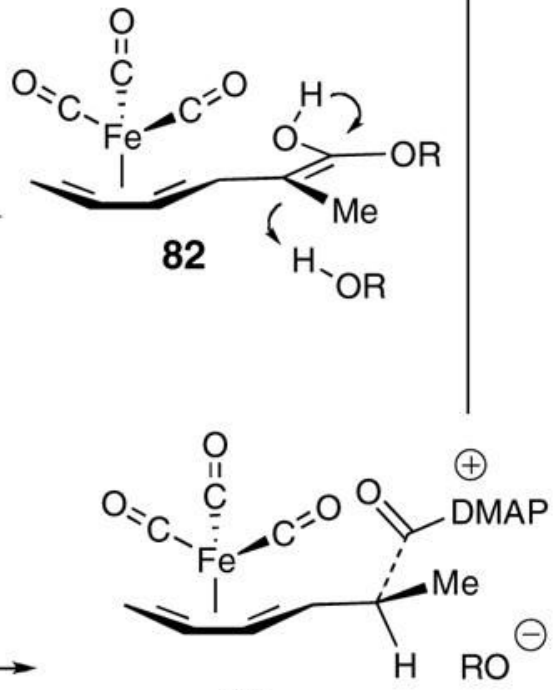

84 
Table 2

Assessment of C2 stereochemistry of 36, 9, 10, 76, and 39

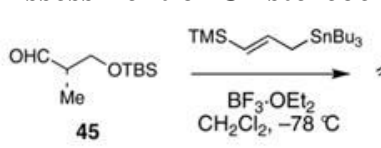<smiles>C=CC(O)C(O)C(C)COC</smiles>
74

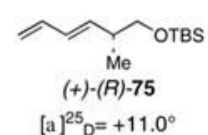

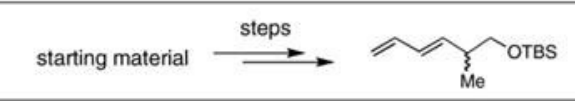

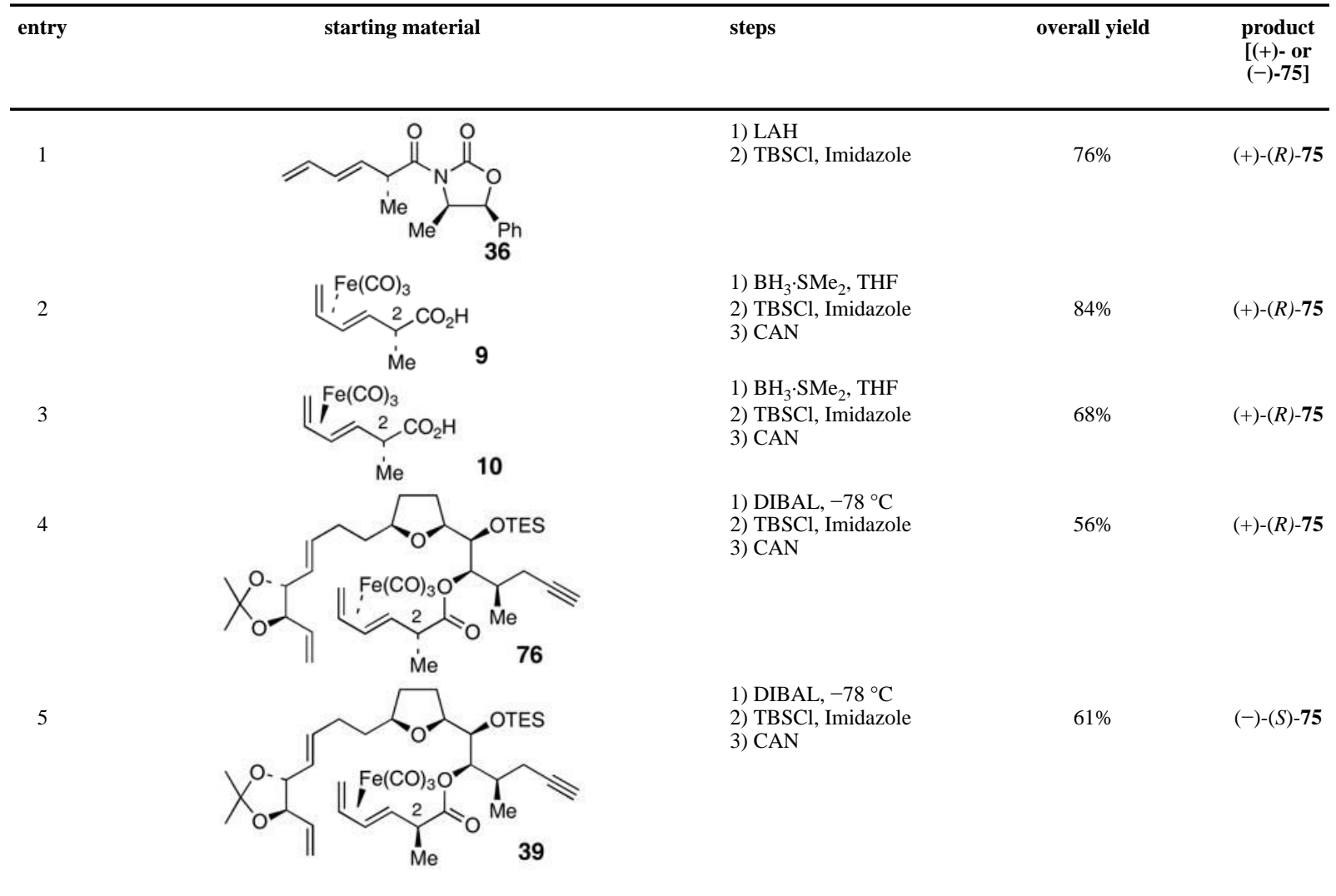


Table 3

Spectroscopic comparison of natural and synthetic amphidinolide E (1) ${ }^{1} \mathrm{H}$ NMR Data<smiles>[13CH3]CC([14CH3])C[18OH]</smiles>

45<smiles>CCCCCCCCCCCCCCCCCC</smiles>

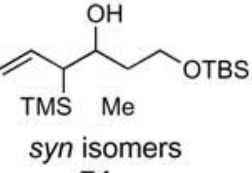

74

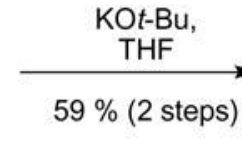

$59 \%$ (2 steps)<smiles>[M]C(/C=C/C=C)CO[Sb]</smiles>

$(+)-(R)-75$

$[\alpha]^{25}=+11.0^{\circ}$ 
Table 4

Partial spectroscopic details of the four amphidinolide E stereoisomers 1-4

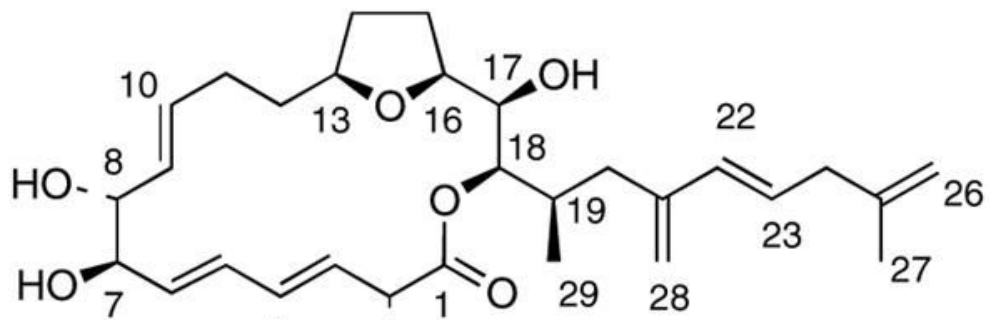

30

\begin{tabular}{|c|c|c|c|c|}
\hline $\mathbf{C}(\mathbf{H})$ & $\begin{array}{l}\text { Synthetic Amphidinolide } \\
\text { E (1) }\end{array}$ & 2-epi-Amphidinolide E (2) & 19-epi-Amphidinolide E (3) & $\begin{array}{l}\text { 2-epi-19-epi- } \\
\text { Amphidinolide E (4) }\end{array}$ \\
\hline 2 & $3.26(1 \mathrm{H}, \mathrm{m})$ & $3.35(1 \mathrm{H}, \mathrm{q}, \mathrm{J}=5.2)$ & $3.26(1 \mathrm{H}, \mathrm{m})$ & $3.34(1 \mathrm{H}, \mathrm{m})$ \\
\hline 3 & $\begin{array}{l}5.67(3 \mathrm{H}, \mathrm{m}) 3,10, \& 23 \\
\text { overlap }\end{array}$ & $6.00(1 \mathrm{H}, \mathrm{m})$ & $\begin{array}{l}5.60(3 \mathrm{H}, \mathrm{m}) 3,6, \& 10 \\
\text { overlap }\end{array}$ & $5.98(1 \mathrm{H}, \mathrm{m})$ \\
\hline 4 & $6.19(2 \mathrm{H}, \mathrm{m}) 4 \& 5$ overlap & $6.20(2, \mathrm{~m}) 4 \& 5$ overlap & $6.19(2, \mathrm{~m}) 4$ \& 5 overlap & $6.20(2, \mathrm{~m}) 4$ \& 5 overlap \\
\hline 5 & $6.19(2 \mathrm{H}, \mathrm{m})$ & $6.20(2 . \mathrm{m})$ & $6.19(2 . \mathrm{m})$ & $6.20(2, \mathrm{~m})$ \\
\hline & $4 \& 5$ overlap & $4 \& 5$ overlap & $4 \& 5$ overlap & $4 \& 5$ overlap \\
\hline 6 & $5.53(1 \mathrm{H}, \mathrm{dd}, \mathrm{J}=14.4,8.8)$ & $\begin{array}{l}6.00(3 \mathrm{H}, \mathrm{m}) 6,10, \& 23 \\
\text { overlap }\end{array}$ & $\begin{array}{l}5.60(3 \mathrm{H}, \mathrm{m}) 3,6, \& 10 \\
\text { overlap }\end{array}$ & $\begin{array}{l}5.63(2 \mathrm{H}, \mathrm{m}) 6 \& 10 \\
\text { overlap }\end{array}$ \\
\hline 7 & $3.89(1 \mathrm{H}, \mathrm{t}, \mathrm{J}=8.8)$ & $\begin{array}{l}3.95(2 \mathrm{H}, \text { app dt, } \mathrm{J}=8.4,19.2) \\
7 \& 8 \text { overlap }\end{array}$ & $3.88(1 \mathrm{H}, \mathrm{t}, \mathrm{J}=8.8)$ & $3.96(2 \mathrm{H}, \mathrm{m}) 7 \& 8$ overlap \\
\hline 8 & $3.95(1 \mathrm{H}, \mathrm{t}, \mathrm{J}=8.4)$ & $\begin{array}{l}3.95(2 \mathrm{H}, \text { app } \mathrm{dt}, \mathrm{J}=8.4,19.2) \\
7 \text { \& } 8 \text { overlap }\end{array}$ & $3.95(1 \mathrm{H}, \mathrm{t}, \mathrm{J}=8.4)$ & $3.96(2 \mathrm{H}, \mathrm{m}) 7 \& 8$ overlap \\
\hline 9 & $5.27(1 \mathrm{H}, \mathrm{dd}, \mathrm{J}=14.4,7.6)$ & $5.27(1 \mathrm{H}, \mathrm{dd}, \mathrm{J}=7.6,14.4)$ & $5.27(1 \mathrm{H}, \mathrm{dd}, \mathrm{J}=8.0,15.2)$ & $5.30(1 \mathrm{H}, \mathrm{dd}, \mathrm{J}=7.6,15.2)$ \\
\hline 10 & $\begin{array}{l}5.67(3 \mathrm{H}, \mathrm{m}) 3,10, \& 23 \\
\text { overlap }\end{array}$ & $\begin{array}{l}6.00(3 \mathrm{H}, \mathrm{m}) 6,10, \& 23 \\
\text { overlap }\end{array}$ & $\begin{array}{l}5.60(3 \mathrm{H}, \mathrm{m}) 3,6, \& 10 \\
\text { overlap }\end{array}$ & $\begin{array}{l}5.63(2 \mathrm{H}, \mathrm{m}) 6 \& 10 \\
\text { overlap }\end{array}$ \\
\hline 13 & $3.40(1 \mathrm{H}, \mathrm{m})$ & $3.43(1 \mathrm{H}, \mathrm{m})$ & $3.40(1 \mathrm{H}, \mathrm{m})$ & $3.42(1 \mathrm{H}, \mathrm{m})$ \\
\hline 16 & $3.56(1 \mathrm{H}, \mathrm{m})$ & $3.50(1 \mathrm{H}, \mathrm{m})$ & $3.56(1 \mathrm{H}$, app q. $\mathrm{J}=8.8)$ & $3.50(1 \mathrm{H}, \mathrm{m})$ \\
\hline 17 & $3.72(1 \mathrm{H}, \mathrm{m})$ & $3.69(1 \mathrm{H}$, app t, J $=5.2)$ & $3.78(1 \mathrm{H}, \mathrm{d}, \mathrm{J}=7.2)$ & $3.74(1 \mathrm{H}, \mathrm{m})$ \\
\hline 18 & $4.66(1 \mathrm{H}, \mathrm{d}, \mathrm{J}=9.2)$ & $4.66(1 \mathrm{H}, \mathrm{d}, \mathrm{J}=9.6)$ & $4.68(1 \mathrm{H}, \mathrm{d}, \mathrm{J}=10.4)$ & $4.69(1 \mathrm{H}, \mathrm{d}, \mathrm{J}=10.4)$ \\
\hline 22 & $6.05(1 \mathrm{H}, \mathrm{d}, \mathrm{J}=15.2)$ & $6.04(1 \mathrm{H}, \mathrm{d}, \mathrm{J}=16.0)$ & $6.05(1 \mathrm{H}, \mathrm{d}, \mathrm{J}=15.6)$ & $6.05(1 \mathrm{H}, \mathrm{d}, \mathrm{J}=16.0)$ \\
\hline 23 & $\begin{array}{l}5.67(3 \mathrm{H}, \mathrm{m}) 3,10, \& 23 \\
\text { overlap }\end{array}$ & $\begin{array}{l}6.00(3 \mathrm{H}, \mathrm{m}) 6,10, \& 23 \\
\text { overlap }\end{array}$ & $5.87(1 \mathrm{H}, \mathrm{dt}, \mathrm{J}=6.8,16.0)$ & $5.85(1 \mathrm{H}, \mathrm{dt}, \mathrm{J}=7.2,15.6)$ \\
\hline $26 \mathrm{a}$ & $4.75(1 \mathrm{H}, \mathrm{s})$ & $4.74(1 \mathrm{H}, \mathrm{s})$ & $4.73(1 \mathrm{H}, \mathrm{s})$ & $4.74(1 \mathrm{H}, \mathrm{s})$ \\
\hline $26 \mathrm{~b}$ & $4.71(1 \mathrm{H}, \mathrm{s})$ & $4.70(1 \mathrm{H}, \mathrm{s})$ & $4.70(1 \mathrm{H}, \mathrm{s})$ & $4.70(1 \mathrm{H}, \mathrm{s})$ \\
\hline 27 & $1.72(3 \mathrm{H}, \mathrm{s})$ & $1.72(3 \mathrm{H}, \mathrm{s})$ & $1.71(3 \mathrm{H}, \mathrm{s})$ & $1.72(3 \mathrm{H}, \mathrm{s})$ \\
\hline $28 \mathrm{a}$ & $4.98(1 \mathrm{H}, \mathrm{s})$ & $4.98(1 \mathrm{H}, \mathrm{s})$ & $4.98(1 \mathrm{H}, \mathrm{s})$ & $4.98(1 \mathrm{H}, \mathrm{s})$ \\
\hline $28 \mathrm{~b}$ & $4.87(1 \mathrm{H}, \mathrm{s})$ & $4.86(1 \mathrm{H}, \mathrm{s})$ & $4.88(1 \mathrm{H}, \mathrm{s})$ & $4.88(1 \mathrm{H}, \mathrm{s})$ \\
\hline 29 & $0.92(3 \mathrm{H}, \mathrm{d}, \mathrm{J}=6.8)$ & $0.91(3 \mathrm{H}, \mathrm{d}, \mathrm{J}=6.8)$ & $0.81(3 \mathrm{H}, \mathrm{d}, \mathrm{J}=6.4)$ & $0.82(3 \mathrm{H}, \mathrm{d}, \mathrm{J}=6.8)$ \\
\hline 30 & $1.25(3 \mathrm{H}, \mathrm{d}, \mathrm{J}=6.8)$ & $1.34(3 \mathrm{H}, \mathrm{d}, \mathrm{J}=7.2)$ & $1.25(3 \mathrm{H}, \mathrm{d}, \mathrm{J}=6.8)$ & $1.34(3 \mathrm{H}, \mathrm{d}, \mathrm{J}=6.8)$ \\
\hline
\end{tabular}

